\title{
Space-Time Codes and Concatenated Channel Codes for Wireless Communications
}

\author{
T. H. LIEW AND LAJOS HANZO
}

\section{Contributed Paper}

Following a brief historical perspective on channel coding, an introduction to space-time block codes is given. The various space-time codes considered are then concatenated with a range of channel codecs, such as convolutional and block-based turbo codes as well as conventional and turbo trellis codes. The associated estimated complexity issues and memory requirements are also considered. These discussions are followed by a performance study of various space-time and channel-coded transceivers. Our aim is first to identify a space-time code/channel code combination constituting a good engineering tradeoff in terms of its effective throughput, bit-error-rate performance, and estimated complexity. Specifically, the issue of bit-to-symbol mapping is addressed in the context of convolutional codes (CCs) and convolutional coding as well as Bose-Chaudhuri-Hocquenghem coding-based turbo codes in conjunction with an attractive unity-rate space-time code and multilevel modulation is detailed. It is concluded that over the nondispersive or narrow-band fading channels, the best performance versus complexity tradeoff is constituted by Alamouti's twin-antenna block space-time code concatenated with turbo convolutional codes. Further comparisons with space-time trellis codes result in similar conclusions.

Keywords-Channel coding, concatenated coding, FEC, history of channel coding, space-time coding, STBC, STTC.

\section{INTRODUCTION}

The third-generation (3G) mobile communications standards [1] are expected to provide a wide range of user services, spanning from voice to high-rate data services, supporting rates of at least $144 \mathrm{~kb} / \mathrm{s}$ in vehicular, $384 \mathrm{~kb} / \mathrm{s}$ in outdoor-to-indoor, and $2 \mathrm{Mb} / \mathrm{s}$ in indoor as well as picocellular applications [1].

In an effort to support such high rates, the bit/symbol capacity of band-limited wireless channels can be increased by employing multiple antennas [2]. The classic approach is to use multiple antennas at the receiver and employ maximum ratio combining (MRC) [3]-[5] of the received signals for improving the performance. However, applying re-

Manuscript received March 11, 2001; revised October 15, 2001.

The authors are with the Department of Electronics and Computer Science, University of Southampton, Highfield, Southampton SO17 1BJ, U.K. (e-mail: 1h@ecs.soton.ac.uk).

Publisher Item Identifier S 0018-9219(02)01134-9. ceiver diversity at the mobile stations (MSs) increases their complexity. Hence, receiver diversity techniques typically have been applied at the base stations (BSs), although the Japanese personal handyphone system, know as PHS, employs second-order diversity, whereas the second receiver diversity antenna is invisible, since it is inside the handset (BS). In contrast, BSs provide services for many MSs and, hence, upgrading the BS's receivers in order to support antenna diversity is economically more viable. However, the drawback of this scheme is that it only provides diversity gain for the BSs' receivers.

In the past, different transmit diversity techniques have been introduced in order to provide diversity gain for MSs by upgrading the BSs. These transmit diversity techniques can be classified into three main categories: 1) schemes using information feedback [6], [7]; 2) arrangements invoking feedforward or training information [8]-[10]; and 3) blind schemes [11], [12]. Recently, Tarokh et al. proposed space-time trellis (STT) coding [13]-[19] by jointly designing the channel coding, modulation, transmit diversity, and the optional receiver diversity scheme. The performance criteria for designing STT codes were derived in [13] under the assumption that the channel is fading slowly and that the fading is frequency nonselective. These advances were then also extended to fast fading channels. The encoding and decoding complexity of these STT codes is comparable to that of conventional trellis codes [20]-[22] often employed in practice over nondispersive Gaussian channels.

STT codes [13]-[18] perform extremely well at the cost of relatively high complexity. In addressing the issue of decoding complexity, Alamouti [23] discovered a remarkable scheme for transmission using two transmit antennas. A simple decoding algorithm was also introduced by Alamouti [23], which can be generalized to an arbitrary number of receiver antennas. This scheme is significantly less complex than STT coding using two transmitter antennas, although there is a loss in performance [24]. Despite the associated performance penalty, Alamouti's scheme is appealing in terms of its simplicity and performance. This proposal 
motivated Tarokh et al. [24], [25] to generalize Alamouti's scheme to an arbitrary number of transmitter antennas, leading to the concept of space-time block (STB) codes.

Intrigued by the decoding simplicity of the STB codes proposed in [23]-[25], we commence our discourse in this paper by detailing their encoding and decoding process. Subsequently, we investigate the performance of the STB codes over perfectly interleaved nondispersive Rayleigh fading channels. A similar study of STT codes employed over wide-band correlated fading channels is provided in [26]. A system that consists of STB codes and different channel coders will be proposed. Finally, the performance and estimated complexity of the different systems will be compared and tabulated.

Following a brief historical perspective on channel coding in Section II, a rudimentary introduction to STB codes is given in Section IV and channel-coded space-time codes in Section V. The associated estimated complexity issues and memory requirements are addressed in Section V-C. The bulk of this contribution is constituted by the performance study of various space-time and channel-coded transceivers in Section VI. Our aim is first to identify a STB code/channel code combination constituting a good engineering tradeoff in terms of its effective throughput, bit-error-rate (BER) performance, and estimated complexity in Section VI-A. Specifically, the issue of bit-to-symbol mapping is addressed in the context of convolution codes and convolutional coding as well as Bose-Chaudhuri-Hocquenghem (BCH) codingbased turbo codes in conjunction with an attractive unity-rate space-time code and multilevel modulation in Section VI-B. These schemes are also benchmarked against a range of powerful trellis-coded modulation (TCM) and turbo trellis-coded modulation (TTCM) schemes. The merits of the various concatenated channel-coded and STB-coded schemes are highlighted in Section VI-D in the context of their coding gain versus estimated complexity tradeoffs. Our discussions are also extended to the comparison of channel-coded STB codes and STT codes in Section VI-E.

\section{Historical Perspective on Channel Coding}

The history of channel coding and forward error correction (FEC) coding dates back to Shannon's pioneering work [27] in 1948, predicting that arbitrarily reliable communications is achievable with the aid of channel coding, upon adding redundant information to the transmitted messages. However, Shannon refrained from proposing explicit channel-coding schemes for practical implementations. Furthermore, although upon increasing the amount of redundancy added the associated information delay increases, he did not specify the maximum delay that may have to be tolerated in order to be able to communicate near the Shannon limit. In recent years, researchers have been endeavoring to reduce the amount of latency inflicted, e.g., by a turbo codec's interleaver that has to be tolerated for the sake of attaining a given target performance.

Historically, one of the first practical FEC codes was the single error-correcting Hamming code [28], which was a block code proposed in 1950. Convolutional FEC codes that were discovered by Elias date back to 1955 [29]. Wozencraft and Reiffen [30], [31] as well as Fano [32] and Massey [33] have proposed various algorithms for their decoding. A major milestone in the history of convolutional error correction coding was the discovery of a maximum likelihood sequence estimation algorithm by Viterbi [34] in 1967. A classic interpretation of the Viterbi algorithm (VA) can be found, e.g., in Forney's often-quoted paper [35]. One of the first practical applications of convolutional codes (CCs) was proposed by Heller and Jacobs [36] during the seventies.

We note here that the VA does not result in minimum BER, it rather finds the most likely transmitted sequence of transmitted bits. However, it performs close to the minimum possible BER, which can be achieved only with the aid of the extremely complex full-search algorithm evaluating the probability of all possible $2^{n}$ binary strings of a $k$-bit message. The minimum BER decoding algorithm was proposed in 1974 by Bahl et al. [37], which was termed the maximum a posteriori (MAP) algorithm. Although the MAP algorithm slightly outperforms the VA in BER terms, because of its significantly higher complexity, it was rarely used in practice until turbo codes were contrived by Berrou et al. in 1993 [38], [39].

Focusing our attention on block codes, the single-error correcting Hamming block code was too weak for practical applications. An important practical milestone was the discovery of the family of multiple error correcting $\mathrm{BCH}$ binary block codes [40] in 1959 and in 1960 [41], [42]. In 1960, Peterson [43] recognized that these codes exhibit a cyclic structure, implying that all cyclically shifted versions of a legitimate codeword are also legitimate codewords. The first method for constructing trellises for linear block codes was proposed by Wolf [44] in 1978. Due to the associated high complexity, there was only limited research in trellis decoding of linear block codes [45], [46]. It was in 1988 when Forney [47] showed that some block codes have relatively simple trellis structures. Motivated by Forney's work, Honary, Markarian, and Farrell et al. [45], [48]-[51] as well as Lin and Kasami et al. [46], [52], [53] proposed various methods for reducing the associated decoder complexity. The Chase algorithm [54] is one of the most popular techniques used for near maximum likelihood decoding of block codes.

In 1961, Gorenstein and Zierler [55] extended binary coding theory to treat nonbinary codes as well, where code symbols constitute a number of bits, and this led to the birth of burst-error-correcting codes. They also contrived a combination of algorithms, which is referred to as the Peterson-Gorenstein-Zierler (PGZ) algorithm. In 1960, a prominent nonbinary subset of $\mathrm{BCH}$ codes was discovered by Reed and Solomon [56]; they were named Reed-Solomon (RS) codes after their inventors. These codes exhibit certain optimality properties, since their codewords have the highest possible minimum distance between the legitimate codewords for a given code rate. This, however, does not necessarily guarantee attaining the lowest possible BER. The PGZ decoder can also be invoked for decoding nonbinary RS codes. A range of powerful decoding algorithms for RS codes was found by Berlekamp [57], [58] and Massey 
[59], [60]. Various soft decision-decoding algorithms were proposed for soft decoding of RS codes by Oh and Sweeney [61], [62], Burgess et al. [63], and Honary [45]. In recent years, RS codes have found many practical applications, e.g., in compact disc players, in deep-space scenarios [64], and in the family of digital video broadcasting (DVB) schemes [65], which were standardized by the European Telecommunications Standardization Institute.

Inspired by the ancient theory of residue number systems (RNS) [66]-[68], which constitute a promising number system for supporting fast arithmetic operations [66], [67], a novel class of nonbinary codes referred to as redundant (RRNS) codes were introduced in 1967. An RRNS code is a maximum-minimum distance block code, exhibiting similar distance properties to RS codes. Watson and Hastings [68] as well as Krishna et al. [69], [70] exploited the properties of the RRNS for detecting or correcting a single error and also for detecting multiple errors. Recently, the soft decoding of RRNS codes was proposed in [71].

During the early 1970s, FEC codes were incorporated in various deep-space and satellite communications systems and, in the 1980s, they also became common in virtually all cellular mobile radio systems. However, for a long time, FEC codes and modulation have been treated as distinct subjects in communication systems. By integrating FEC and modulation, in 1987, Ungerboeck [20]-[22] proposed TCM, which is capable of achieving significant coding gains over power and band-limited transmission media. A further historic breakthrough was the invention of turbo codes by Berrou et al. [38], [39] in 1993, which facilitate the operation of communications systems near Shannon limits. Since its recent invention, turbo coding has evolved at an unprecedented rate and has reached a state of maturity within just a few years due to the intensive research efforts of the turbo coding community. As a result of this dramatic evolution, turbo coding has also found its way into standardized systems, such as, e.g., the recently ratified $3 \mathrm{G}$ mobile radio systems [72]. Even more impressive performance gains can be attained with the aid of turbo coding in the context of video broadcast systems, where the associated system delay is less critical, than in delay-sensitive interactive systems.

Turbo coding is based on a composite codec constituted of two parallel concatenated codecs. More specifically, in their proposed scheme, Berrou et al. [38], [39] used a parallel concatenation of two recursive systematic convolutional (RSC) codes, accommodating the turbo interleaver between the two encoders. At the decoder, an iterative structure using a modified version of the classic minimum BER MAP algorithm invented by Bahl et al. [37] was invoked by Berrou et al. in order to decode these parallel concatenated codes. Again, since 1993, a large body of work has been carried out in the area aiming, e.g., at reducing the associated decoder complexity. Practical reduced-complexity decoders are, e.g., the maximum logarithmic MAP (Max-Log-MAP) algorithm proposed by Koch and Baier [73], as well as by Erfanian $e t$ al. [74], the Log-MAP algorithm suggested by Robertson $e t$ al. [75], and the SOVA algorithm advocated by Hagenauer as well as Hoeher [76], [77]. Le Goff et al. [78], Wachsmann and Huber [79] as well as Robertson and Worz [80] suggested using these codes in conjunction with bandwidth efficient modulation schemes. Further advances in understanding the excellent performance of the codes are due, e.g., to Benedetto and Montorsi [81], [82] and Perez et al. [83]. During the mid-1990s, Hagenauer et al. [84] as well as Pyndiah [85] extended the turbo concept to parallel concatenated block codes as well. Nickl et al. [86] show that Shannon's limit can be approached within $0.27 \mathrm{~dB}$ by employing a simple turbo Hamming code. In [87], Acikel and Ryan proposed an efficient procedure for designing the puncturing patterns for high-rate turbo CCs. Jung and Nasshan [88], [89] characterized the achievable turbo-coded performance under the constraints of short transmission frame lengths, which is characteristic of interactive speech systems. In collaboration with Blanz, they also applied turbo codes to a CDMA system using joint detection and antenna diversity [90]. Barbulescu and Pietrobon addressed the issues of interleaver design [91]. The tutorial paper by Sklar [92] is also highly recommended as background reading.

Driven by the desire to support high data rates for a wide range of bearer services, Tarokh et al. [13] proposed STT codes in 1998. By jointly designing the FEC, modulation, transmit diversity, and optional receive diversity scheme, they increased the throughput of band-limited wireless channels. A few months later, Alamouti [23] invented a low-complexity STB code, which offers significantly lower complexity at the cost of a slight performance degradation. Alamouti's invention motivated Tarokh et al. [24], [25] to generalize Alamouti's scheme to an arbitrary number of transmitter antennas. Then, Bauch et al. [93], [94], Agrawal [95], Li et al. [96], [97], and Naguib et al. [98] extended the research of STB and STT codes from considering narrow-band channels to dispersive channels [13], [17], [23], [25], [98].

The evolution of channel-coding research over the past 50 years since Shannon's seminal contribution [27] is shown in Fig. 1. These milestones have also been incorporated in the range of monographs and textbooks summarized in Fig. 2. At the time of this writing, the Shannon limit has been approached for transmission over Gaussian channels within $0.27 \mathrm{~dB}$ [86]. Now the challenge is to contrive FEC schemes, which are capable of achieving a performance near the capacity of wireless channels. The design of an attractive channel-coding and modulation scheme depends on a range of competing factors, which are portrayed in Fig. 3. The message of this illustration is multifold. For example, given a certain transmission channel, it is always feasible to design a joint coding and modulation scheme, which can further reduce the BER achieved. This typically implies, however, further complexity and costs and coding/interleaving delay as well as reduced effective throughput. Different solutions accrue when optimizing different codec features. For example, in many applications, the most important codec parameters is the achievable coding gain, which quantifies the amount of bit-energy reduction at a certain target BER attained by a codec. Naturally, transmitted power reduction is extremely important in battery-powered devices. This 


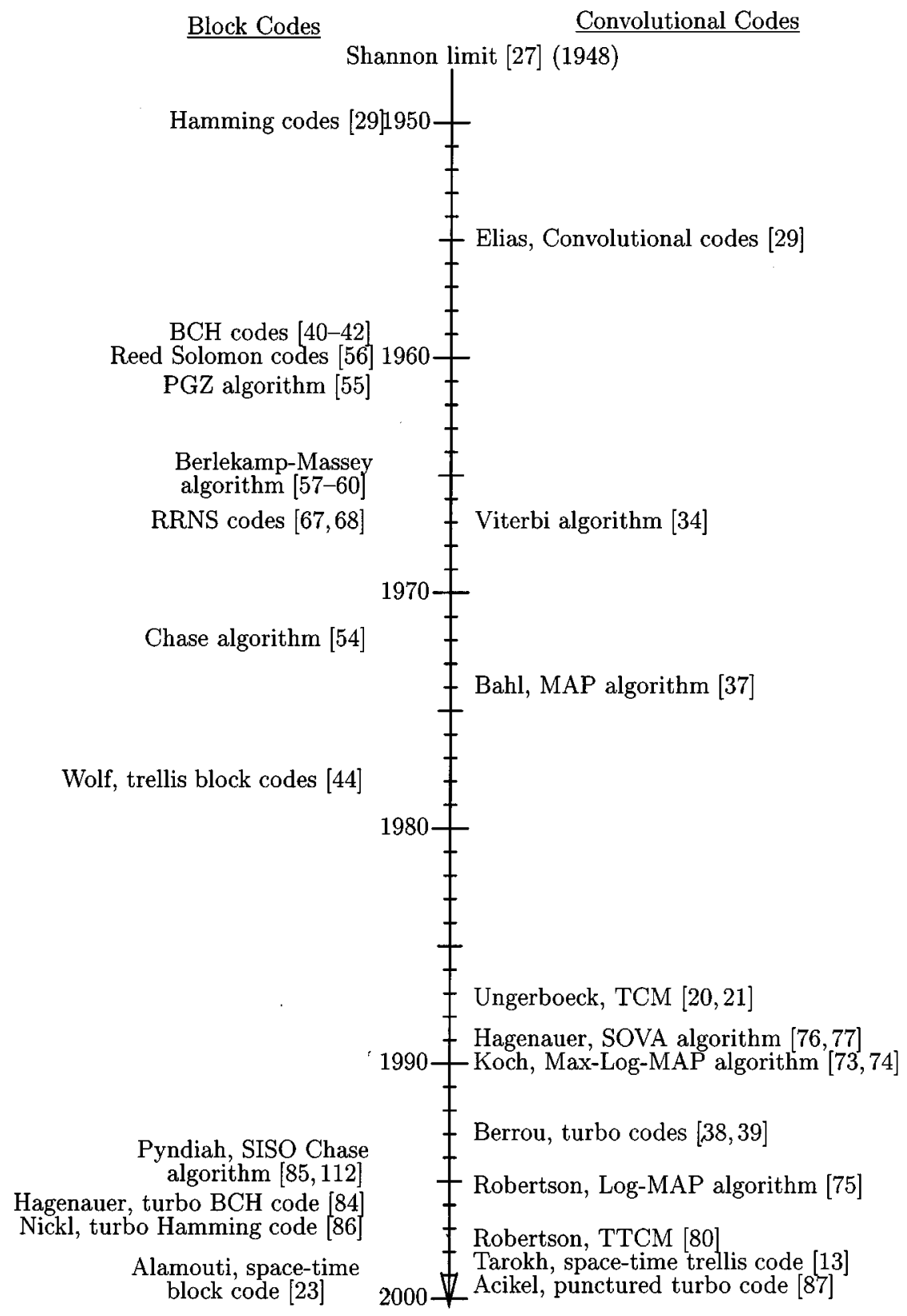

Fig. 1. Brief history of channel coding.

transmitter power reduction is only achievable at the cost of an increased implementational complexity, which itself typically increases the power consumption and, hence, erodes some of the power gain.

Viewing this system-optimization problem from a different perspective, it is feasible to transmit at a higher bit rate in a given fixed bandwidth by increasing the number of bits per modulated symbol. However, when aiming for a given target BER, the channel coding rate has to be reduced in order to increase the transmission integrity. Naturally, this reduces the effective throughput of the system and results in an overall increased system complexity. When the channel's characteristic and the associated bit-error statistics change, different solutions may become more attractive. This is because Gaussian channels, narrow-band and wide-band Rayleigh fading, or various Nakagami fading channels inflict different impairment. This paper examines these design tradeoffs and proposes a range of practical solutions. Following the above historical perspective on channel coding, in Section III, we will focus our attention on the family of space-time codes. The motivation of the forthcoming section is to portray space-time codes as a solution to creating attractive coding scheme for transmission over fading wireless rather than conventional Gaussian channels.

\section{Overview of Space-Time Codes}

In this section, we present a brief overview of STB codes by considering the classic MRC technique [23], [99], [100]. The introduction of this classic technique is important for understanding the construction of STB codes. 


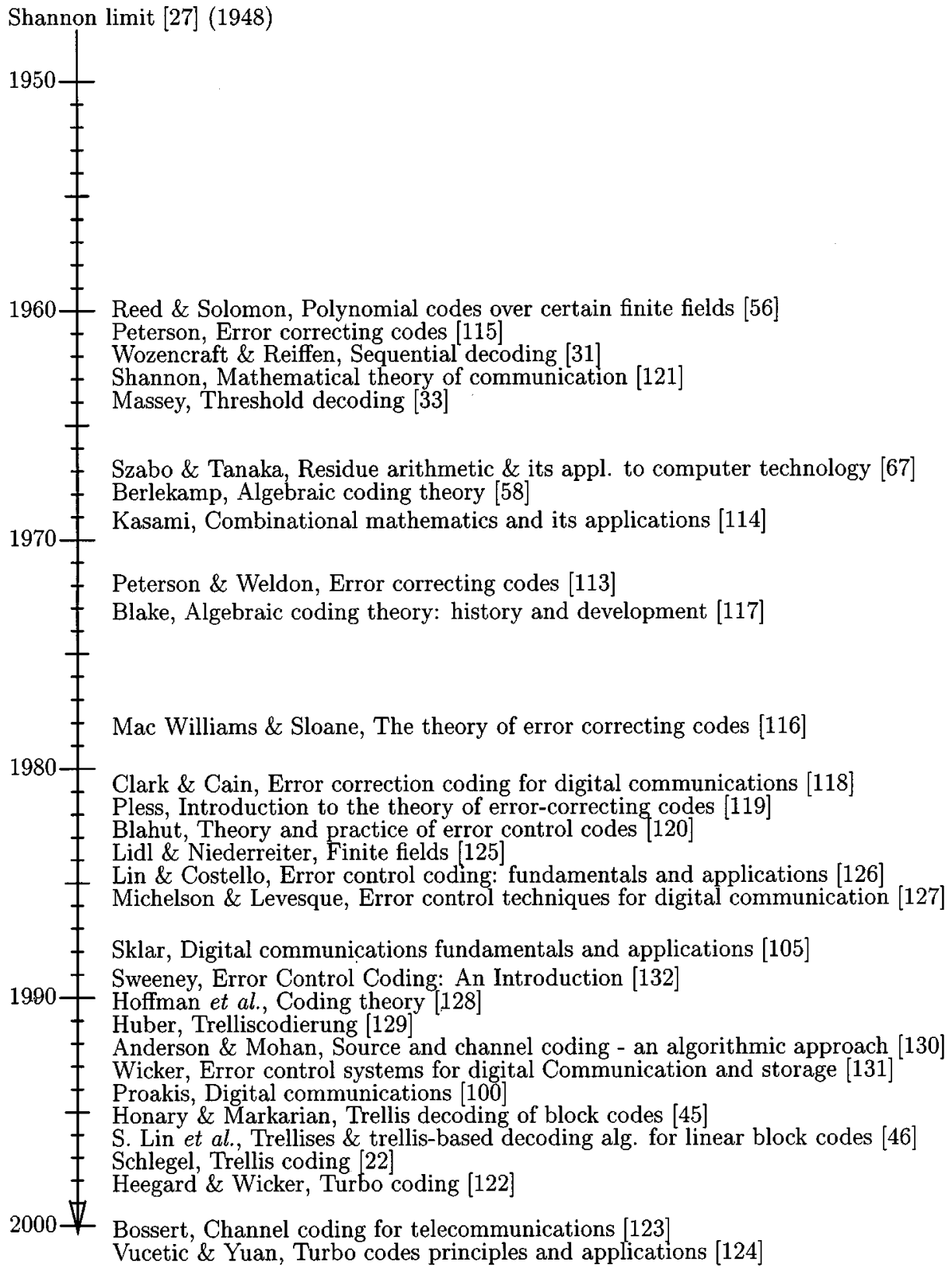

Fig. 2. Milestones in channel coding.

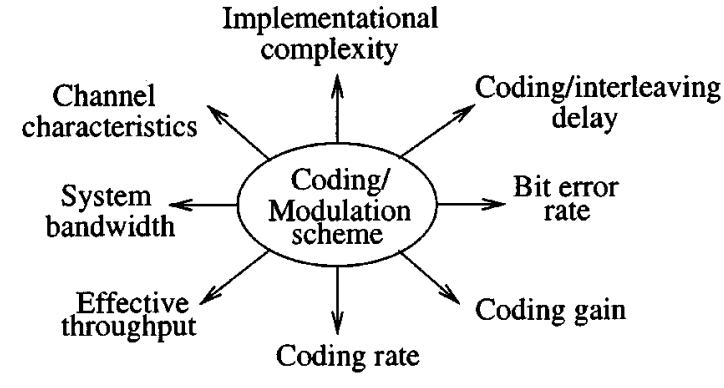

Fig. 3. Factors affecting the design of channel coding and modulation schemes.

\section{A. Maximum Ratio Combining}

In conventional transmission systems, we have a single transmitter, which transmits information to a single receiver.
In Rayleigh fading channels, the transmitted symbols experience severe magnitude fluctuation and phase rotation. In order to mitigate this problem, we can employ several receivers that receive replicas of the same transmitted symbol through independent fading paths. Even if a particular path is severely faded, we may still be able to recover a reliable estimate of the transmitted symbols through other propagation paths. However, at the station, we have to combine the received symbols of the different propagation paths, which involves additional complexity. An optimal combining method often used in practice is referred to as the MRC technique [23], [99], [100].

Fig. 4 shows the baseband representation of the classic MRC technique in conjunction with two receivers. At a particular instant, a symbol $x$ is transmitted. As we can see from the figure, the transmitted symbol $x$ propagates through 


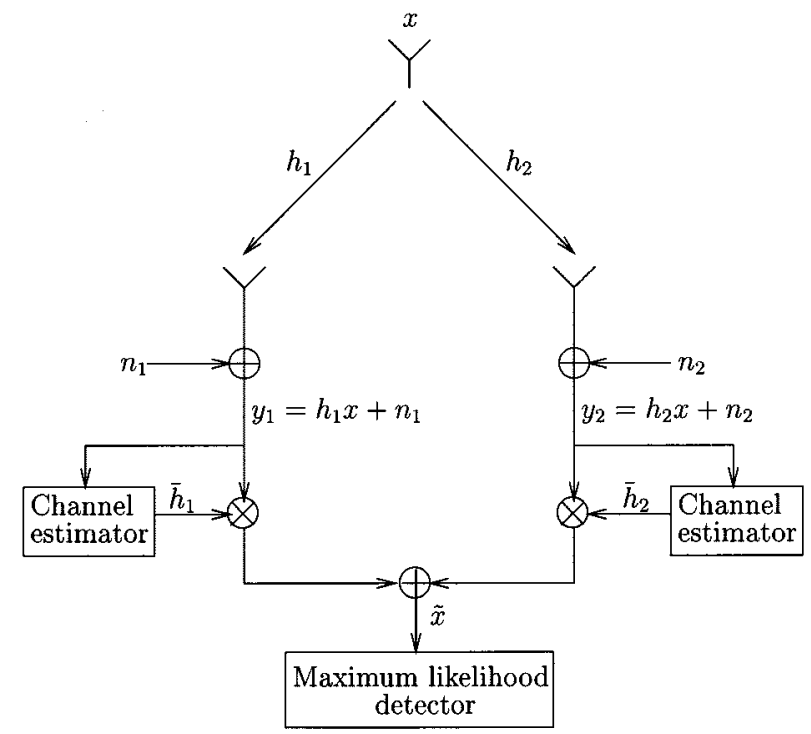

Fig. 4. Baseband representation of the MRC technique using two receivers.

two different channels, namely, $h_{1}$ and $h_{2}$. For simplicity, all channels are assumed to be constituted of a single nondispersive or flat-fading propagation path and can be modeled as complex multiplicative distortion, which consists of a magnitude and phase response given by

$$
\begin{aligned}
& h_{1}=\left|h_{1}\right| e^{j \theta_{1}} \\
& h_{2}=\left|h_{2}\right| e^{j \theta_{2}}
\end{aligned}
$$

where $\left|h_{1}\right|,\left|h_{2}\right|$ are the fading magnitudes and $\theta_{1}, \theta_{2}$ are the phase values. Noise is unavoidably added by each receiver, as shown in Fig. 4. Hence, the resulting received baseband signals are

$$
\begin{aligned}
& y_{1}=h_{1} x+n_{1} \\
& y_{2}=h_{2} x+n_{2}
\end{aligned}
$$

where $n_{1}$ and $n_{2}$ are complex noise samples. In matrix form, this can be written as

$$
\left(\begin{array}{l}
y_{1} \\
y_{2}
\end{array}\right)=x\left(\begin{array}{l}
h_{1} \\
h_{2}
\end{array}\right)+\left(\begin{array}{l}
n_{1} \\
n_{2}
\end{array}\right)
$$

Assuming that perfect channel information is available, the received signals $y_{1}$ and $y_{2}$ can be multiplied by the conjugate of the complex channel transfer functions $\bar{h}_{1}$ and $\bar{h}_{2}$, respectively, in order to remove the channel's effects. Then, the corresponding signals are combined at the input of the maximum likelihood detector of Fig. 4 according to

$$
\begin{aligned}
\tilde{x} & =\bar{h}_{1} y_{1}+\bar{h}_{2} y_{2} \\
& =\bar{h}_{1} h_{1} x+\bar{h}_{1} n_{1}+\bar{h}_{2} h_{2} x+\bar{h}_{2} n_{2} \\
& =\left(\left|h_{1}\right|^{2}+\left|h_{2}\right|^{2}\right) x+\bar{h}_{1} n_{1}+\bar{h}_{2} n_{2} .
\end{aligned}
$$

The combined signal $\tilde{x}$ is then passed to the maximum likelihood detector, as shown in Fig. 4. The most likely transmitted symbol is determined by the maximum likelihood detector based on the Euclidean distances between the com- bined signal $\tilde{x}$ and all possible transmitted symbols. The simplified decision rule is based on choosing $x_{i}$ if and only if

$$
\operatorname{dist}\left(\tilde{x}, x_{i}\right) \leq \operatorname{dist}\left(\tilde{x}, x_{j}\right), \quad \forall i \neq j
$$

where $\operatorname{dist}(A, B)$ is the Euclidean distance between signals $A$ and $B$ and the index $j$ spans all possible transmitted signals. From (7), we can see that maximum likelihood transmitted symbol is the one having the minimum Euclidean distance from the combined signal $\tilde{x}$.

\section{SPACE-Time BLock Codes}

In Section III, we have introduced briefly the classic MRC technique. In this section we will present the basic principles of STB codes following the seminal contributions by Tarokh et al. [23]-[25]. In analogy to the MRC matrix formula of (5), a STB code describing the relationship between the original transmitted signal $x$ and the signal replicas artificially created at the transmitter for transmission over various diversity channels is defined by an $n \times p$ dimensional transmission matrix. The entries of the matrix are constituted of linear combinations of the $k$-ary input symbols $x_{1}, x_{2}, \ldots, x_{k}$ and their conjugates. The $k$-ary input symbols $x_{i} i=1 \cdots k$ are used to represent the information-bearing binary bits to be transmitted over the transmit diversity channels. In a signal constellation having $2^{b}$ constellation points, a number $b$ of binary bits are used to represent a symbol $x_{i}$. Hence, a block of $k \times b$ binary bits are entered into the STB encoder at a time and it is, therefore, referred to as a STB code. The number of transmitter antennas is $p$ and $n$ represents the number of time slots used to transmit $k$ input symbols. Hence, a general form of the transmission matrix of a STB code is written as

$$
\left(\begin{array}{cccc}
g_{11} & g_{21} & \cdots & g_{p 1} \\
g_{12} & g_{22} & \cdots & g_{p 2} \\
\cdot & \cdot & \cdot & \cdot \\
\cdot & \cdot & \cdot & \cdot \\
g_{1 n} & g_{2 n} & \cdots & g_{p n}
\end{array}\right)
$$

where the entries $g_{i j}$ represent linear combinations of the symbols $x_{1}, x_{2}, \ldots, x_{k}$ and their conjugates. More specifically, the entries $g_{i j}$, where $i=1 \cdots p$ are transmitted simultaneously from transmit antennas $1, \ldots, p$ in each time slot $j=1, \ldots, n$. For example, in time slot $j=2$, signals $g_{12}, g_{22}, \ldots, g_{p 2}$ are transmitted simultaneously from transmit antennas $T x 1, T x 2, \ldots, T x p$. We can see in the transmission matrix defined in (8) that encoding is carried out in both space and time; hence, the term space-time coding.

The $n \times p$ transmission matrix in (8) (which defines the STB code) is based on a complex generalized orthogonal design, as defined in [23]-[25]. Since there are $k$ symbols transmitted over $n$ time slots, the code rate of the STB code is given by

$$
R=k / n \text {. }
$$

At the receiving end, one can have an arbitrary number of $q$ receivers. It was shown in [23] that the associated diversity order is $p \times q$. A combining technique [23]-[25] similar to MRC can be applied at the receiving end, which 
Table 1

Encoding and Transmission Process for the $\mathbf{G}_{2}$ STB Code of (10)

\begin{tabular}{c|c|c}
\hline \multirow{2}{*}{ Time } & \multicolumn{2}{|c}{ antenna } \\
\cline { 2 - 3 } slot, $T$ & $T x 1$ & $T x 2$ \\
\hline 1 & $x_{1}$ & $x_{2}$ \\
\hline 2 & $-\bar{x}_{2}$ & $\bar{x}_{1}$ \\
\hline
\end{tabular}

may be generalized to $q$ receivers. Current state-of-the-art designs assume the associated diversity channels to be flat fading channels. A possible approach to satisfying this condition for high-rate transmissions over frequency-selective channels is to split the high-rate bit stream into a large number of low-rate streams transmitted over flat-fading subchannels. This can be achieved with the aid of orthogonal frequency division multiplexing (OFDM) [101]. Then, the complex fading envelope may be considered constant over $n$ consecutive time slots.

\section{A. Twin-Transmitter-Based STB Code}

As mentioned above, the simplest form of STB codes, which is a simple twin-transmitter-based scheme associated with $p=2$, was proposed by Alamouti in [23]. The transmission matrix is

$$
\mathrm{G}_{2}=\left(\begin{array}{rr}
x_{1} & x_{2} \\
-\bar{x}_{2} & \bar{x}_{1}
\end{array}\right)
$$

We can see in the transmission matrix $\mathbf{G}_{2}$ that there are $p=2$ (number of columns in the matrix $\mathbf{G}_{2}$ ) transmitters, $k=2$ possible input symbols, namely, $x_{1} x_{2}$, and the code spans over $n=2$ (number of rows in the matrix $\mathbf{G}_{2}$ ) time slots. Since $k=2$ and $n=2$, the code rate given by (9) is unity. The associated encoding and transmission process is shown in Table 1.

At any given time instant $T$, two signals are simultaneously transmitted from the antennas $T x 1$ and $T x 2$. For example, in the first time slot associated with $T=1$, signal $x_{1}$ is transmitted from antenna $T x 1$ and simultaneously signal $x_{2}$ is transmitted from antenna $T x 2$. In the next time slot corresponding to $T=2$, signals $-\bar{x}_{2}$ and $\bar{x}_{1}$ (the conjugates of symbols $x_{1}$ and $x_{2}$ ) are simultaneously transmitted from antennas $T x 1$ and $T x 2$, respectively.

1) Space-Time Code $\mathbf{G}_{2}$ Using One Receiver: Let us now consider an example of encoding and decoding the $\mathbf{G}_{2}$ STB code of (10) using one receiver. This example can be readily extended to an arbitrary number of receivers. In Fig. 5, we show the baseband representation of a simple two-transmitter STB code, namely, that of the $\mathbf{G}_{2}$ code seen in (10) using one receiver. We can see from the figure that there are two transmitters, namely, $T x 1$ as well as $T x 2$ and they transmit two signals simultaneously. As mentioned earlier, the complex fading envelope is assumed to be constant across the corresponding two consecutive time slots. Therefore, one can write

$$
\begin{aligned}
& h_{1}=h_{1}(T=1)=h_{1}(T=2) \\
& h_{2}=h_{2}(T=1)=h_{2}(T=2) .
\end{aligned}
$$

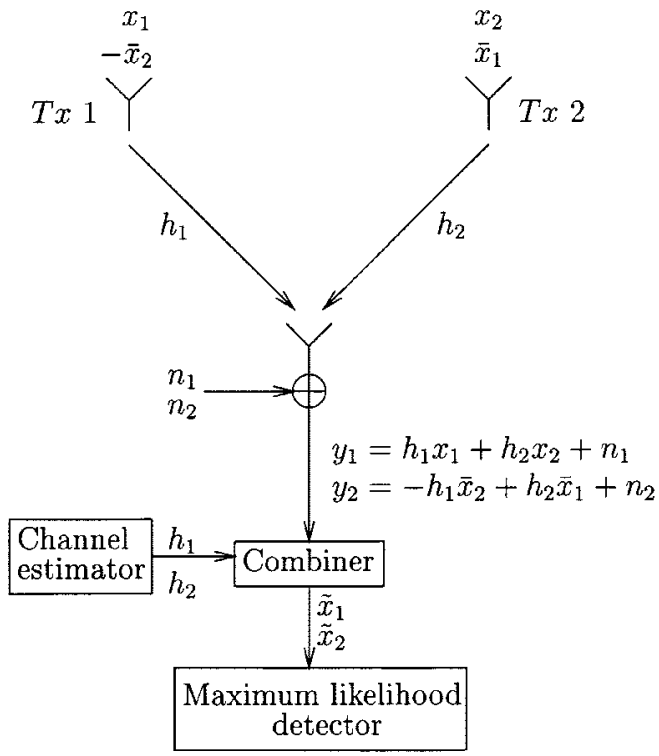

Fig. 5. Baseband representation of the simple twin-transmitter STB code $\mathbf{G}_{2}$ of (10) using one receiver.

Independent noise samples are added by the receiver in each time slot and hence the signals received over nondispersive or narrow-band channels can be expressed with the aid of (10) as

$$
\begin{aligned}
& y_{1}=h_{1} x_{1}+h_{2} x_{2}+n_{1} \\
& y_{2}=-h_{1} \bar{x}_{2}+h_{2} \bar{x}_{1}+n_{2}
\end{aligned}
$$

where $y_{1}$ is the first received signal and $y_{2}$ is the second. Note that the received signal $y_{1}$ consists of the transmitted signals $x_{1}$ and $x_{2}$, while $y_{2}$ consists of their conjugates. In order to determine the transmitted symbols, we have to extract the signals $x_{1}$ and $x_{2}$ from the received signals $y_{1}$ and $y_{2}$. Therefore, both signals $y_{1}$ and $y_{2}$ are passed to the combiner, as shown in Fig. 5. In the combiner-aided by the channel estimator, which provides perfect estimation of the diversity channels in this example-simple signal processing is performed in order to separate the signals $x_{1}$ and $x_{2}$. Specifically, in order to extract the signal $x_{1}$, the received signals $y_{1}$ and $y_{2}$ are combined according to

$$
\begin{aligned}
\tilde{x}_{1} & =\bar{h}_{1} y_{1}+h_{2} \bar{y}_{2} \\
& =\bar{h}_{1} h_{1} x_{1}+\bar{h}_{1} h_{2} x_{2}+\bar{h}_{1} n_{1}-h_{2} \bar{h}_{1} x_{2}+h_{2} \bar{h}_{2} x_{1}+h_{2} \bar{n}_{2} \\
& =\left(\left|h_{1}\right|^{2}+\left|h_{2}\right|^{2}\right) x_{1}+\bar{h}_{1} n_{1}+h_{2} \bar{n}_{2} .
\end{aligned}
$$

Similarly, for signal $x_{2}$, we generate

$$
\begin{aligned}
\tilde{x}_{2} & =\bar{h}_{2} y_{1}-h_{1} \bar{y}_{2} \\
& =\bar{h}_{2} h_{1} x_{1}+\bar{h}_{2} h_{2} x_{2}+\bar{h}_{2} n_{1}+h_{1} \bar{h}_{1} x_{2}-h_{1} \bar{h}_{2} x_{1}-h_{1} \bar{n}_{2} \\
& =\left(\left|h_{1}\right|^{2}+\left|h_{2}\right|^{2}\right) x_{2}+\bar{h}_{2} n_{1}-h_{1} \bar{n}_{2} .
\end{aligned}
$$

Clearly, from (15) and (16), we can see that we have separated the signals $x_{1}$ and $x_{2}$ by simple multiplications and additions. Due to the orthogonality of the STB code $\mathbf{G}_{2}$ in (10) [24], the unwanted signal $x_{2}$ is canceled out in (15) and vice versa, signal $x_{1}$ is removed from (16). Both signals $\tilde{x}_{1}$ 


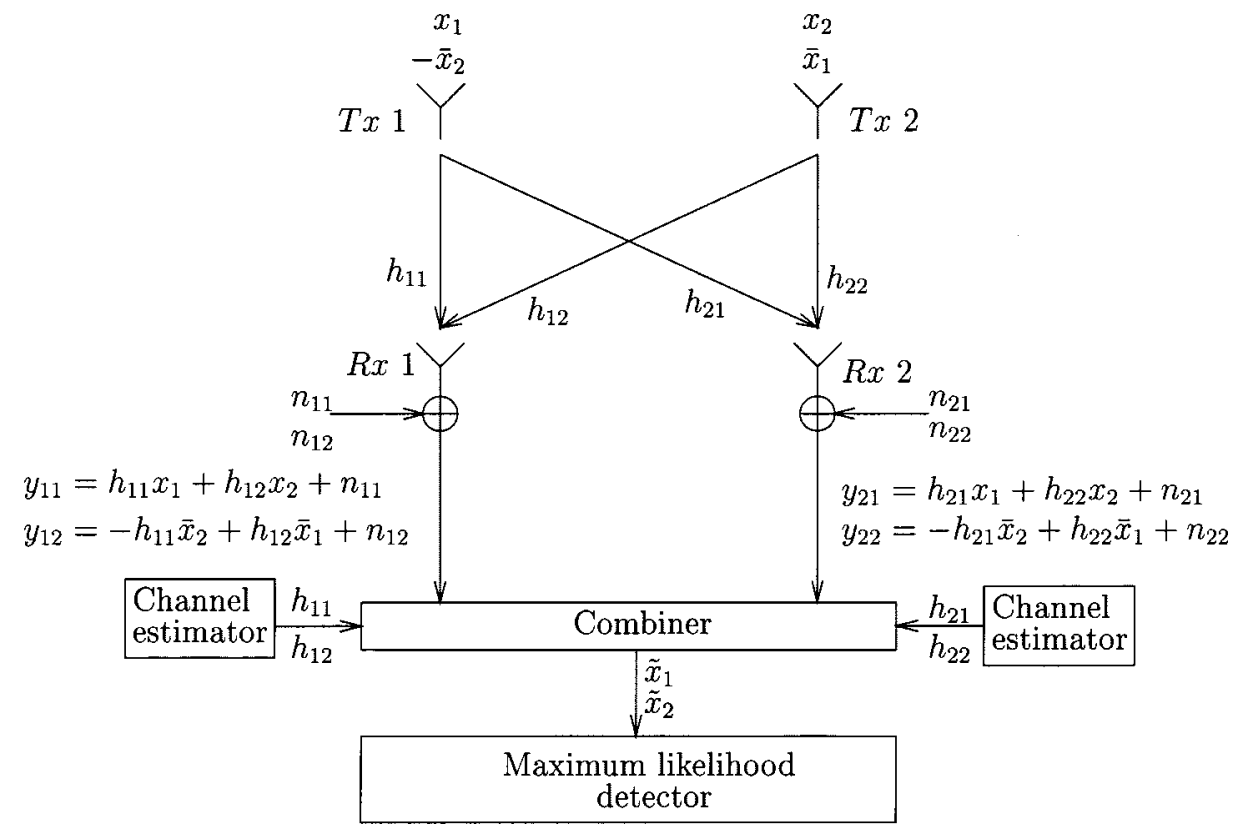

Fig. 6. Baseband representation of the simple twin-transmitter STB code $\mathbf{G}_{2}$ of (10) using two receivers.

and $\tilde{x}_{2}$ are then passed to the maximum likelihood detector of Fig. 5, which applies (7) to determine the most likely transmitted symbols.

From (15) and (16), we can derive a simple rule of thumb for manipulating the received signal in order to extract a symbol $x_{i}$. For each received signal $y_{j}$, we would have a linear combination of the transmitted signals $x_{i}$ convolved with the corresponding channel impulse response (CIR) $h_{i}$. The nondispersive CIR is assumed to be constituted by a single CIR tap corresponding to a complex multiplicative factor. The conjugate of the CIR $\bar{h}_{i}$ should be multiplied with the received signal $y_{j}$, if $x_{i}$ is in the expression of the received signal $y_{j}$. However, if the conjugate of $x_{i}$, namely, $\bar{x}_{i}$ is present in the expression, we should then multiply the CIR $h_{i}$ with the conjugate of the received signal $y_{j}$, namely, $\bar{y}_{j}$. The product should then be added to or subtracted from the intermediate result, depending on the sign of the term in the expression of the received signal $y_{j}$.

2) Space-Time Code $\mathbf{G}_{2}$ Using Two Receivers: In Section IV-A1, we have shown an example of the encoding and decoding process for the $\mathbf{G}_{2}$ STB code of (10) using one receiver. However, this example can be readily extended to an arbitrary number of receivers. The encoding and transmission sequence will be identical to the case of a single receiver. For illustration, we discuss the specific case of two transmitters and two receivers, as shown in Fig. 6. We will show, however, that the generalization to $q$ receivers is straightforward. In Fig. 6, the subscript $i$ in the notation $h_{i j}, n_{i j}$, and $y_{i j}$ represents the receiver index. By contrast, the subscript $j$ denotes the transmitter index in the CIR $h_{i j}$, but it denotes the time slot $T$ in $n_{i j}$ and $y_{i j}$. Therefore, at the first receiver $R x 1$, we have

$$
\begin{aligned}
& y_{11}=h_{11} x_{1}+h_{12} x_{2}+n_{11} \\
& y_{12}=-h_{11} \bar{x}_{2}+h_{12} \bar{x}_{1}+n_{12}
\end{aligned}
$$

while at receiver $R x 2$, we have

$$
\begin{aligned}
& y_{21}=h_{21} x_{1}+h_{22} x_{2}+n_{21} \\
& y_{22}=-h_{21} \bar{x}_{2}+h_{22} \bar{x}_{1}+n_{22}
\end{aligned}
$$

We can, however, generalize these equations to

$$
\begin{aligned}
& y_{i 1}=h_{i 1} x_{1}+h_{i 2} x_{2}+n_{i 1} \\
& y_{i 2}=-h_{i 1} \bar{x}_{2}+h_{i 2} \bar{x}_{1}+n_{i 2}
\end{aligned}
$$

where $i=1, \ldots, q$ and $q$ is the number of receivers, which is equal to two in this example. At the combiner of Fig. 6, the received signals are combined to extract the transmitted signals $x_{1}$ and $x_{2}$ from the received signals $y_{11}, y_{12}, y_{21}$, and $y_{22}$ according to

$$
\begin{aligned}
& \tilde{x}_{1}=\bar{h}_{11} y_{11}+h_{12} \bar{y}_{12}+\bar{h}_{21} y_{21}+h_{22} \bar{y}_{22} \\
& \tilde{x}_{2}=\bar{h}_{12} y_{11}-h_{11} \bar{y}_{12}+\bar{h}_{22} y_{21}-h_{21} \bar{y}_{22}
\end{aligned}
$$

Again, we can generalize the above expressions to $q$ receivers, yielding

$$
\begin{aligned}
& \tilde{x}_{1}=\sum_{i=1}^{q}\left(\bar{h}_{i 1} y_{i 1}+h_{i 2} \bar{y}_{i 2}\right) \\
& \tilde{x}_{2}=\sum_{i=1}^{q}\left(\bar{h}_{i 2} y_{i 1}-h_{i 1} \bar{y}_{i 2}\right) .
\end{aligned}
$$

Finally, we can simplify (23) and (24) to

$$
\begin{aligned}
\tilde{x}_{1}= & \left(\left|h_{11}\right|^{2}+\left|h_{12}\right|^{2}+\left|h_{21}\right|^{2}+\left|h_{22}\right|^{2}\right) \\
& \cdot x_{1}+\bar{h}_{11} n_{11}+h_{12} \bar{n}_{12}+\bar{h}_{21} n_{21}+h_{22} \bar{n}_{22} \\
\tilde{x}_{2}= & \left(\left|h_{11}\right|^{2}+\left|h_{12}\right|^{2}+\left|h_{21}\right|^{2}+\left|h_{22}\right|^{2}\right) \\
& \cdot x_{2}+\bar{h}_{12} n_{11}-h_{11} \bar{n}_{12}+\bar{h}_{22} n_{21}-h_{21} \bar{n}_{22}
\end{aligned}
$$


In the generalized form of $q$ receivers, we have

$$
\begin{aligned}
& \tilde{x}_{1}=\sum_{i=1}^{q}\left[\left(\left|h_{i 1}\right|^{2}+\left|h_{i 2}\right|^{2}\right) x_{1}+\bar{h}_{i 1} n_{i 1}+h_{i 2} \bar{n}_{i 2}\right] \\
& \tilde{x}_{2}=\sum_{i=1}^{q}\left[\left(\left|h_{i 1}\right|^{2}+\left|h_{i 2}\right|^{2}\right) x_{2}+\bar{h}_{i 2} n_{i 1}-h_{i 1} \bar{n}_{i 2}\right] .
\end{aligned}
$$

Signals $\widetilde{x}_{1}$ and $\widetilde{x}_{2}$ are finally derived and passed to the maximum likelihood detector seen in Fig. 6. Again, (7) is applied to determine the maximum likelihood transmitted symbols.

We observe in (29) that signal $x_{1}$ is multiplied by a term related to the fading amplitudes, namely, $\left|h_{i 1}\right|^{2}+\left|h_{i 2}\right|^{2}$. Hence, in order to acquire a high-reliability signal $\widetilde{x}_{1}$, the amplitudes of the CIRs must be large. If the number of receivers is equal to one, i.e., $q=1$, then (29) is simplified to (15). In (15), we can see that there are two fading amplitude terms, i.e., two independent paths associated with transmitting the symbol $x_{1}$. Therefore, if either of the paths is in a deep fade, the other path may still may provide a high reliability for the transmitted signal $x_{1}$. This explains why the performance of a system having two transmitters and one receiver is better than that of a system employing one transmitter and one receiver. On the other hand, in the conventional single-transmitter single-receiver system, there is only a single propagation path, which may be severely attenuated by a deep fade. To elaborate further, if the number of receivers is increased to $q=2$, (27) results from (29). We can see in (27) that there are now twice as many propagation paths, as in (15). This increases the probability of providing a high reliability for signal $\tilde{x}_{1}$.

\section{B. Other STB Codes}

In Section IV-A, we have detailed Alamouti's simple twotransmitter STB code, namely, the $\mathbf{G}_{2}$ code of (10). This code is significantly less complex than the STT codes of [13]-[18], which use two transmit antennas. However, again, there is a performance loss compared to the STT codes of [13]-[18]. Despite its performance loss, Alamouti's scheme [23] is appealing in terms of its simplicity. This motivated Tarokh et al. [24] to search for similar schemes using more than two transmit antennas. In [24], the theory of orthogonal code design was invoked in order to construct STB codes having more than two transmitters. The half-rate STB code employing three transmitters was defined [24]

$$
\mathbf{G}_{3}=\left(\begin{array}{rrr}
x_{1} & x_{2} & x_{3} \\
-x_{2} & x_{1} & -x_{4} \\
-x_{3} & x_{4} & x_{1} \\
-x_{4} & -x_{3} & x_{2} \\
\bar{x}_{1} & \bar{x}_{2} & \bar{x}_{3} \\
-\bar{x}_{2} & \bar{x}_{1} & -\bar{x}_{4} \\
-\bar{x}_{3} & \bar{x}_{4} & \bar{x}_{1} \\
-\bar{x}_{4} & -\bar{x}_{3} & \bar{x}_{2}
\end{array}\right)
$$

Table 2

Different STB Codes

\begin{tabular}{c|c|c|c|c}
\hline $\begin{array}{c}\text { Space-time } \\
\text { code }\end{array}$ & Rate & $\begin{array}{c}\text { No. of } \\
\text { transmitters, } p\end{array}$ & $\begin{array}{c}\text { No. of input } \\
\text { symbols, } k\end{array}$ & $\begin{array}{c}\text { Code } \\
\text { span, } n\end{array}$ \\
\hline $\mathbf{G}_{2}$ & $\mathbf{1}$ & 2 & 2 & 2 \\
\hline $\mathbf{G}_{3}$ & $1 / 2$ & 3 & 4 & 8 \\
\hline $\mathbf{G}_{4}$ & $1 / 2$ & 4 & 4 & 8 \\
\hline $\mathbf{H}_{3}$ & $\mathbf{3 / 4}$ & 3 & 3 & 4 \\
\hline $\mathbf{H}_{4}$ & $\mathbf{3 / 4}$ & 4 & 3 & 4 \\
\hline
\end{tabular}

and the four-transmitter half-rate STB code was specified [24]

$$
\mathbf{G}_{4}=\left(\begin{array}{rrrr}
x_{1} & x_{2} & x_{3} & x_{4} \\
-x_{2} & x_{1} & -x_{4} & x_{3} \\
-x_{3} & x_{4} & x_{1} & -x_{2} \\
-x_{4} & -x_{3} & x_{2} & x_{1} \\
\bar{x}_{1} & \bar{x}_{2} & \bar{x}_{3} & \bar{x}_{4} \\
-\bar{x}_{2} & \bar{x}_{1} & -\bar{x}_{4} & \bar{x}_{3} \\
-\bar{x}_{3} & \bar{x}_{4} & \bar{x}_{1} & -\bar{x}_{2} \\
-\bar{x}_{4} & -\bar{x}_{3} & \bar{x}_{2} & \bar{x}_{1}
\end{array}\right) .
$$

By employing the STB codes $\mathbf{G}_{3}$ and $\mathbf{G}_{4}$, we can see that the bandwidth efficiency has been reduced by a factor of two compared to the STB code $\mathbf{G}_{2}$. Furthermore, the number of transmission slots across that the channels is required to have a constant fading envelope is eight, namely, four times higher than that of the space-time code $\mathbf{G}_{2}$.

In order to increase the associated bandwidth efficiency, Tarokh et al. constructed the three-quarter rate so-called generalized complex orthogonal sporadic codes [24], [25]. The corresponding three-quarter rate three-transmitter STB code is given by [24]

$$
\mathbf{H}_{3}=\left(\begin{array}{ccc}
x_{1} & x_{2} & \frac{x_{3}}{\sqrt{2}} \\
-\bar{x}_{2} & \bar{x}_{1} & \frac{x_{3}}{\sqrt{2}} \\
\frac{\bar{x}_{3}}{\sqrt{2}} & \frac{\bar{x}_{3}}{\sqrt{2}} & \frac{\left(-x_{1}-\bar{x}_{1}+x_{2}-\bar{x}_{2}\right)}{2} \\
\frac{\bar{x}_{3}}{\sqrt{2}} & -\frac{\bar{x}_{3}}{\sqrt{2}} & \frac{\left(x_{2}+\bar{x}_{2}+x_{1}-\bar{x}_{1}\right)}{2}
\end{array}\right)
$$

while the three-quarter rate four-transmitter STB code is defined as shown in (34) at the bottom of the next page [24].

In Table 2, we summarize the parameters associated with all STB codes proposed by Alamouti [23] as well as Tarokh et al. [24], [25]. The decoding algorithms and the corresponding performances of the STB codes were given in [25].

\section{Channel-Coded Space-Time Block Codes}

In Section IV, we have given a detailed illustration of the concept of STB codes. Recently, Bauch [102] derived a simple symbol-by-symbol MAP decoding rule for STB codes. The soft-outputs provided by the space-time MAP decoder can be used as the input to channel decoders such as, e.g., turbo codes, which may be concatenated for further improving the system's performance. Accordingly, in this 


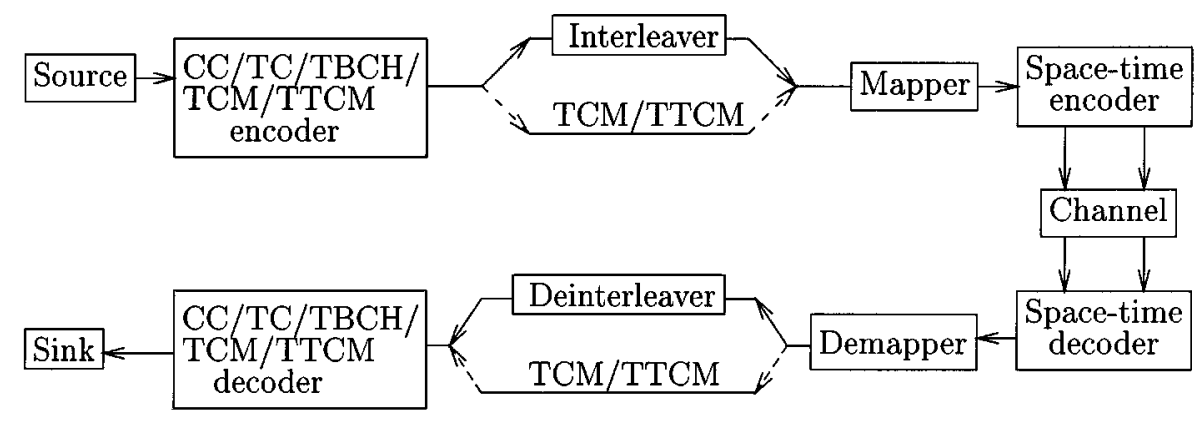

Fig. 7. System overview of STB codes and different channel coding schemes.

section, we concatenate STB codes with CCs [29], [103], [104], turbo convolutional (TC) codes [38], [39], turbo BCH (TBCH) codes [84], TCM [20], [21], and TTCM [80]. The performances and estimated complexities of the schemes will be studied and compared. We will also address the issue of mapping channel-coded bits of the TC and TBCH schemes to different protection classes in multilevel modulations [101].

CCs were first suggested by Elias [29] in 1955. The VA was proposed by Viterbi [34], [35] in 1967 for the maximum likelihood decoding of CCs. As an alternative decoder, the more complex MAP algorithm, which provides the optimum BER performance, was proposed by Bahl [37], although this was not significantly better than that of the VA. In the early 1970s, CCs were used in deep-space and satellite communications. They were then also adopted by the Global System of Mobile Communications (GSM) [72] for the pan-European digital cellular mobile radio system.

In 1993, Berrou et al. [38], [39] proposed a novel channel code, referred to as a turbo code. The turbo encoder consists of two component encoders. Generally, CCs are used as the component encoders and the corresponding turbo codes are termed here as a TC code. However, $\mathrm{BCH}$ [72], [105] codes can also be employed as their component codes, resulting in the TBCH codes. They have been shown, e.g., by Hagenauer et al. [84] and Nikl et al. [86] to perform impressively at nearunity coding rates, although at a higher decoding complexity than that of the corresponding-rate TCs.

In 1987, Ungerboeck [20], [21] invented TCM by combining the design of channel coding and modulation. TCM optimizes the Euclidean distance between codewords and hence maximizes the coding gain. In [80], Robertson et al. applied the basic idea of turbo codes [38], [39] to TCM by retaining the important properties and advantages of both structures. In the resultant TTCM scheme, two Ungerboeck codes [20], [21] are employed in combination with TCM as component codes in an overall structure similar to that of turbo codes.

\section{A. System Overview}

A schematic of the proposed concatenated STB codes and the different channel coding schemes is shown in Fig. 7. As mentioned above, the investigated channel coding schemes are CC, TC codes, TBCH codes, TCM, and TTCM. The information source in the transmitter of Fig. 7 generates random data bits. The information bits are then encoded by each of the above five different channel coding schemes. However, as seen in Fig. 7 only the output binary bits of the $\mathrm{CC}$, TBCH, and TC coding schemes are channel interleaved. The role of the interleaver will be detailed in Section VI-B.

The output bits of the TCM and TTCM scheme are passed directly to the mapper in Fig. 7, which employs two different mapping techniques. Gray mapping [78], [105] is used for the $\mathrm{CC}, \mathrm{TBCH}$, and TC schemes, whereas set-partitioning [20]-[22], [80] is utilized for the TCM and TTCM scheme. Different modulation schemes are employed, namely, binary phase shift keying (BPSK), quadrature phase shift keying (QPSK), 8-level phase shift keying (8PSK), 16-level quadrature amplitude modulation (16QAM), and 64-level quadrature amplitude modulation (64QAM) [101].

Following the mapper, the channel-coded symbols are passed to the STB encoder, as shown in Fig. 7. Below, we will investigate the performance of all the previously

$$
\mathbf{H}_{4}=\left(\begin{array}{cccc}
x_{1} & x_{2} & \frac{x_{3}}{\sqrt{2}} & \frac{x_{3}}{\sqrt{2}} \\
-\bar{x}_{2} & \bar{x}_{1} & \frac{x_{3}}{\sqrt{2}} & -\frac{x_{3}}{\sqrt{2}} \\
\bar{x}_{3} & \frac{\bar{x}_{3}}{\sqrt{2}} & \frac{\left(-x_{1}-\bar{x}_{1}+x_{2}-\bar{x}_{2}\right)}{2} & \frac{\left(-x_{2}-\bar{x}_{2}+x_{1}-\bar{x}_{1}\right)}{2} \\
\frac{\sqrt{2}}{\bar{x}_{3}} & -\frac{\bar{x}_{3}}{\sqrt{2}} & \frac{\left(x_{2}+\bar{x}_{2}+x_{1}-\bar{x}_{1}\right)}{2} & \frac{\left(-x_{1}-\bar{x}_{1}-x_{2}+\bar{x}_{2}\right)}{2}
\end{array}\right)
$$


mentioned STB codes, namely, that of the $\mathbf{G}_{2}, \mathbf{G}_{3}, \mathbf{G}_{4}, \mathbf{H}_{3}$, and $\mathbf{H}_{4}$ codes proposed in [23]-[25]. The corresponding transmission matrices are given in (10) and (31)-(34), respectively. The coding rate and number of transmitters of the associated STB codes is shown in Table 2. The channels are uncorrelated (or synonymously perfectly interleaved) narrow-band or nondispersive Rayleigh fading channels. This assumption does not contradict to requiring a constant channel magnitude and phase over $p$ (number of rows in the transmission matrix) consecutive symbols, since upon applying a sufficiently long channel interleaving depth, the channels' fading envelope can indeed become near uncorrelated. We assumed that the narrow-band fading amplitudes received from each transmitter antenna are mutually uncorrelated Rayleigh distributed processes. The average signal power received from each transmitter antenna is the same. Furthermore, we assume that the receiver has a perfect estimate of the channels' fading amplitudes. In practice, the channels' fading amplitude can be estimated, e.g., with the aid of pilot symbols [101].

At the receiver, the number of receiver antennas constitutes a design parameter, which is fixed to one unless specified otherwise. The STB decoders apply the MAP or Log-MAP decoding algorithm of [102] for the decoding of the signals received from the different antennas. Due to its implementational simplicity, the Log-MAP decoding algorithm is preferred in the proposed system. The soft outputs associated with the received bits or symbols are passed through the channel deinterleaver or directly to the TCM/TTCM decoder, respectively, as seen in Fig. 7. The channel-deinterleaved soft outputs of the received bits are then passed to the CC, TC, or TBCH decoders. The VA [34], [35] is applied in the CC and TCM decoder. By contrast, all turbo decoder schemes apply the Log-MAP [39], [80], [84] decoding algorithm. The decoded bits are finally passed to the information sink for calculation of the BER, as shown in Fig. 7.

\section{B. Channel-Codec Parameters}

In Fig. 7, we have given an overview of the proposed system. As we can see in Fig. 7, there are different channel encoders to be considered, namely, the $\mathrm{CC}, \mathrm{TC}, \mathrm{TBCH}$, TCM, and TTCM schemes. In this section, we present the parameters of all the channel codecs to be used in our investigations.

Table 3 shows the parameters of each channel encoder proposed in the system. We commence with the most well-known channel code, namely, the CC. A CC is described by three parameters $n, k$, and $K$ and it is denoted as $\mathrm{CC}(n, k, K)$. At each instant, a $\mathrm{CC}(n, k, K)$ encoder accepts $k$ input bits and outputs $n$ coded bits. The constraint length of the code is $K$ and the number of encoder states is equal to $2^{K-1}$. The channel-coded rate is given by

$$
R=\frac{k}{n}
$$

Different code rates can be obtained by suitable puncturing [106] and we will elaborate on this issue later in the section.
Table 3

Parameters of the Different Channel Encoders Used in Fig. 7

\begin{tabular}{l|c|c|c|c}
\hline Code & $\begin{array}{c}\text { Octal } \\
\text { generator } \\
\text { polynomial }\end{array}$ & $\begin{array}{c}\text { No. } \\
\text { of } \\
\text { states }\end{array}$ & $\begin{array}{c}\text { Decoding } \\
\text { algorithm }\end{array}$ & $\begin{array}{c}\text { No. } \\
\text { of } \\
\text { iterations }\end{array}$ \\
\hline Convolutional Code (CC) \\
\hline CC(2,1,5) & 23,33 & 16 & VA & - \\
\hline CC $(2,1,7)$ & 171,133 & 64 & VA & - \\
\hline CC(2,1,9) & 561,753 & 256 & VA & - \\
\hline \multicolumn{5}{|c}{ Turbo Convolutional Code (TC) } \\
\hline TC(2,1,3) & 7,5 & 4 & Log-Map & 8 \\
\hline TC(2,1,4) & 13,15 & 8 & Log-Map & 8 \\
\hline TC(2,1,5) & 23,35 & 16 & Log-Map & 8 \\
\hline \multicolumn{5}{|c}{ Turbo BCH Code (TBCH) } \\
\hline TBCH(31,26) & 45 & 32 & Log-Map & 8 \\
\hline TBCH(32,26) & 45 & 64 & Log-Map & 8 \\
\hline TBCH(31,21) & 3551 & 1024 & Log-Map & 8 \\
\hline TBCH(63,57) & 103 & 64 & Log-Map & 8 \\
\hline TBCH(127,120) & 211 & 128 & Log-Map & 8 \\
\hline \multicolumn{6}{|c|}{ Trellis Coded Modulation (TCM) } \\
\hline 8PSK-TCM & $103,30,66$ & 64 & VA & - \\
\hline 16QAM-TCM & $101,16,64$ & 64 & VA & - \\
\hline Turbo Trellis Coded Modulation (TTCM) \\
\hline 8PSK-TTCM & $11,2,4$ & 8 & Log-Map & 8 \\
\hline 16QAM-TTCM & $23,2,4,10$ & 16 & Log-Map & 8 \\
\hline
\end{tabular}

The first entry of Table 3 is the $\operatorname{CC} C C(2,1,5)$, which was adopted by the GSM standardization committee in 1982 [72], [107]. Then, in 1996, a more powerful CC, the $\mathrm{CC}(2,1,7)$ arrangement, was employed by the DVB [65] standard for television, sound, and data services. Recently, the Universal Mobile Telecommunication System (UMTS) proposed the use of the $\mathrm{CC}(2,1,9)$ scheme, which is also shown in Table 3. The implementation of this scheme is about 16 times more complex than that of the $\mathrm{CC}(2,1,5)$ scheme adopted by GSM some 15 years ago. This clearly shows that the advances of integrated circuit technology have substantially contributed to the performance improvement of mobile communication systems.

As mentioned earlier, a turbo encoder consists of two component encoders. Generally, two identical RSC codes are used. Berrou et al. [38], [39] used two constraint length $K=3$, RSC codes, each having four trellis states. We denote a TC code as $\mathrm{TC}(n, k, K)$, where $n, k$, and $K$ have their usual interpretations, as in CC. In [38] and [39], the MAP algorithm [37] was employed for iterative decoding. However, in our systems, the Log-MAP decoding algorithm [75] is utilized. The Log-MAP algorithm is a more attractive version of the MAP algorithm, since it operates in the logarithmic domain in order to reduce the computational complexity and to mitigate the numerical problems associated with the MAP algorithm [75]. The number of turbo iterations was set to eight, since this yielded a performance close to the optimum performance associated 
Table 4

Simulation Parameters Associated With the CC and TCM Channel Encoders in Fig. 7

\begin{tabular}{l|c|c|c|c|c}
\hline \multirow{2}{*}{ Code } & $\begin{array}{c}\text { Code } \\
\text { Rate }\end{array}$ & $\begin{array}{c}\text { Puncturing } \\
\text { Pattern }\end{array}$ & $\begin{array}{c}\text { Modula- } \\
\text { tion } \\
\text { Mode }\end{array}$ & BPS & $\begin{array}{c}\text { Random } \\
\text { interleaver } \\
\text { depth }\end{array}$ \\
\hline \multirow{6}{*}{ CC $(2,1,5)$} & 0.50 & 1,1 & QPSK & 1.00 & 20,000 \\
\hline CC(2,1,7) & 0.50 & 1,1 & QPSK & 1.00 & 20,000 \\
\cline { 2 - 6 } & 0.75 & 101,110 & 64QAM & 4.50 & 13,320 \\
\cline { 2 - 6 } & 0.83 & 10101,11010 & 64QAM & 5.00 & 12,000 \\
\hline CC(2,1,9) & 0.50 & 1,1 & QPSK & 1.00 & 20,000 \\
\cline { 2 - 6 } & & & 16QAM & 2.00 & 20,000 \\
\cline { 2 - 6 } & & & 64QAM & 3.00 & 20,004 \\
\hline Trellis Coded Modulation (TCM) & 8PSK & 2.00 & - \\
\hline 16QAM-TCM & 0.75 & 1,1 & 16QAM & 3.00 & - \\
\hline
\end{tabular}

with an infinite number of iterations. In our investigations, we will consider the TC code TC $(2,1,3)$, proposed in [38], [39]. However, the more complex TC $(2,1,4)$ code [108] was proposed by UMTS to be employed in the $3 \mathrm{G}$ mobile communication systems [1], [72], [109]. The TC(2,1,5) code is also interesting, since it is expected to provide further significant coding gains over that of the $\mathrm{TC}(2,1,3)$ and $\mathrm{TC}(2,1,4)$ code.

$\mathrm{BCH}$ codes [72] are used as the component codes in the TBCH codes of Table 3. Again, TBCH codes have been shown, e.g., by Hagenauer et al. [84] and Nikl et al. [86] to perform impressively at near-unity coding rates, although at high complexity. Hence, in our study, the $\mathrm{BCH}$ component codes $\mathrm{BCH}(31,26), \mathrm{BCH}(31,21), \mathrm{BCH}(63,57)$, and $\mathrm{BCH}(127,120)$ are employed, as shown in Table 3. Finally, we also investigate TCM and TTCM. Both of them are employed in 8PSK and 16QAM modulation modes. This results in 8PSK-TCM, 16QAM-TCM, 8PSK-TTCM, and 16QAM-TTCM, respectively.

In Table 3, we give the encoding and decoding parameters of the different channel encoders employed. However, as mentioned earlier, we can design codes of variable code rates $R$ by employing suitable puncturing patterns. By combining puncturing with different modulation modes, we could design a system having a range of various throughputs, expressed in terms of the number of bits per symbol (BPS), as shown in Tables 4 and 5. Some of the parameters in Tables 4 and 5 are discussed in depth during our further discourse, but significantly more information can be gleaned concerning these systems by carefully studying both tables.

In Table 4, we summarize the simulation parameters of the CC and TCM schemes employed. Since there are two coded bits $(n=2)$ for each data bit $(k=1)$, we have two possible puncturing patterns, as shown in Table 4 . A binary 1 means that the coded bit is transmitted, whereas a binary 0 implies that the coded bit is punctured. Accordingly, the puncturing pattern $(1,1)$ simply implies that no puncturing is applied and, hence, results in a half-rate CC. However, in the DVB standard [65], different puncturing patterns were proposed for the $\mathrm{CC}(2,1,7)$ code, which result in different coding rates. These are also shown in Table 4.

In Table 5, the simulation parameters of three different turbo schemes, namely, those of the TC, TBCH, and TTCM arrangements are given. Again, different code rates can be designed using suitable puncturing patterns, where the puncturing patterns seen in Table 5 consist of two parts. Specifically, the associated different puncturing patterns represent the puncturing patterns of the parity bits emanating from the first and the second encoder, respectively. These patterns are different from the puncturing patterns seen in Table 4. For the $\mathrm{TC}(2,1,3)$ scheme, different puncturing patterns are employed for the various code rates $R$. The puncturing patterns were optimized experimentally by simulation in order to attain the best possible BER performance. The design procedure for punctured turbo codes was proposed by Acikel et al. [87] in the context of BPSK and QPSK.

\section{Complexity Issues and Memory Requirements}

In this section, the complexity issues and memory requirements of the proposed system are addressed. We will mainly focus on the relative estimated complexity and memory requirements of the proposed channel decoders rather than attempting to determine their exact complexity. Therefore, several assumptions are made in order to simplify our comparative study. In our simplified approach, the estimated complexity of the whole system is deemed to depend only on that of the channel decoders. In other words, the complexity associated with the modulator, demodulator, space-time encoder, and decoder as well as channel encoders are assumed to be insignificant compared to the complexity of channel decoders.

Since the estimated complexity of the channel decoders depends directly on the number of trellis transitions, the number of trellis transitions per information data bit will be used as the basis of our comparison. Several channel encoder schemes in Table 3 are composed of CCs. For the binary $\mathrm{CC} \mathrm{CC}(2,1, K)$, two trellis transitions diverge from each of the $2^{K-1}$ states. Hence, we can approximate the complexity of a $\mathrm{CC}(2,1, K)$ code as

$$
\begin{aligned}
\operatorname{comp}\{\mathrm{CC}(2,1, K)\} & =2 \times 2^{K-1} \\
& =2^{K} .
\end{aligned}
$$

The number of trellis transitions in the Log-MAP decoding algorithm is assumed to be three times greater, than that of the conventional VA, since the Log-MAP algorithm has to perform forward as well as backward recursion and soft output calculations, which results in traversing through the trellis three times. The reader is referred to [37] for further details of the algorithm. For TC codes, we apply the Log-MAP decoding algorithm for iterative decoding, assisted by the two component decoders. Upon taking into account the number of turbo decoding iterations as well, the complexity of TC decoding is approximated by

$$
\begin{aligned}
\operatorname{comp}\{\mathrm{TC}(2,1, K)\} & =3 \times 2 \times 2^{K-1} \times 2 \times \text { No. of Iterations } \\
& =3 \times 2^{K+1} \times \text { No. of Iterations. }
\end{aligned}
$$


Table 5

Simulation Parameters Associated With the TC, TBCH, and TTCM Channel Encoders in Fig. 7

\begin{tabular}{|c|c|c|c|c|c|c|}
\hline Code & $\begin{array}{c}\text { Code } \\
\text { Rate } \\
R\end{array}$ & $\begin{array}{l}\text { Puncturing } \\
\text { Pattern }\end{array}$ & $\begin{array}{l}\text { Modula- } \\
\text { tion } \\
\text { Format }\end{array}$ & BPS & $\begin{array}{c}\text { Random } \\
\text { turbo } \\
\text { interleaver } \\
\text { depth }\end{array}$ & $\begin{array}{c}\text { Random } \\
\text { (separation) } \\
\text { interleaver } \\
\text { depth }\end{array}$ \\
\hline \multicolumn{7}{|c|}{ Turbo Convolutional Code (TC) } \\
\hline $\mathrm{TC}(2,1,3)$ & 0.50 & 10,01 & 16QAM & 2.00 & 10,000 & 20,000 \\
\hline \multirow[t]{8}{*}{$\mathrm{TC}(2,1,4)$} & 0.33 & 1,1 & 64QAM & 2.00 & 10,000 & 30,000 \\
\hline & \multirow[t]{2}{*}{0.50} & \multirow[t]{2}{*}{10,01} & 16QAM & 2.00 & 10,000 & 20,000 \\
\hline & & & 64QAM & 3.00 & 10,002 & 20,004 \\
\hline & 0.67 & 1000,0001 & 64QAM & 4.00 & 10,000 & 15,000 \\
\hline & \multirow[t]{2}{*}{0.75} & \multirow{2}{*}{$\begin{array}{l}100000 \\
000001\end{array}$} & 16QAM & 3.00 & 9,990 & 13,320 \\
\hline & & & 64QAM & 4.50 & 9,990 & 13,320 \\
\hline & 0.83 & $\begin{array}{l}1000000000 \\
0000000001\end{array}$ & 64QAM & 5.00 & 10,000 & 12,000 \\
\hline & 0.90 & $\begin{array}{l}100000000000000000 \\
000000000000000001\end{array}$ & 64QAM & 5.40 & 10,044 & 11,160 \\
\hline $\mathrm{TC}(2,1,5)$ & 0.50 & 10,01 & 16QAM & 2.00 & 10,000 & 20,000 \\
\hline \multicolumn{7}{|c|}{ Turbo BCH Code (TBCH) } \\
\hline \multirow[t]{2}{*}{$\mathrm{TBCH}(31,26)$} & \multirow[t]{2}{*}{0.72} & \multirow[t]{2}{*}{1,1} & 16QAM & 2.89 & 9,984 & 13,824 \\
\hline & & & 64QAM & 4.33 & 9,984 & 13,824 \\
\hline $\mathrm{TBCH}(32,26)$ & 0.68 & 1,1 & $8 \mathrm{PSK}$ & 2.05 & 9,984 & 14,592 \\
\hline $\operatorname{TBCH}(31,21)$ & 0.51 & 1,1 & 16QAM & 2.04 & 9,996 & 19,516 \\
\hline $\mathrm{TBCH}(63,57)$ & 0.83 & 1,1 & 64QAM & 4.96 & 10,032 & 12,144 \\
\hline $\mathrm{TBCH}(127,120)$ & 0.90 & 1,1 & 64QAM & 5.37 & 10,080 & 11,256 \\
\hline \multicolumn{7}{|c|}{ Turbo Trellis Coded Modulation (TTCM) } \\
\hline 8PSK-TTCM & $2 / 3$ & 10,01 & 8PSK & 2.00 & 10,000 & - \\
\hline 16QAM-TTCM & $3 / 4$ & 10,01 & 16QAM & 3.00 & 13,332 & - \\
\hline
\end{tabular}

In TCM, we construct a nonbinary decoding trellis [22]. The TCM schemes of Table 3 have $2^{\mathrm{BPS}-1}$ trellis branches diverging from each trellis state, where BPS is the number of transmitted bits per modulation symbol. However, for each trellis transition, we have BPS -1 transmitted information data bits, since the TCM encoder typically adds one parity bit per nonbinary symbol. Therefore, we can estimate the complexity of the proposed TCM schemes as

$$
\operatorname{comp}\{\mathrm{TCM}\}=2^{\mathrm{BPS}-1} \times \frac{\text { No. of States }}{\text { BPS }-1} .
$$

Similarly to TC, TTCM consists of two TCM codes and the Log-MAP decoding algorithm [80] is employed for iterative decoding. The associated TTCM complexity is then estimated as

$$
\begin{aligned}
& \operatorname{comp}\{\mathrm{TTCM}\} \\
& =3 \times 2^{\mathrm{BPS}-1} \times \frac{\text { No. of States }}{\text { BPS }-1} \times 2 \times \text { No. of Iterations } \\
& =\frac{3 \times 2^{\mathrm{BPS}} \times \text { No. of States } \times \text { No. of Iterations }}{\text { BPS }-1}
\end{aligned}
$$

For $\operatorname{TBCH}(n, k)$ codes, the complexity estimation is not as straightforward as in the previous cases. Its component codes are $\mathrm{BCH}(n, k)$ codes and the decoding trellis can be divided into three sections [44]. Assuming that $k>n-k$, for every decoding instant $j$, the number of trellis states is given [44]

No. of States $j= \begin{cases}2^{j}, & j=0,1, \ldots, n-k-1 \\ 2^{n-k}, & j=n-k, n-k+1, \ldots, k \\ 2^{n-j}, & j=k+1, k+2, \ldots, n .\end{cases}$

It can be readily shown that

$$
\begin{aligned}
2^{n-k}-1 & =\sum_{j=0}^{n-k-1} 2^{j} \\
& =\sum_{j=k+1}^{n} 2^{n-j} .
\end{aligned}
$$


Upon using the approximation $\sum_{j=0}^{n-k-1} 2^{j}=\sum_{j=k+1}^{n}$ $2^{n-j}=2^{n-k}-1 \approx 2^{n-k}$, we can write the number of decoding trellis states per information data bit as

$$
\begin{aligned}
\text { No. of States } & =\frac{2 \times 2^{n-k}+\{k-(n-k)\} \times 2^{n-k}}{k} \\
& =\frac{(2 k-n+2) \times 2^{n-k}}{k}
\end{aligned}
$$

Having derived the number of decoding trellis states per information data bit, we can approximate the complexity of TBCH codes as

$$
\begin{aligned}
& \operatorname{comp}\{\operatorname{TBCH}(n, k)\} \\
& =3 \times 2 \times \frac{(2 k-n+2) \times 2^{n-k}}{k} \times 2 \times \text { No. of Iterations } \\
& =\frac{3 \times(2 k-n+2) \times 2^{n-k+2} \times \text { No. of Iterations }}{k} .
\end{aligned}
$$

With the complexity of each channel decoder in hands, we will now derive their approximate memory requirements. Typically, the memory requirement of a channel decoder depends directly on the number of trellis states in the entire coded block. Therefore, in this section, the number of trellis states per coded block serves as the basis of a relative memory requirement comparison between the channel decoders studied. For a binary CC, observation of the VA has shown that typically all surviving paths of the current trellis state emerge from trellis states not "older" than approximately five times the constraint length $K$ [101]. Therefore, at any decoding instant, only a section of $5 \times K$ trellis transitions has to be stored. We can then approximate the associated memory requirement as

$$
\operatorname{mem}\{\mathrm{CC}(2,1, K)\}=2^{K-1} \times 5 \times K .
$$

Again, as highlighted in [37], the Log-MAP algorithm requires the storage of $\gamma, \alpha$, and $\beta$ values. Hence, for the same number of decoding trellis states, the Log-MAP algorithm requires about three times more memory than the classic VA. Consequently, we can estimate the memory requirement of the TC code as

$$
\operatorname{mem}\{\mathrm{TC}(2,1, K)\}=3 \times 2^{K-1} \times \text { Block Length. }
$$

Similarly to CCs, we can approximate the memory requirements of TCM as

$$
\operatorname{mem}\{\mathrm{TCM}\}=\text { No. of States } \times \text { Block Length. }
$$

Following similar arguments, the memory requirements of TTCM employing the Log-MAP algorithm can be approximated as

$$
\operatorname{mem}\{\text { TTCM }\}=3 \times \text { No. of States } \times \text { Block Length. }
$$

The estimation of the memory requirements of $\mathrm{TBCH}$ codes is again different from that of the other channel codes considered. Specifically, their memory requirement does not directly depend on the number of decoding trellis states in a coded TBCH block. Instead, it depends on the number of decoding trellis states in the constituent $\mathrm{BCH}$ codewords. From (43), we can estimate the associated memory requirements as

$$
\operatorname{mem}\{\operatorname{TBCH}(n, k)\}=3 \times(2 k-n+2) \times 2^{n-k} .
$$

Applying (36)-(49), we summarize the estimated complexity and memory requirements of the channel decoders characterized in Table 3. Explicitly, assuming that there are 10000 information data bits per coded block, the associated estimated complexity and memory requirements are then given in Table 6. Note that the block length of TCM and TTCM is expressed in terms of the number of symbols per coded block, since these schemes are symbol-oriented rather than bit-oriented.

\section{PERFORMANCE RESUlTS}

In this section, unless otherwise stated, all simulation results are obtained over uncorrelated (or perfectly interleaved) narrow-band or nondispersive Rayleigh fading channels. As stated before, this does not contradict requiring a constant channel magnitude and phase over $n$ consecutive time slots in (8), since upon applying a sufficiently high interleaving depth, the channel's fading envelope can be indeed uncorrelated. Our assumptions are that:

1) the fading amplitudes are constant across $n$ consecutive transmission slots of the STB codes' transmission matrix;

2) the average signal power received from each transmitter antenna is the same;

3) the receiver has perfect knowledge of the channels' fading amplitudes.

We note that the above assumptions are unrealistic, yielding the best-case performance, nonetheless facilitating the performance comparison of the various techniques under identical circumstances.

In the following sections, we compare the performance of various combinations of STB codes and channel codes. As mentioned earlier, various code rates can be used for both the STB codes and for the associated channel codes. The different modulation schemes employed result in various effective throughputs. Hence, for a fair comparison, all different systems are compared on the basis of the same effective BPS throughput given by

$$
\mathrm{BPS}=R_{\mathrm{st}} \times R_{\mathrm{cc}} \times \text { modulation throughput }
$$

where $R_{\mathrm{st}}$ and $R_{\mathrm{cc}}$ are the code rates of the STB code and the channel code, respectively.

\section{A. Performance Comparison of Various STB Codes Without Channel Codecs}

In this section, the performances of various STB codes without channel codes are investigated and compared. All the investigated STB codes, namely, the $\mathbf{G}_{2}, \mathbf{G}_{3}, \mathbf{G}_{4}, \mathbf{H}_{3}$, and $\mathbf{H}_{4}$ codes [23]-[25] have their corresponding transmission matrices given in (10) and (31)-(34), respectively. The encoding parameters are summarized in Table 2. 
Table 6

Complexity and Memory Requirements of the Different Channel Decoders Characterized in Table 3

\begin{tabular}{|c|c|c|c|c|c|c|}
\hline Code & $\begin{array}{c}\text { No. } \\
\text { of } \\
\text { states }\end{array}$ & $\begin{array}{c}\text { No. of } \\
\text { states per } \\
\text { data bit }\end{array}$ & $\begin{array}{c}\text { Iteration } \\
\text { No }\end{array}$ & $\begin{array}{l}\text { Block } \\
\text { length }\end{array}$ & Complexity & $\begin{array}{l}\text { Memory } \\
\text { requirement }\end{array}$ \\
\hline \multicolumn{7}{|c|}{ Convolutional Code (CC) } \\
\hline $\mathrm{CC}(2,1,5)$ & 16 & 16 & - & 10,000 & 32 & 400 \\
\hline $\mathrm{CC}(2,1,7)$ & 64 & 64 & - & 10,000 & 128 & 2,240 \\
\hline $\mathrm{CC}(2,1,9)$ & 256 & 256 & - & 10,000 & 512 & 11,520 \\
\hline \multicolumn{7}{|c|}{ Turbo Convolutional Code (TC) } \\
\hline $\mathrm{TC}(2,1,3)$ & 4 & 4 & 8 & 10,000 & 384 & 120,000 \\
\hline $\mathrm{TC}(2,1,4)$ & 8 & 8 & 8 & 10,000 & 768 & 240,000 \\
\hline $\mathrm{TC}(2,1,5)$ & 16 & 16 & 8 & 10,000 & 1,536 & 480,000 \\
\hline \multicolumn{7}{|c|}{ Turbo BCH Code (TBCH) } \\
\hline TBCH $(31,26)$ & 32 & 28 & 8 & 31 & 2,718 & 2,208 \\
\hline $\mathrm{TBCH}(32,26)$ & 64 & 54 & 8 & 32 & 5,199 & 4,224 \\
\hline TBCH $(31,21)$ & 1,024 & 634 & 8 & 31 & 60,855 & 39,936 \\
\hline $\operatorname{TBCH}(63,57)$ & 64 & 60 & 8 & 63 & 5,713 & 10,176 \\
\hline $\mathrm{TBCH}(127,120)$ & 128 & 123 & 8 & 127 & 11,776 & 44,160 \\
\hline \multicolumn{7}{|c|}{ Trellis Coded Modulation (TCM) } \\
\hline 8PSK-TCM & 64 & 32 & - & 5,000 & 128 & 320,000 \\
\hline 16QAM-TCM & 64 & 21 & - & 3,333 & 171 & 213,312 \\
\hline \multicolumn{7}{|c|}{ Turbo Trellis Coded Modulation (TTCM) } \\
\hline 8PSK-TTCM & 8 & 4 & 8 & 5,000 & 768 & 120,000 \\
\hline 16QAM-TTCM & 16 & 5 & 8 & 3,333 & 2,048 & 159,984 \\
\hline
\end{tabular}

1) Maximum Ratio Combining and the Space-Time Code $\mathbf{G}_{2}$ : Fig. 8 shows the performance of MRC and the space-time code $\mathbf{G}_{2}$ using BPSK over uncorrelated Rayleigh fading channels. It is assumed that the total power received from both transmit antennas in the space-time-coded system using $\mathbf{G}_{2}$ of (10) is the same as the transmit power of the single transmit antenna assisted MRC system. It can be seen in Fig. 8 that the performance of the space-time code $\mathbf{G}_{2}$ is about $3 \mathrm{~dB}$ worse than that of the MRC technique using two receivers, even though both systems have the same diversity order of two- the $\mathbf{G}_{2}$ code uses two transmitters, while the MRC scheme two receivers. The 3-dB penalty is incurred because the transmit power of each antenna in the $\mathbf{G}_{2}$ space-time-coded arrangement is only half of the transmit power in the MRC assisted system. It is shown in Fig. 8, however, that at a BER of $10^{-5}$, a diversity gain of $20 \mathrm{~dB}$ is achieved by the space-time code $\mathbf{G}_{2}$. If we increase the diversity order to four by using two receivers, the space-time code $\mathbf{G}_{2}$ achieves a diversity gain of 32 $\mathrm{dB}$. However, there is still a $3-\mathrm{dB}$ performance penalty as compared to the conventional MRC technique using four receivers. The advantage of the space-time-coded scheme is nonetheless that the increased complexity of the space-time-coded transmitter is more affordable at the BS than at the MS, where the MRC receiver would have to be located.

\section{BER against $\mathrm{E}_{\mathrm{b}} / \mathrm{N}_{0}$}

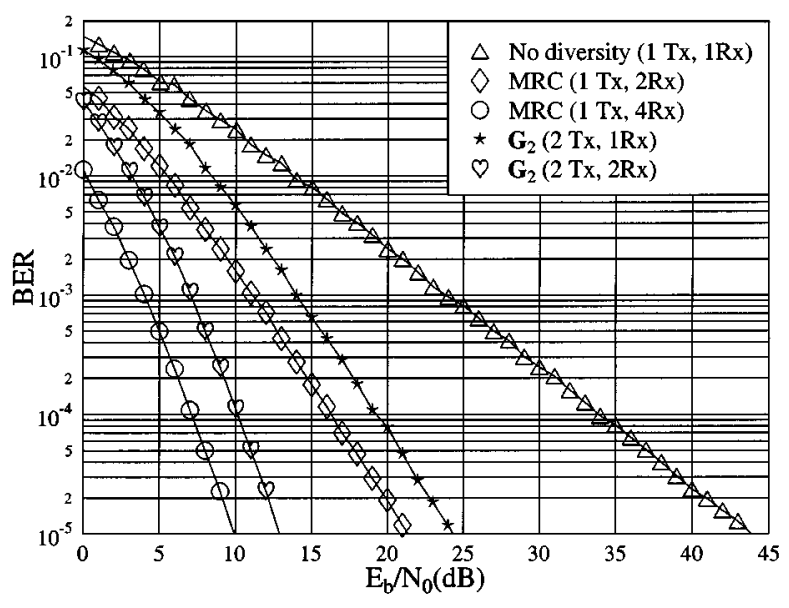

Fig. 8. Performance comparison of the MRC technique and space-time code $\mathbf{G}_{2}$ using BPSK over uncorrelated Rayleigh fading channels.

2) Performance of 1-BPS Schemes: Figs. 9 and 10 compare the performances of the space-time codes $\mathbf{G}_{2}, \mathbf{G}_{3}$, and $\mathbf{G}_{4}$ having an effective throughput of 1 BPS over uncorrelated Rayleigh fading channels using one and two receivers, respectively. BPSK modulation was employed in conjunction with the space-time code $\mathbf{G}_{2}$. As shown in Table 2, the 


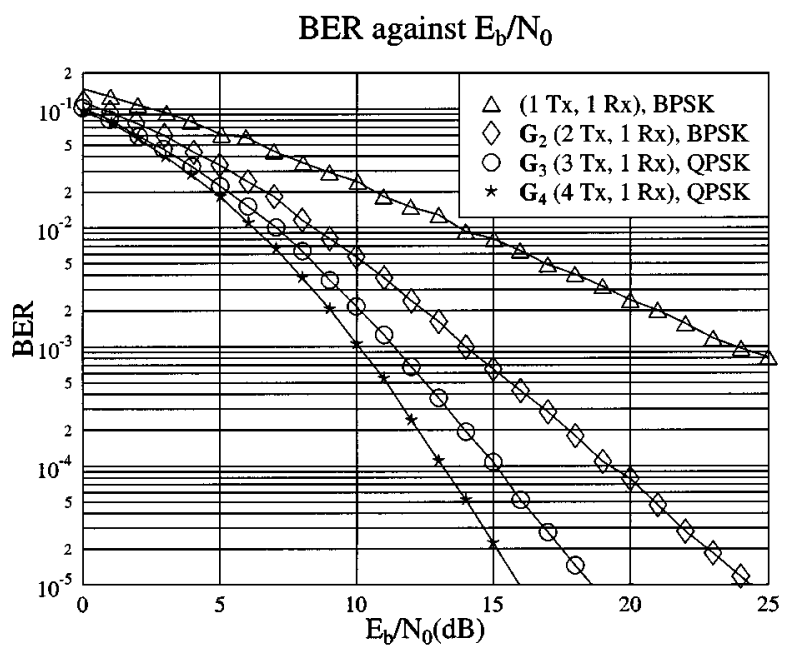

Fig. 9. Performance comparison of the space-time codes $\mathbf{G}_{2}, \mathbf{G}_{3}$ and $\mathbf{G}_{4}$ of Table 2 at an effective throughput of 1 BPS using one receiver over uncorrelated Rayleigh fading channels.

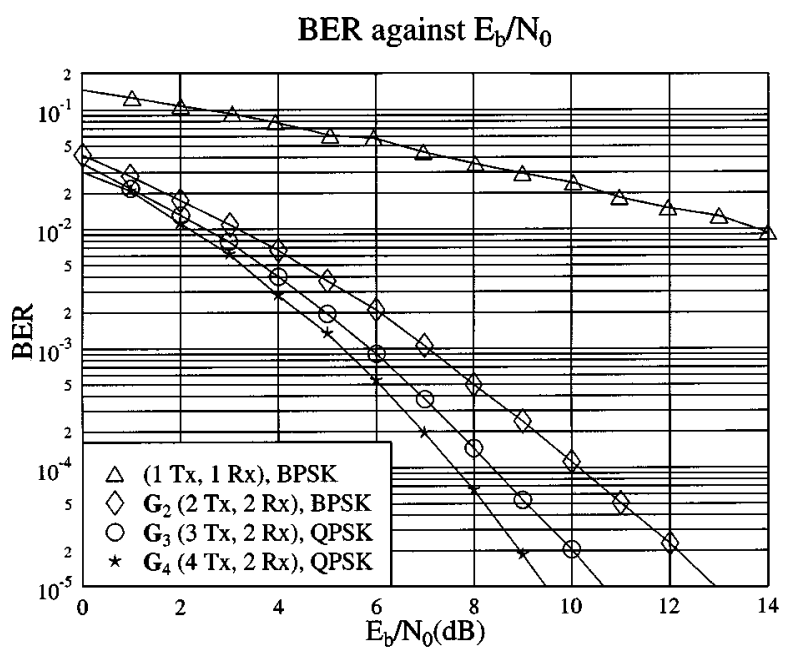

Fig. 10. Performance comparison of the space-time codes $\mathbf{G}_{2}$, $\mathbf{G}_{3}$, and $\mathbf{G}_{4}$ of Table 2 at an effective throughput of 1 BPS using two receivers over uncorrelated Rayleigh fading channels.

space-time codes $\mathbf{G}_{3}$ and $\mathbf{G}_{4}$ are half-rate codes. Therefore, QPSK modulation was used in the context of $\mathbf{G}_{3}$ and $\mathbf{G}_{4}$ in order to retain a throughput of 1 BPS. It can be seen in Fig. 9 that at a BER of $10^{-5}$, the space-time codes $\mathbf{G}_{3}$ and $\mathbf{G}_{4}$ give about 5- and 7.5-dB gain over the $\mathbf{G}_{2}$ code, respectively. If the number of receivers is increased to two, as shown in Fig. 10, the associated $E_{b} / N_{0}$ gain reduces to about 1- and $3.5-\mathrm{dB}$, respectively. The reason is that over the perfectly interleaved flat-fading channel considered much of the attainable diversity gain is already achieved using the $\mathbf{G}_{2}$ code and two receivers. The associated gains of the various schemes at a BER of $10^{-5}$ are summarized in Table 7.

3) Performance of 2-BPS Schemes: In Fig. 11, we compare the performances of the space-time codes $\mathbf{G}_{2}, \mathbf{G}_{3}, \mathbf{G}_{4}, \mathbf{H}_{3}$ and $\mathbf{H}_{4}$ proposed in [23]-[25] using the encoding parameters summarized in Table 2. The performance results were obtained over uncorrelated Rayleigh fading channels using one receiver and the effective throughput of the system is about 2 BPS. For the $\mathbf{G}_{2}$ code,
Table 7

Coding Gain of the STB Codes of Table 2 Over Uncorrelated Rayleigh Fading Channels at BER $=10^{-5}$

\begin{tabular}{l|c|c|c|c|c|c|c}
\hline \multicolumn{2}{c|}{} & \multicolumn{4}{c|}{ One receiver } & \multicolumn{3}{c}{ Two receivers } \\
\hline Code & Rate & 1 BPS & 2 BPS & 3 BPS & 1 BPS & 2 BPS & 3 BPS \\
\hline $\mathbf{G}_{2}$ & 1 & 19.5 & 19.6 & 19.1 & 30.9 & 30.9 & 30.1 \\
\hline $\mathbf{G}_{3}$ & $1 / 2$ & 25.2 & 21.8 & 20.0 & 33.2 & 29.6 & 27.6 \\
\hline $\mathbf{G}_{4}$ & $1 / 2$ & 27.9 & 24.3 & 22.4 & 34.3 & 30.7 & 28.8 \\
\hline $\mathbf{H}_{3}$ & $3 / 4$ & - & 22.4 & 24.0 & - & 30.1 & 31.9 \\
\hline $\mathbf{H}_{4}$ & $3 / 4$ & - & 24.8 & 22.6 & - & 31.2 & 33.0 \\
\hline
\end{tabular}

\section{BER against $\mathrm{E}_{\mathrm{b}} / \mathrm{N}_{0}$}

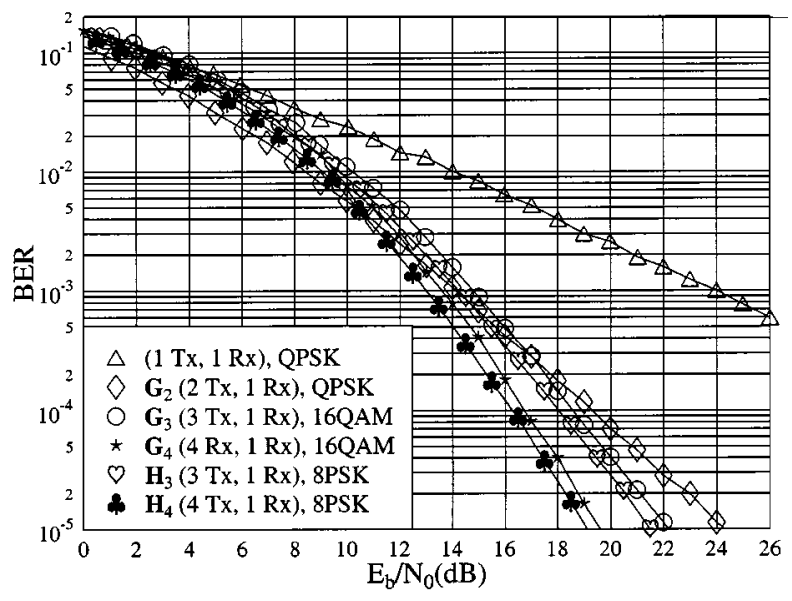

Fig. 11. Performance comparison of the space-time codes $\mathbf{G}_{2}$, $\mathbf{G}_{3}, \mathbf{G}_{4}, \mathbf{H}_{3}$, and $\mathbf{H}_{4}$ at an effective throughput of approximately 2 BPS using one receiver over uncorrelated Rayleigh fading channels. Associated parameters of the space-time codes are summarized in Table 2.

QPSK modulation is used, while the $\mathbf{G}_{3}$ and $\mathbf{G}_{4}$ codes employ 16QAM conveying 4 BPS. Hence, the effective throughput is 2 BPS, since $\mathbf{G}_{3}$ and $\mathbf{G}_{4}$ are half-rate codes. Since the code rate of the $\mathbf{H}_{3}$ and $\mathbf{H}_{4}$ codes is $3 / 4$, 8PSK modulation was employed in this context, resulting in a throughput of $3 \times 3 / 4=2.25 \mathrm{BPS}$, which is approximately 2 BPS. We can see in Fig. 11 that at high BERs or low $E_{b} / N_{0}$ values, the $\mathbf{G}_{2}$ code slightly outperforms the others. However, the situation is reversed when the system is operated at a low BER or high $E_{b} / N_{0}$ values. At a BER of $10^{-5}$, the code $\mathbf{G}_{4}$ only gives a diversity gain of $5 \mathrm{~dB}$ over the $\mathbf{G}_{2}$ code. This is a $2.5-\mathrm{dB}$ loss compared to the $7.5-\mathrm{dB}$ gain achieved by the system transmitting at an effective throughput of 1 BPS in the previous section. This is because the more vulnerable 16QAM scheme was used for the space-time code $\mathbf{G}_{4}$. Since the 16QAM signal constellation is more densely packed compared to QPSK, it is more prone to errors. Moreover, the space-time code $\mathbf{G}_{4}$ has no error correction capability to correct the extra errors induced by employing a more vulnerable higher order modulation scheme. Hence, this results in a poorer performance. If the throughput of the system is increased by employing a higher order modulation scheme, the space-time code $\mathbf{G}_{4}$ will suffer even higher performance degradations, as shown 
BER against $\mathrm{E}_{\mathrm{b}} / \mathrm{N}_{0}$

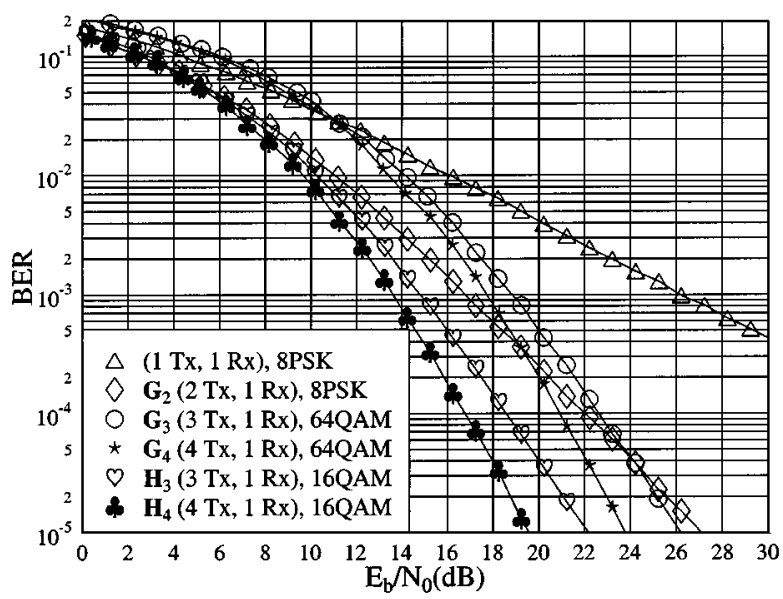

Fig. 12. Performance comparison of the space-time codes $\mathbf{G}_{2}$ $\mathbf{G}_{3}, \mathbf{G}_{4}, \mathbf{H}_{3}$, and $\mathbf{H}_{4}$ of Table 2 at an effective throughput of 3 BPS using one receiver over uncorrelated Rayleigh fading channels.

Section VI-A4. Since the space-time code $\mathbf{G}_{3}$ of Table 2 is also a half-rate code, similarly to the $\mathbf{G}_{4}$ code, it suffers from the same drawbacks.

In Fig. 11, we also show the performance of the threequarter rate space-time codes $\mathbf{H}_{3}$ and $\mathbf{H}_{4}$ of Table 2. Both the $\mathbf{H}_{4}$ and $\mathbf{G}_{4}$ codes have the same diversity order of four in conjunction with one receiver. However, at a BER of $10^{-5}$, the performance of the $\mathbf{H}_{4}$ code is about $0.5 \mathrm{~dB}$ better than that of the $\mathbf{G}_{4}$ code. This is again due to the higher order modulation employed in conjunction with the half-rate code $\mathbf{G}_{4}$ in order to maintain the same throughput. As noted earlier, the higher order modulation schemes are more susceptible to errors and hence the performance of the system in conjunction with the $\mathbf{G}_{3}$ or $\mathbf{G}_{4}$ code of Table 2 is worse than that of the $\mathbf{H}_{3}$ or $\mathbf{H}_{4}$ code having the same diversity order, respectively. The associated gains of the various schemes at a BER of $10^{-5}$ are summarized in Table 7 .

4) Performance of 3-BPS Schemes: Figs. 12 and 13 show performance comparisons for the space-time codes $\mathbf{G}_{2}, \mathbf{G}_{3}$, $\mathbf{G}_{4}, \mathbf{H}_{3}$, and $\mathbf{H}_{4}$ of Table 2 at an effective throughput of 3 BPS over uncorrelated Rayleigh fading channels using one and two receivers, respectively. When using the $\mathbf{G}_{2}$ code, we employ 8PSK modulation. Since $\mathbf{G}_{3}$ and $\mathbf{G}_{4}$ are half-rate codes, 64QAM is employed in order to obtain an effective throughput of 3 BPS. By contrast, for the $\mathbf{H}_{3}$ and $\mathbf{H}_{4}$ codes, which have a code rate of 3/4, 16QAM was used in order to ensure the same throughput of $4 \times 3 / 4=3$ BPS.

In Fig. 12 we can see that at a BER of $10^{-5}$, the diversity gain of the $\mathbf{G}_{4}$ code over the $\mathbf{G}_{2}$ code is further reduced to about $3 \mathrm{~dB}$. There is only a marginal diversity gain for the $\mathbf{G}_{3}$ code over the $\mathbf{G}_{2}$ code. As alluded to in the previous section, 64QAM in conjunction with the space-time code $\mathbf{G}_{3}$ or $\mathbf{G}_{4}$ has a densely packed signal constellation and, hence, this scheme is prone to errors. At the higher BER of $10^{-2}$, the $\mathbf{G}_{2}$ code outperforms the $\mathbf{G}_{3}$ and $\mathbf{G}_{4}$ codes by approximately 3 and $4 \mathrm{~dB}$, respectively.

Due to the associated higher order modulation scheme employed, we can see in Fig. 12 that at a BER of $10^{-5}$, the $\mathbf{H}_{3}$

\section{BER against $\mathrm{E}_{\mathrm{b}} / \mathrm{N}_{0}$}

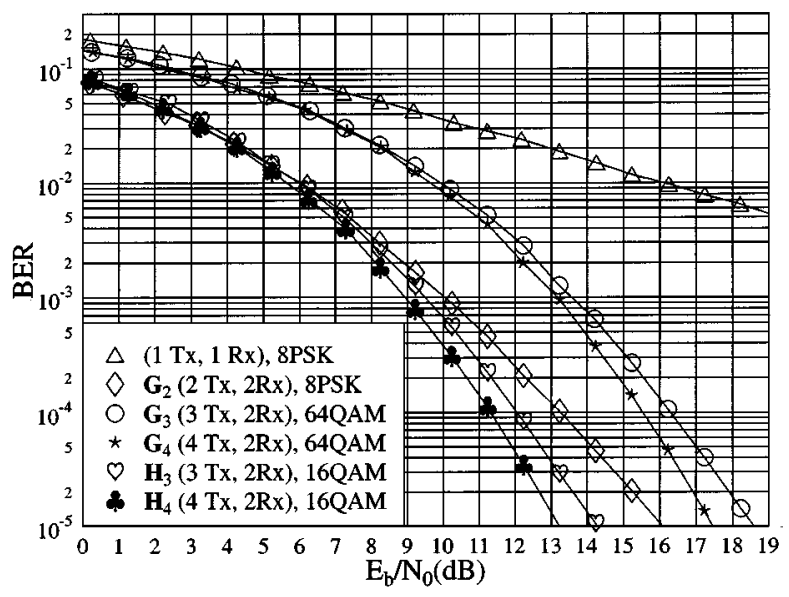

Fig. 13. Performance comparison of the space-time codes $\mathbf{G}_{2}$, $\mathbf{G}_{3}, \mathbf{G}_{4}, \mathbf{H}_{3}$, and $\mathbf{H}_{4}$ of Table 2 at an effective throughput of 3 BPS using two receivers over uncorrelated Rayleigh fading channels.

and $\mathbf{H}_{4}$ codes of Table 2 outperform both the $\mathbf{G}_{3}$ and the $\mathbf{G}_{4}$ codes. Specifically, we can see that the $\mathbf{H}_{3}$ code attains about 2-dB gain over the $\mathbf{G}_{4}$ code, even though it has a lower diversity order.

If we increase the number of receivers to two, a scenario characterized in Fig. 13, the performance degradation of the space-time codes $\mathbf{G}_{3}$ and $\mathbf{G}_{4}$ is even more pronounced. At a BER of $10^{-5}$, the performance gain of the $\mathbf{H}_{4}$ code over the $\mathbf{G}_{4}$ code is approximately $4 \mathrm{~dB}$ compared to the $0.5-\mathrm{dB}$ gain, when the system's effective throughput is only 2 BPS, as shown in Fig. 11.

Studying Figs. 9-13, we may conclude two important points. First, the space-time codes $\mathbf{G}_{3}$ and $\mathbf{G}_{4}$ of Table 2 suffer from having a code rate of half, since this significantly reduces the effective throughput of the system. In order to maintain the same throughput as the unity rate $\mathbf{G}_{2}$ code, higher order modulation schemes, such as 64QAM, have to be employed. This results in more channel errors, since the constellation points of the higher order modulation schemes are more densely packed. Due to their lack of error correcting capability, the $\mathbf{G}_{3}$ and $\mathbf{G}_{4}$ codes suffer performance losses compared to the $\mathbf{G}_{2}$ code. Second, if the number of receivers is increased to two, the performance gain of the $\mathbf{G}_{3}, \mathbf{G}_{4}, \mathbf{H}_{3}$, or $\mathbf{H}_{4}$ codes over the $\mathbf{G}_{2}$ code becomes smaller. The reason behind this phenomenon is that much of the attainable diversity gain was already achieved using the $\mathbf{G}_{2}$ code and two receivers. The associated gains of the various schemes at a BER of $10^{-5}$ are summarized in Table 7.

5) Channel-Coded STB Codes: In the previous sections, we have shown that without channel coding, the performance of the unity-rate space-time $\mathbf{G}_{2}$ code is inferior to the lower rate space-time codes, namely, to that of the $\mathbf{G}_{3}, \mathbf{G}_{4}, \mathbf{H}_{3}$, and $\mathbf{H}_{4}$ schemes. Since the space-time code $\mathbf{G}_{2}$ has a unity code rate, half-rate turbo codes can be employed for improving the performance of the system. In Fig. 14, we compare the performance of the half-rate $\mathrm{TC}(2,1,4)$ code concatenated with the space-time code $\mathbf{G}_{2}$ and with the STB 


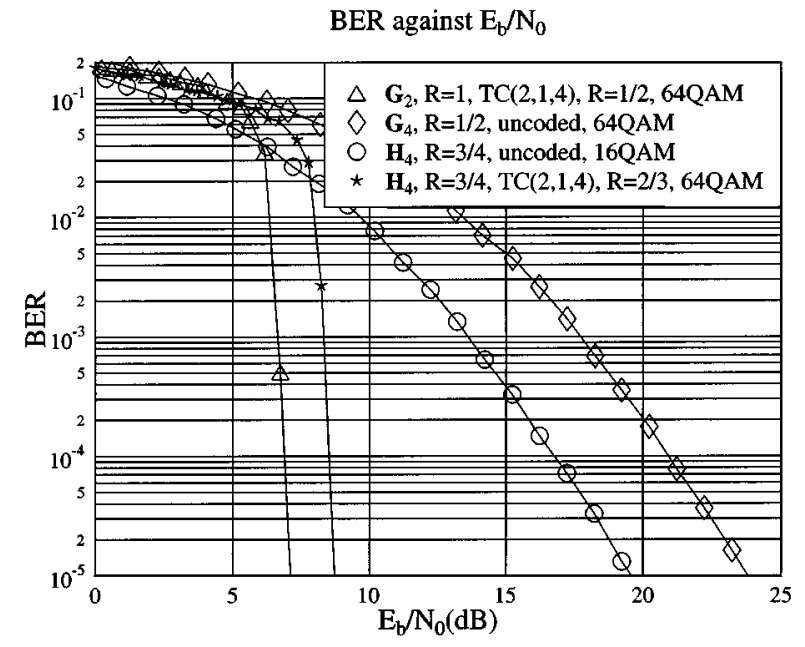

Fig. 14. Performance comparison of the half-rate $\mathrm{TC}(2,1,4)$ code concatenated with the space-time code $\mathbf{G}_{2}$ and the STB codes $\mathbf{G}_{4}$ and $\mathbf{H}_{4}$. Associated parameters are shown in Tables 2, 3, and 5. All simulation results were obtained at an effective throughput of 3 BPS over uncorrelated Rayleigh fading channels.

codes $\mathbf{G}_{4}$ and $\mathbf{H}_{4}$. Both the space-time codes $\mathbf{G}_{4}$ and $\mathbf{H}_{4}$ have a diversity gain of four and a code rate of $1 / 2$ and $3 / 4$, respectively. The associated parameters are shown in Tables 2,3 , and 5. Suitable modulation schemes were chosen so that all systems had the same throughput of 3 BPS. All simulation results were obtained over uncorrelated Rayleigh fading channels.

From Fig. 14, we can see that a huge performance improvement is achieved by concatenating the space-time code $\mathbf{G}_{2}$ with the half-rate code $\mathrm{TC}(2,1,4)$. At a BER of $10^{-5}$, this concatenated scheme attains a coding gain of 16 and $13 \mathrm{~dB}$ compared to the space-time codes $\mathbf{G}_{4}$ and $\mathbf{H}_{4}$, respectively. This clearly shows that it is better to invest the parity bits associated with the code-rate reduction in the concatenated turbo code, rather than in nonunity-rate STB codes. In Fig. 14, we also show the performance of the space-time code $\mathbf{H}_{4}$ concatenated with the punctured two-third-rate code $\mathrm{TC}(2,1,4)$. Fig. 14 shows that the $\mathrm{TC}(2,1,4)$ code improves the performance of the system tremendously, attaining a coding gain of $11 \mathrm{~dB}$ compared to the nonturbo-coded space-time code $\mathbf{H}_{4}$ at $\mathrm{BER}=10^{-\tilde{5}}$. However, its performance is still inferior to that of the half-rate $\mathrm{TC}(2,1,4)$-coded space-time code $\mathbf{G}_{2}$.

In conclusion, in Fig. 14, we have seen that the reduction in coding rate is best assigned to turbo channel codes, rather than space-time codes. Therefore, in all our forthcoming simulations, all channel codecs of Table 3 are concatenated with the unity-rate space-time code $\mathbf{G}_{2}$, instead of the nonunity-rate space-time codes $\mathbf{G}_{3}, \mathbf{G}_{4}, \mathbf{H}_{3}$, and $\mathbf{H}_{4}$ of Table 2.

\section{B. Mapping Binary Channel Codes to Multilevel Modulation}

As mentioned earlier in our investigations, different modulation schemes are employed in conjunction with the binary channel codecs $\mathrm{CC}$, TC, and TBCH. Specifically, the modulation schemes used are BPSK, QPSK, 8PSK, 16QAM, and 64QAM. Gray mapping [100], [101], [105] is employed to map the bits to the QPSK, 8PSK, 16QAM, and 64QAM symbols. In higher order modulation schemes, such as 8PSK, 16QAM, and 64QAM, there are several transmitted bits per constellation point. However, the different bit positions of the constellation points have different noise-protection distances [101]. More explicitly, the protection distance is the Euclidean distance from one constellation point to another, which results in the corruption of a particular bit. A larger noise-protection distance results in a higher integrity of the bit and vice versa. Therefore, for the different bit positions in the symbol, we have different protections for the transmitted bits within the phaser constellation of the nonbinary modulation schemes. It can be readily shown that in 8PSK and 16QAM, we have two protection classes, namely, class I and II [101], [105], where the class I transmitted bits are more protected. Similarly, in 64QAM, we have three protection classes, namely, I, II, and III [101], where the transmitted bits in class I are most protected, followed by class II and class III.

In our system, the parity bits are generated by binary channel encoders, such as the $\mathrm{CC}, \mathrm{TC}$, and $\mathrm{TBCH}$ schemes for protecting the binary data bits. However it is not intuitive whether the integrity of the data or parity bits is more important in yielding a better overall BER performance. For example, if the parity bits are more important, it is better to allocate the parity bits to the better protection classes in higher order modulation scheme and vice versa. Therefore, in this section, we will investigate the performance of different channel codes along with different bit mapping schemes. The effect of the bit interleaver seen in Fig. 7 is studied in conjunction with binary channel codes as well.

1) Turbo Convolutional Codes-Data and Parity Bit Mapping: We commence by studying half-rate TC codes, which are characterized in Table 3 . An equal number of parity and data bits are generated by the half-rate TC codes and they are mapped to the protection classes of the 16QAM scheme considered. Again, in the Gray-mapping-assisted 16QAM constellation, there are two protection classes [101], class I and II, depending on the bit position. Explicitly, there are 4 BPS in the 16QAM constellation and two of the bit positions are more protected than the remaining two bits.

In Fig. 15, we compare the performance of various parity and data bit mapping schemes for the: 1) $\mathrm{TC}(2,1,3) ; 2)$ $\mathrm{TC}(2,1,4)$; and 3$) \mathrm{TC}(2,1,5)$ codes. The curve marked by triangles represents the performance of the TC codes, when allocating the parity bits to the higher integrity protection class I and the data bits to the lower integrity protection class II. On the other hand, the performance curve marked by diamonds indicates the allocation of data bits to protection class I, while the parity bits are assigned to protection class II.

In Fig. 15(a), we can see that at low $E_{b} / N_{0}$ values, the performance of the $\mathrm{TC}(2,1,3)$ code when allocating the parity bits to protection class I is worse than allocating the data bits to protection class I. However, for $E_{b} / N_{0}$ values in excess of about $4 \mathrm{~dB}$, the situation is reversed. At a BER of $10^{-5}$, there is a performance gain of about $1 \mathrm{~dB}$ when using the 


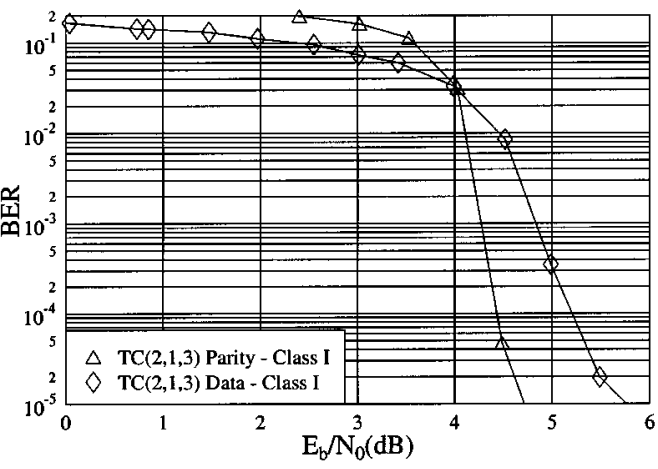

(a)

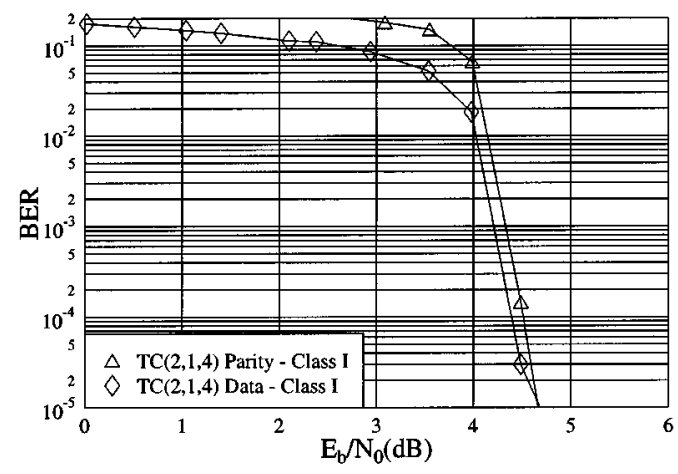

(b)

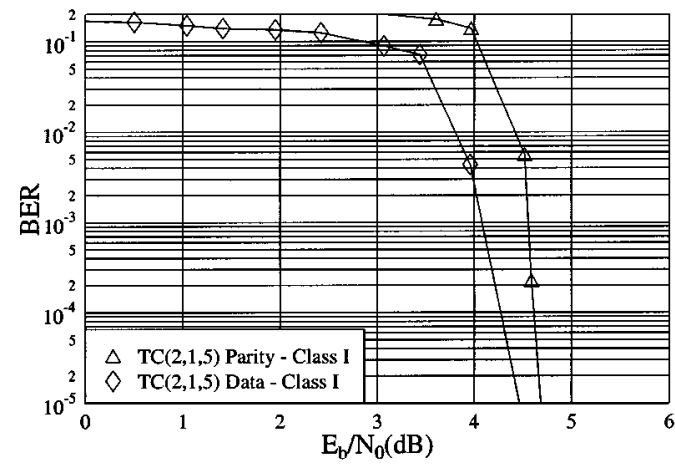

(c)

Fig. 15. Performance comparison of various data and parity bit allocation schemes for the (a) $\mathrm{TC}(2,1,3)$, (b) $\mathrm{TC}(2,1,4)$, and (c) $\mathrm{TC}(2,1,5)$ codes, where the parameters are shown in Table 3 . All simulation results were obtained upon employing the space-time code $\mathbf{G}_{2}$ using one receiver and 16QAM over uncorrelated Rayleigh fading channels at an effective throughput of 2 BPS.

$\mathrm{TC}(2,1,3)$ arrangement with the parity bits allocated to protection class I. We surmise that by protecting the parity bits better, we render the $\mathrm{TC}(2,1,3)$ code more powerful. This is related to the observation that stronger channel codes typically perform worse than weaker codes at low $E_{b} / N_{0}$ values, but naturally, these stronger codes outperform their less powerful counterparts at high $E_{b} / N_{0}$ values. This is justified by the fact that the more powerful and, hence, higher minimum distance codes are expected to inflict a higher number decoding errors in case of an erroneous decision, which frequently occur under $E_{b} / N_{0}$ conditions. This is further justified in Fig. 16. Here, we show the performance of hard-decision algebraic decoding of the $\operatorname{BCH}(7,4), \operatorname{BCH}(63,36)$, and $\mathrm{BCH}(127,71)$ codes using BPSK over additive white
BER against $E_{b} / N_{0}$

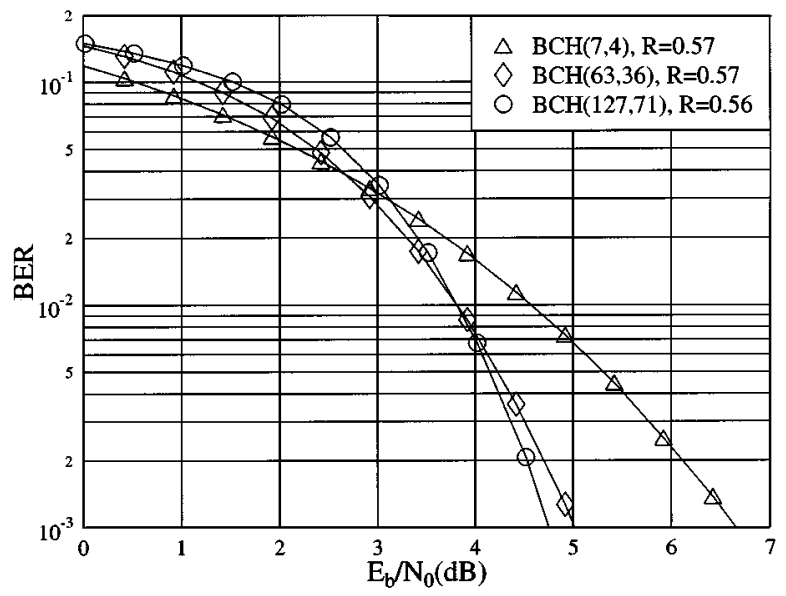

Fig. 16. Performance comparison of hard decision algebraic decoding of different $\mathrm{BCH}$ codes having approximately the same code rate of $R=0.57$, using BPSK over AWGN channels.

gaussian noise (AWGN) channels. All BCH codes characterized in Fig. 16 have approximately the same code rate, which is $R=0.57$. From Fig. 16, we can see that at a BER of $10^{-3}$, the performance of the $\mathrm{BCH}$ codes improves with an increasing codeword length $n$. However, at a high BER or low $E_{b} / N_{0}$ value, we can see that the performance of the $\operatorname{BCH}(7,4)$ code is better than that of the $\operatorname{BCH}(63,36)$ and $\mathrm{BCH}(127,71)$ codes, which are stronger channel codes, i.e., because stronger codes have many codewords having a large free distance. At low signal-to-noise ratios, we have bad channel conditions and, hence, the channel might corrupt even those codewords having a large free distance. Once they are corrupted, they produce many erroneous information bits, a phenomenon which results in a poorer BER performance.

In Fig. 15(b), we show the performance of the $\mathrm{TC}(2,1,4)$ code using the same data and parity bit allocation as in Fig. 15(a). The figure clearly shows that the $\mathrm{TC}(2,1,4)$ scheme exhibits a better performance for $E_{b} / N_{0}$ values below about $4.7 \mathrm{~dB}$, if the data bits are more strongly protected than the parity bits. It is also seen from the figure that the situation is reversed for $E_{b} / N_{0}$ values above this point. This phenomenon is different from the behavior of the $\mathrm{TC}(2,1,3)$ scheme, since the crossover point of the curves occurs at a significantly lower BER. The same situation can be observed for the $\mathrm{BCH}$ codes characterized in Fig. 16, where we can see that the performance curve of the $\mathrm{BCH}(127,71)$ code crosses the performance curve of the $\operatorname{BCH}(63,36)$ scheme at $E_{b} / N_{0} \approx 4 \mathrm{~dB}$. This value is lower than the crossover point of the performance curves of the $\operatorname{BCH}(63,36)$ and $\operatorname{BCH}(7,4)$ codes. Hence, the trend is that the crossover point of stronger codes is shifted to right of the figure. Hence, the crossover point of the performance curves of stronger codes will occur at lower BERs and shifted to the right on the $E_{b} / N_{0}$ scale. From the above argument, we can speculate also in the context of TC codes that since the $\mathrm{TC}(2,1,4)$ scheme is a stronger code than the $\mathrm{TC}(2,1,3)$ arrangement, the crossover point of the associated performance curves for $\mathrm{TC}(2,1,4)$ is at a lower 
BER than that of the $\mathrm{TC}(2,1,3)$ code and appears to be shifted to right on the $E_{b} / N_{0}$ scale.

Let us now consider the same performance curves in the context of the significantly stronger $\mathrm{TC}(2,1,5)$ code in Fig. 15(c). The figure clearly shows that better performance is yielded in the observed range, when the data bits are more strongly protected. Unlike in Fig. 15(a) and (b), there is no visible crossover point in Fig. 15(c). However, judging from the gradient of both curves, if we were to extrapolate the curves in Fig. 15 (c), they might cross at BER $\approx 10^{-6}$. The issue of data and parity bit mapping to multilevel modulation schemes was also addressed by Goff et al. [78]. However, the authors only investigated the performance of the $\mathrm{TC}(2,1,5)$ code and stated that better performance is achieved by protecting the data bits more strongly. Additionally, we note here that the situation was reversed for the $\mathrm{TC}(2,1,3)$ code, where better performance was achieved by protecting the parity bits more strongly.

Hence, from the three subfigures of Fig. 15, we can draw the following conclusions for the mapping of the data and parity bits to the different protection classes of the modulated symbol. For weaker half-rate turbo codes, such as the $\mathrm{TC}(2,1,3)$ arrangement, it is better to protect the parity bits more strongly. On the other hand, for stronger half-rate turbo codes, such as the $\mathrm{TC}(2,1,4)$ and $\mathrm{TC}(2,1,5)$ schemes, better performance is achieved by protecting the data bits more strongly. From our simulation results, we found that the same scenario also applies to turbo codes having code rates lower or higher than half rates, as shown in Table 5. Based on these facts, we continue our investigations into the effect of interleavers in an effort to achieve an improved performance.

2) Turbo Convolutional Codes-Interleaver Effects: In Fig. 7, we have seen that a bit-based channel interleaver is employed for the CC, TC, and TBCH codes. Since our performance results are obtained over uncorrelated Rayleigh fading channels, the purpose of the bit-based interleaver is to disperse bursts of channel errors within a modulated symbol, when it experiences a deep fade. This is vital for TC codes because according to the turbo code structure proposed by Berrou et al. in [38] and [39], at the output of the turbo encoder, a data bit is followed by the parity bits generated for its protection against errors. Therefore, in multilevel modulation schemes a particular modulated symbol could consist of the data bit and its corresponding parity bits generated for its protection. If the symbol experiences a deep fade, the demodulator would provide low-reliability values for both the data bit and the associated parity bits. In conjunction with low-reliability information, the turbo decoder may fail to correct errors induced by the channel. However, we can separate the data bit from the parity bits generated for its protection into different modulation symbols. By doing so, there is a better chance that the demodulator can provide high-reliability parity bits, which are represented by another modulation symbol, even if the data bit experienced a deep fade and vice versa. This will assist the turbo decoder in correcting errors.

More explicitly, the random interleaver shown in Fig. 7 has two different effects on the binary channel codes, namely:

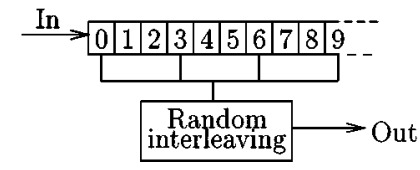

Fig. 17. Random-separation-based interleaving.

1) it separates the data bit from the parity bits generated for its protection into different modulated symbols;

2) it randomly maps the data and parity bits into different protection classes in multilevel modulation schemes.

The first-level effect of the random interleaver is to improve the performance of the binary channel codecs. In contrast, the second-level effect may have a negative impact on the performance of the channel codecs because the data and parity bits are randomly mapped to the different protection classes rather than assigning the more vulnerable bits consistently to the higher integrity protection class.

In order to eliminate the potentially detrimental second effect of the random interleaver, we propose to invoke a so-called random-separation-based interleaver. Explicitly, Fig. 17 shows an example of the random-separation-based interleaving employed. The objective of random-separation-based interleaving is to randomly interleave the bits within the same protection class of the multilevel modulated symbols. If 8PSK modulation is used, 3 BPS are transmitted. Hence, for every 3-bit spaced position, the bits will be randomly interleaved. For example, in Fig. 17, we randomly interleaved the bit positions $0,3,6,9, \ldots$. Similarly, bit positions $1,4,7, \ldots$ and $2,5,8, \ldots$ will be randomly interleaved as well.

In Fig. 18, we investigate the effects of both a random interleaver and those of a random-separation-based interleaver on the performance of the $\mathrm{TC}(2,1,3)$ code. The encoding parameters of the $\mathrm{TC}(2,1,3)$ code are shown in Table 3 . The simulation results were obtained in conjunction with the space-time code $\mathbf{G}_{2}$ using one receiver and 16QAM over uncorrelated Rayleigh fading channels. The performance curves marked by the triangles and diamonds were obtained by protecting the parity bits and data bits more strongly, respectively. Recall that the same performance curves were also shown in Fig. 15(a).

As mentioned earlier, the random interleaver has two different effects on the performance of binary channel codes. It randomly maps the data and parity bits into different protection classes, which might have a negative impact on the performance of the channel codecs. Additionally, it may separate the data bits and parity bits generated for their protection into different modulated symbols, which on the other hand, may improve the performance. In Fig. 18, the random-interleaver-based performance curve is marked by the hearts, which is similar to that of the $\mathrm{TC}(2,1,3)$-coded scheme protecting the parity bits more strongly. This suggests that the above-mentioned positive effect of the random interleaver is more pronounced than the negative effect in the context of the $\mathrm{TC}(2,1,3)$-coded scheme. On the other hand, based on the evidence of Fig. 15(a), the random-separation-based interleaver was ultimately applied in conjunction with the allo- 
BER against $\mathrm{E}_{\mathrm{b}} / \mathrm{N}_{0}$

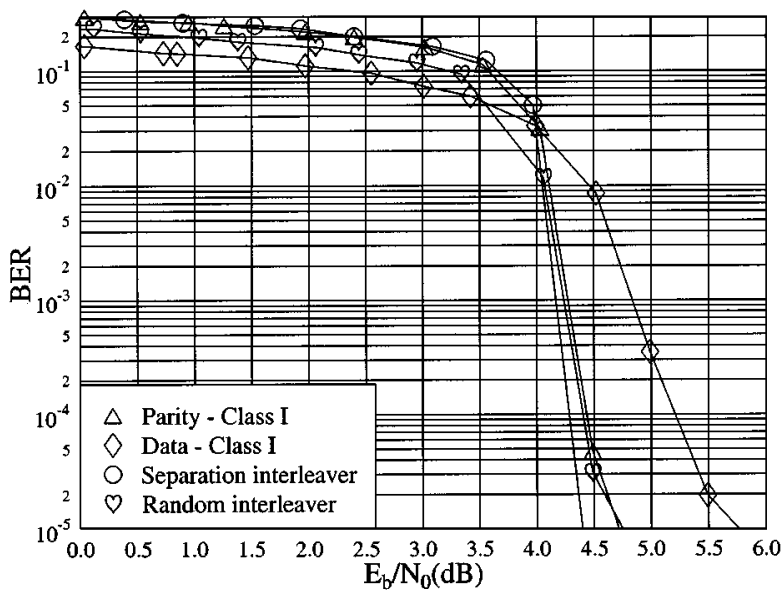

Fig. 18. Performance comparison between different bit-to-symbol mapping methods for the $\mathrm{TC}(2,1,3)$ code in conjunction with the space-time code $\mathbf{G}_{2}$ using one receiver and 16QAM over uncorrelated Rayleigh fading channels at an effective throughput of 2 BPS. Encoding parameters of the $\mathrm{TC}(2,1,3)$ code are shown in Table 3 .

cation of the parity bits, rather than the data bits into protection class I. The interleaver randomly interleaves the coded bits within the same protection class of a block of transmitted symbols. Therefore, the parity bits remain more protected compared to the data bits and yet they have been randomly interleaved within the set of parity bits. In Fig. 18, the performance of the random-separation-based interleaver, marked by circles, is about $0.5 \mathrm{~dB}$ better than that of the $\mathrm{TC}(2,1,3)$-coded scheme with the parity bits allocated to protection class I.

Similarly to Fig. 18, Figs. 19 and 20 show the performances of the $\mathrm{TC}(2,1,4)$ and $\mathrm{TC}(2,1,5)$ codes, respectively, using different bit-to-symbol mapping methods. All simulation results were obtained in conjunction with the space-time code $\mathbf{G}_{2}$ using one receiver and 16QAM over uncorrelated Rayleigh fading channels. The encoding parameters of the $\mathrm{TC}(2,1,4)$ and $\mathrm{TC}(2,1,5)$ codes are shown in Table 3. Unlike Fig. 18, the random-separation-based interleaver was applied in conjunction with the allocation of the data bits, rather than the parity bits to protection class I. It can be seen from Figs. 19 and 20 that the performance of the random-interleaver and random-separation-based interleaver is similar. This again suggests that the above-mentioned positive effect yielded by the random-based interleaver is more pronounced than its detrimental effect in the context of both the $\mathrm{TC}(2,1,4)$ and $\mathrm{TC}(2,1,5)$ schemes.

In conclusion, the simulation results presented in this section demonstrate that at a BER of $10^{-\tilde{5}}$, the half-rate turbo codes using a random-separation-based interleaver attain the best performance, albeit for certain schemes only by a small margin. Therefore, in our forthcoming performance comparisons, we will be employing the random-separation-based interleaver in conjunction with the various TC codes.

3) TBCH Codes: Fig. 21 characterizes the performance of the $\operatorname{TBCH}(32,26)$ code in conjunction with different bit-to-symbol mappings to the two protection classes of
BER against $\mathrm{E}_{\mathrm{b}} / \mathrm{N}_{0}$

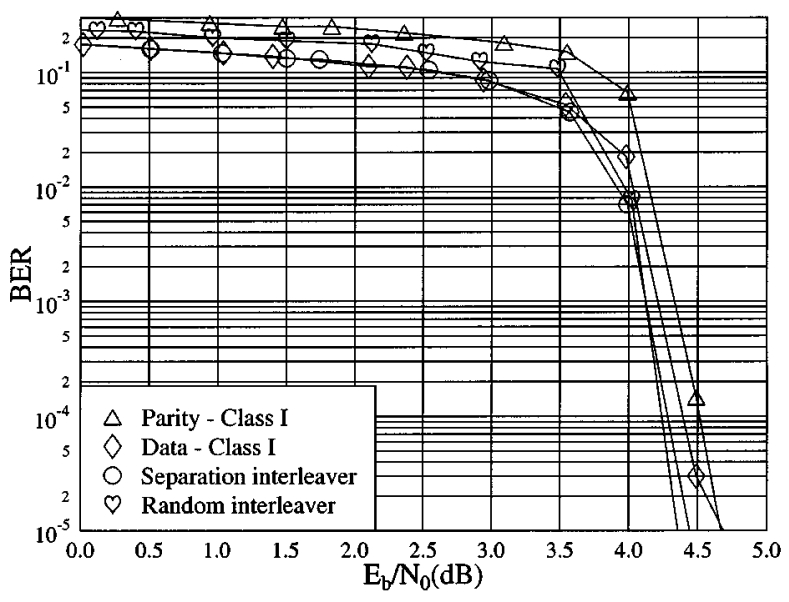

Fig. 19. Performance comparison between different bit-to-symbol mapping methods for the $\mathrm{TC}(2,1,4)$ code in conjunction with the space-time code $\mathbf{G}_{2}$ using one receiver and 16QAM over uncorrelated Rayleigh fading channels at an effective throughput of 2 BPS. Encoding parameters of the $\mathrm{TC}(2,1,4)$ code are shown in Table 3 .

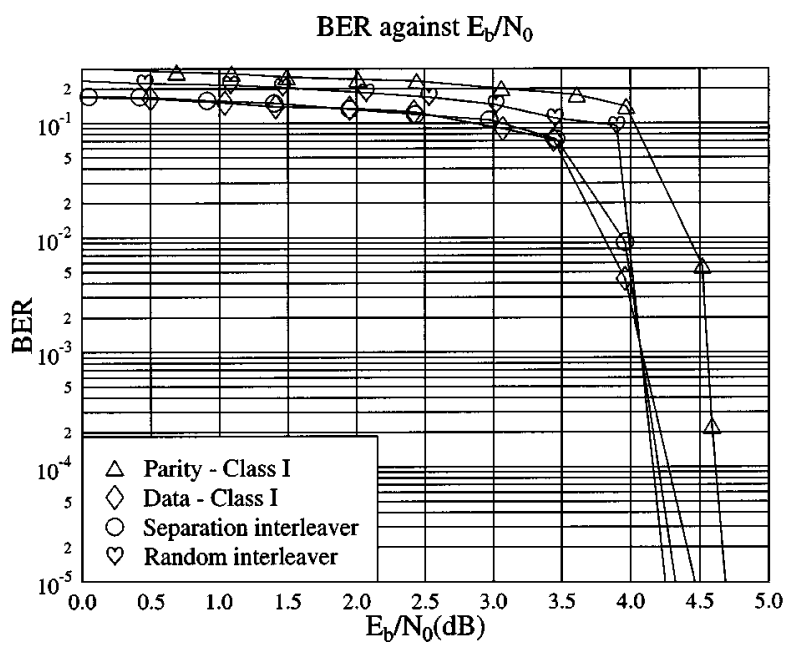

Fig. 20. Performance comparison between different bit-to-symbol mapping methods for the $\mathrm{TC}(2,1,5)$ code in conjunction with the space-time code $\mathbf{G}_{2}$ using one receiver and 16QAM over uncorrelated Rayleigh fading channels at an effective throughput of 2 BPS. Encoding parameters of the $\mathrm{TC}(2,1,5)$ code are shown in Table 3.

8PSK. All simulation results were obtained with the aid of the space-time code $\mathbf{G}_{2}$ using one receiver and 8PSK over uncorrelated Rayleigh fading channels. Again, the encoding parameters of the $\operatorname{TBCH}(32,26)$ code are shown in Tables 3 and 5. The $\operatorname{TBCH}(32,26)$ code was chosen for our investigations because the parity bits of the constituent encoders were not punctured and hence this resulted in a code rate of $R \approx 2 / 3$. Roughly speaking, for every two data bits, there is one parity bit. Similarly to 16QAM, in the Gray-mapping-assisted 8PSK constellation, there are also two protection classes, depending on the bit position in the 3-bit symbols. In the three bits of the 8PSK constellation, two of the bit positions are more protected than the remaining bit. In Fig. 21, we portray the performance of the $\operatorname{TBCH}(32,26)$ scheme for four different bit-to-symbol 


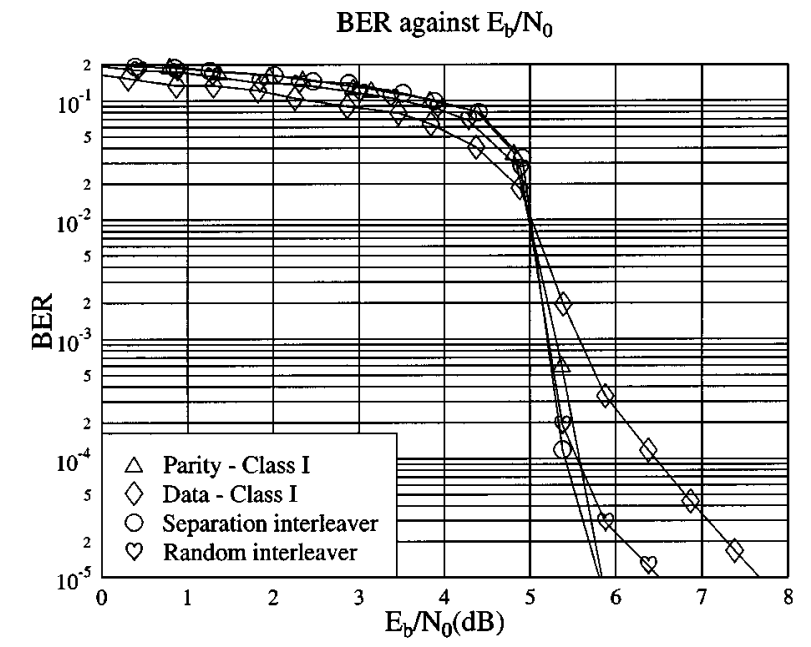

Fig. 21. Performance comparison between different bit-to-symbol mapping methods for the $\operatorname{TBCH}(32,26)$ code in conjunction with the space-time code $\mathbf{G}_{2}$ using one receiver and 8PSK over uncorrelated Rayleigh fading channels at an effective throughput of 2 BPS. Encoding parameters of the $\operatorname{TBCH}(32,26)$ code are shown in Tables 3 and 5 .

mapping methods. First, one data bit and one parity bit was mapped to the two better protected 8PSK bit positions. The corresponding BER curve is marked by the triangles in Fig. 21. According to the second method, the data bits were mapped to the two better protected bit positions of the 8PSK symbol. This scenario is marked by the diamonds in Fig. 21. As we can see from the figure, the first mapping method yields a substantial $E_{b} / N_{0}$ gain of $1.5 \mathrm{~dB}$ at a BER of $10^{-5}$ over the second method. By applying the random-separation-based interleaver of Fig. 17, while still better protecting one of the data bits and the parity bit than the remaining data bits, we disperse the bursty bit errors associated with a transmitted symbol over several $\mathrm{BCH}$ codewords of the TBCH code. As shown in Fig. 21, the performance curve marked by the circles shows a slight improvement compared to the above-mentioned first method, although the difference is marginal. Finally, we show the performance of applying random interleaving, which randomly distributes the data and parity bits between the two 8PSK protection classes. It can be seen that the associated performance is worse than that of the first bit-to-symbol mapping method.

Fig. 21 shows that it is better to protect the parity bits more strongly for the $\operatorname{TBCH}(32,26)$ code and a slight further improvement can be achieved by applying a random-separation-based interleaver. More simulation results were obtained in conjunction with the other TBCH codes shown in Tables 3 and 5 with the aid of the space-time code $\mathbf{G}_{2}$ and 64QAM over uncorrelated Rayleigh fading channels. From the simulation results we have found that all $\mathrm{TBCH}$ codes shown in Tables 3 and 5 perform better, if the parity bits are more protected. In general, a slight further improvement can be obtained for TBCH codes, when a random-separation-based interleaver is applied. A possible explanation is that the component encoders of the $\mathrm{TBCH}$ codes are $\mathrm{BCH}$ encoders, where a block of parity bits is generated by a block of data bits. Hence, every parity bit has an influence on the

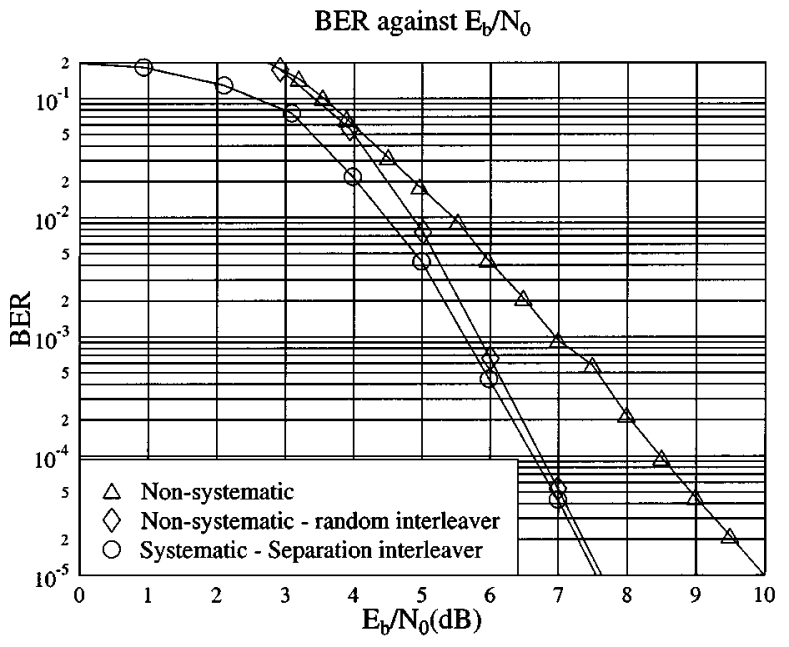

Fig. 22. Performance comparison between the systematic and nonsystematic half-rate $\mathrm{CC}(2,1,9)$ code in conjunction with the space-time code $\mathbf{G}_{2}$ and 16QAM over uncorrelated Rayleigh fading channels at a throughput of 2 BPS. Encoding parameters of the $\mathrm{CC}(2,1,9)$ code are shown in Tables 3 and 4 .

whole codeword. Moreover, we used high-rate TBCH codes and, hence, there are more data bits compared to the parity bits. Hence, in our forthcoming TBCH comparisons, we will use bit-to-symbol mappers protecting the parity bits better.

4) Convolutional Codes: Let us now investigate the space-time code $\mathbf{G}_{2}$ in conjunction with the half-rate $\mathrm{CC}$ $\mathrm{CC}(2,1,9)$ proposed for UMTS. The $\mathrm{CC}(2,1,9)$ code is a nonsystematic nonrecursive $\mathrm{CC}$, where the original information bits cannot be explicitly recognized in the encoded sequence. Its associated performance curve is shown in Fig. 22 marked by the triangles. A random interleaver is applied in order to disperse the bursty channel errors and the associated performance curve is marked by the diamonds in Fig. 22. At a BER of $10^{-5}$, there is a performance gain of 2.5 $\mathrm{dB}$ if the random interleaver is applied. As a further scheme, we implemented a systematic $\mathrm{CC}(2,1,9)$ code, which was obtained using a recursive CC [72], [100]. Hence, in this scenario, we have explicitly separable data bits and parity bits. In Fig. 22, the performance curve marked by the circles is obtained by mapping the data bits-rather than the parity bits - of the systematic $\mathrm{CC}(2,1,9)$ code to protection class I of the associated 16QAM scheme in conjunction with the random-separation-based interleaver of Fig. 17. Explicitly, the data bits rather than the parity bits of the systematic $\mathrm{CC}(2,1,9)$ code are mapped to protection class I of the associated 16QAM scheme, since for this powerful code, similar performance trends are expected to those experienced for the $\mathrm{CC}(2,1,5)$. One can see that there is only a marginal performance improvement over the nonsystematic $\mathrm{CC}(2,1,9)$ code using the random interleaver.

\section{Performance Comparison of Various Channel Codecs Using the $\mathbf{G}_{2}$ Space-Time Code and Multilevel Modulation}

In this section, we compare the $\mathbf{G}_{2}$ space-time-coded performance of all channel codecs summarized in Table 3. In order to avoid having an excessive number of curves in one figure, only one channel codec will be characterized 
BER against $\mathrm{E}_{\mathrm{b}} / \mathrm{N}_{0}$

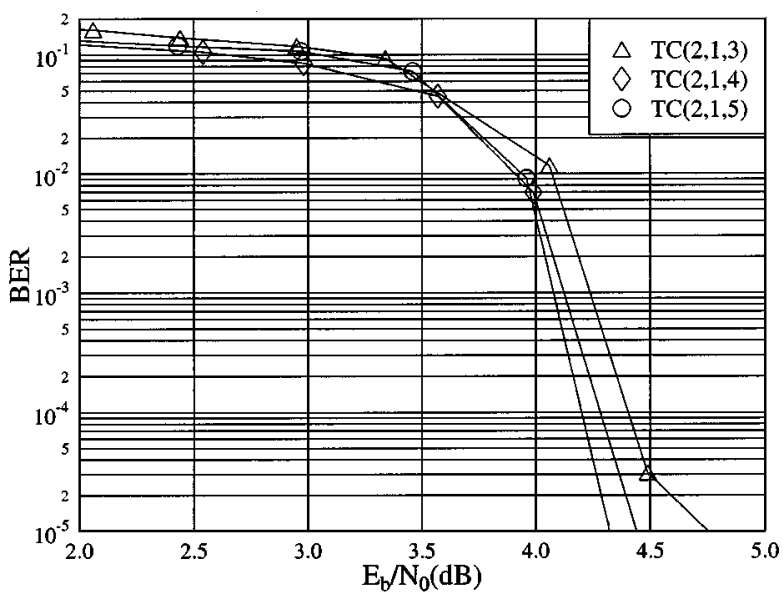

Fig. 23. Performance comparison between the half-rate codes $\mathrm{TC}(2,1,3)$, $\mathrm{TC}(2,1,4)$, and $\mathrm{TC}(2,1,5)$, where the encoding parameters are shown in Tables 3 and 5. All simulation results were obtained with the aid of the space-time code $\mathbf{G}_{2}$ using 16QAM over uncorrelated Rayleigh fading channels and the throughput was 2 BPS.

from each group of the $\mathrm{CC}$, TC, TBCH, TCM, and TTCM schemes. The choice of the channel codec considered depends on its performance, complexity, and code rate. Unless otherwise stated, all channel codecs are concatenated with the space-time code $\mathbf{G}_{2}$ using one receiver. All comparisons are carried out on the basis of the same BPS throughput over uncorrelated Rayleigh fading channels. Let us now briefly discuss in the forthcoming sections how each channel codec is selected from the codec families considered.

1) Comparison of Turbo Convolutional Codes: In Fig. 23, we compare the performance of the half-rate turbo codes $\mathrm{TC}(2,1,3)$, $\mathrm{TC}(2,1,4)$, and $\mathrm{TC}(2,1,5)$, where the encoding parameters are shown in Tables 3 and 5. The simulation results were obtained with the aid of the space-time code $\mathbf{G}_{2}$ using 16QAM over uncorrelated Rayleigh fading channels. The three performance curves in the figure are the best performance curves chosen from Figs. 20, 19, and 18 for the half-rate codes $\mathrm{TC}(2,1,5), \mathrm{TC}(2,1,4)$, and $\mathrm{TC}(2,1,3)$, respectively. It can be seen from the figure that the performance of the turbo codes improves, when we increase the constraint length of the component codes from three to five. However, this performance gain is obtained at the cost of a higher decoding complexity. At a BER of $10^{-5}$, the $\mathrm{TC}(2,1,4)$ code has an $E_{b} / N_{0}$ improvement of approximately $0.25 \mathrm{~dB}$ over the $\mathrm{TC}(2,1,3)$ scheme at a penalty of twice the complexity. However, at the cost of the same complexity increment over that of the $\mathrm{TC}(2,1,4)$ arrangement, the $\mathrm{TC}(2,1,5)$ scheme only achieves a marginal performance gain of $0.1 \mathrm{~dB}$ at $\mathrm{BER}=10^{-5}$. Therefore, in our following investigations, only the $\mathrm{TC}(2,1,4)$ scheme will be characterized as it exhibits a significant coding gain at a moderate complexity. Furthermore, the $\mathrm{TC}(2,1,4)$ code has been adopted by the $3 \mathrm{G}$ UTRA mobile communication system [72].

2) Comparison of Different Rate $\mathrm{TC}(2,1,4)$ Codes: In their seminal paper on turbo coding [38], [39], Berrou et al.

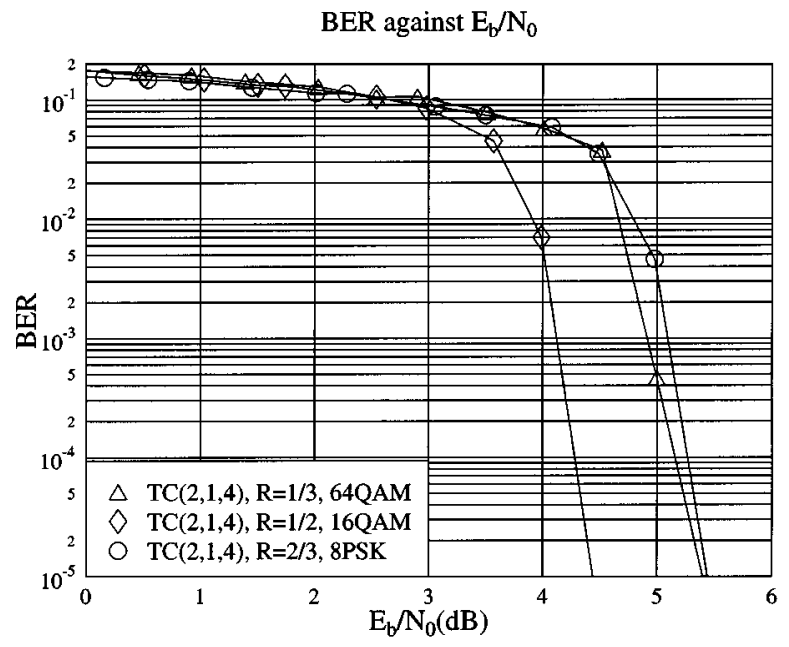

Fig. 24. Performance of the $\mathrm{TC}(2,1,4)$ code using coding rates of $1 / 3,1 / 2$, and $2 / 3$, where the associated encoding parameters are shown in Tables 3 and 5. All simulation results were obtained with the aid of the space-time code $\mathbf{G}_{2}$ at an effective throughput of 2 BPS over uncorrelated Rayleigh fading channels.

applied alternate puncturing of the parity bits. This results in half-rate turbo codes. Additionally, a range of different puncturing patterns can be applied, which results in different code rates [87]. In Fig. 24, we portray the performance of the punctured $\mathrm{TC}(2,1,4)$ code having coding rates of $1 / 3,1 / 2$, and $2 / 3$. The associated coding parameters are shown in Tables 3 and 5. Suitable multilevel modulation schemes are chosen so that all systems have the same effective throughput of 2 BPS. Explicitly, 64QAM, 16QAM, and 8PSK are used. All simulation results were obtained with the aid of the space-time code $\mathbf{G}_{2}$ over uncorrelated Rayleigh fading channels. As expected, from Fig. 24, we can clearly see that the best performance is achieved by the half-rate $\mathrm{TC}(2,1,4)$ scheme. At a BER of $10^{-5}$, the half-rate $\mathrm{TC}(2,1,4)$ code achieved a performance gain of approximately $1 \mathrm{~dB}$ over the third-rate and the two-third-rate $\mathrm{TC}(2,1,4)$ codes. Even though the third-rate $\mathrm{TC}(2,1,4)$ code has a higher amount of redundancy than the half-rate $\mathrm{TC}(2,1,4)$ scheme, its performance is worse than that of the half-rate $\mathrm{TC}(2,1,4)$ arrangement. We speculate that this is because the constellation points in 64 QAM are more densely packed than those of 16QAM. Therefore, they are more prone to errors and hence the extra coding power of the third-rate $\mathrm{TC}(2,1,4)$ code is insufficient to correct the extra errors. This results in a poorer performance. On the other hand, there are fewer errors induced by 8PSK, but the two-third-rate $\mathrm{TC}(2,1,4)$ code is a weak code due to the puncturing of the parity bits. Again, this results in an inferior performance.

In Fig. 25, we show the performance of the $\mathrm{TC}(2,1,4)$ code at coding rates of $1 / 2$ and $3 / 4$. The associated coding parameters are shown in Tables 3 and 5. Again, suitable modulation schemes were chosen so that both systems have the same effective throughput, namely, 3 BPS. All simulation results were obtained with the aid of the space-time code $\mathbf{G}_{2}$ over uncorrelated Rayleigh fading channels. As compared to Fig. 24, the throughputs of the systems in Fig. 25 have been increased from 2 to 3 BPS. In order to maintain 


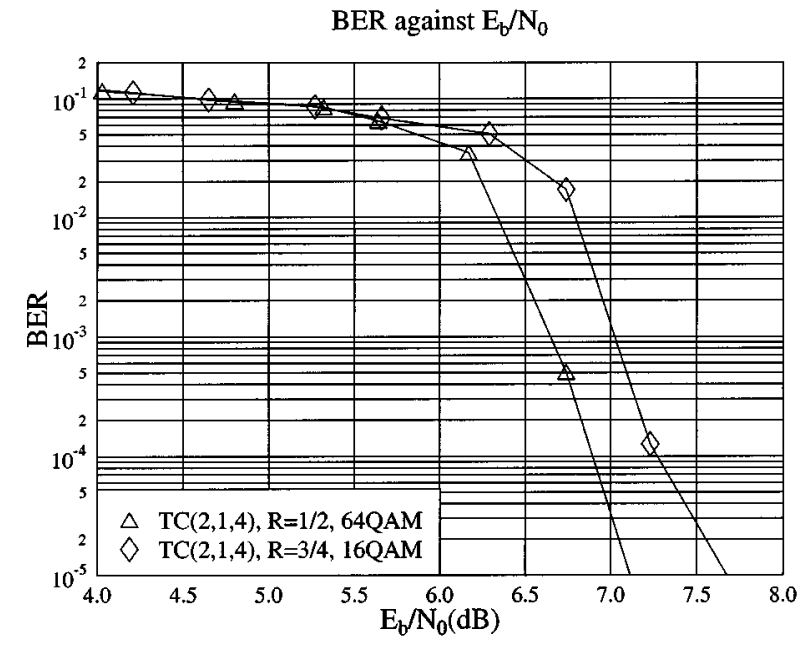

Fig. 25. Performance of the punctured $\mathrm{TC}(2,1,4)$ code at coding rates of $1 / 2$ and $3 / 4$, where the associated parameters are shown in Tables 3 and 5. All simulation results were obtained with the aid of the space-time code $\mathbf{G}_{2}$ at an effective throughput of 3 BPS over uncorrelated Rayleigh fading channels.

a high BPS throughput, 64QAM was employed in conjunction with the half-rate $\mathrm{TC}(2,1,4)$ code. We can see from the figure that the performance gain of the half-rate $\mathrm{TC}(2,1,4)$ code over the three-quarter-rate $\mathrm{TC}(2,1,4)$ code has been reduced to only $0.5 \mathrm{~dB}$, as compared to $1 \mathrm{~dB}$ over the twothird-rate $\mathrm{TC}(2,1,4)$ code characterized in Fig. 24. Moreover, the three-quarter-rate $\mathrm{TC}(2,1,4)$ code is weaker than the two-third-rate $\mathrm{TC}(2,1,4)$ code, since fewer parity bits are transmitted over the channel. Based on the fact that the performance gain of the half-rate $\mathrm{TC}(2,1,4)$ code has been reduced, we surmise that high-rate turbo codes will outperform the half-rate $\mathrm{TC}(2,1,4)$ code, if the throughput of the system is increased to 4 BPS or even further, since half-rate codes would require a vulnerable high-order modulation constellation for maintaining a throughput of 4 BPS.

From Figs. 24 and 25, we can see that the best performance is achieved by the half-rate $\mathrm{TC}(2,1,4)$ code for an effective throughput of 2 and 3 BPS. However, we are also interested in the system's performance at higher effective BPS throughputs. Hence, during our later discourse in Section VI-C6, the performance of high-rate TC and TBCH codes will be studied for throughput values in excess of 5 BPS.

3) Convolutional Codes: In Fig. 26, we compare the performances of the $\mathbf{G}_{2}$ space-time-coded nonrecursive half-rate $\mathrm{CCs} \mathrm{CC}(2,1,5), \mathrm{CC}(2,1,7)$, and $\mathrm{CC}(2,1,9)$. These schemes were standardized in the GSM [72], [107], DVB [65], and the 3G UTRA systems [1], [72], [109], respectively. The associated coding parameters are shown in Tables 3 and 4. All simulation results were obtained with the aid of the space-time code $\mathbf{G}_{2}$ using QPSK over uncorrelated Rayleigh fading channels. We can see from the figure that at a BER of $10^{-5}$, the performance of the nonrecursive CCs improves by approximately $1 \mathrm{~dB}$, if the complexity is increased by a factor of $2^{2}=4$. However, the extra performance gain attainable becomes smaller, as the affordable complexity further increases. In our forthcoming
BER against $\mathrm{E}_{\mathrm{b}} / \mathrm{N}_{0}$

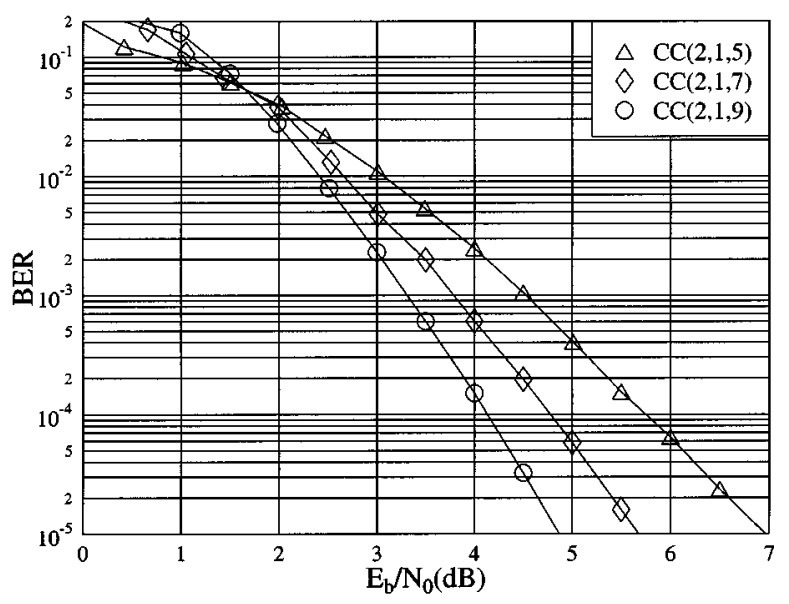

Fig. 26. Performance comparison between the nonrecursive half-rate $\mathrm{CCs} \mathrm{CC}(2,1,5), \mathrm{CC}(2,1,7)$, and $\mathrm{CC}(2,1,9)$, where the coding parameters are shown in Tables 3 and 4 . All simulation results were obtained with the aid of the space-time code $\mathbf{G}_{2}$ using QPSK over uncorrelated Rayleigh fading channels. Effective throughput is 1 BPS.

channel code comparisons, only the $\mathrm{CC}(2,1,9)$ code will be used, since it has the best performance among the above three schemes and it has a comparable complexity to that of the TC codes studied. Moreover, the $\mathrm{CC}(2,1,9)$ code is also proposed for the 3G UTRA mobile communication system [72].

4) $\mathbf{G}_{2}$-Coded Channel-Codec Comparison-Throughput of 2 BPS: Having narrowed down the choice of the $\mathbf{G}_{2}$ space-time-coded CCs and the turbo codes, we are now ready to compare the performances of the different proposed channel codecs belonging to different codec families. Our comparison is carried out on the basis of the same throughput and all channel codecs are concatenated with the space-time code $\mathbf{G}_{2}$, when transmitting over uncorrelated Rayleigh fading channels. Fig. 27 shows the performance of our channel codecs selected from the CC, TC, TBCH, TCM, and TTCM families on the basis of the same throughput of 2 BPS, regardless of their coding rates. The associated coding parameters are shown in Tables 3-5. The throughput is 2 BPS.

From Fig. 27, we can see that the half-rate $\mathrm{TC}(2,1,4)$ code outperforms the other channel codecs. At a BER of $10^{-5}$, the $\mathrm{TC}(2,1,4)$ code achieves a gain of approximately $0.5 \mathrm{~dB}$ over the TBCH $(31,21)$ scheme at a much lower complexity. At the same BER, the TC $(2,1,4)$ code also outperforms 8PSK-TTCM by approximately $1.5 \mathrm{~dB}$. The poor performance of TTCM might be partially due to using generator polynomials, which are optimum for AWGN channels [80]. However, to date, only limited research has been carried out on finding optimum generator polynomials for TTCM over fading channels [110].

In Fig. 27, we also characterize the performance of the $\mathrm{CC}(2,1,9)$ and 8PSK-TCM schemes. The figure clearly demonstrates that the invention of turbo codes invoked in our TC, TBCH, and TTCM $\mathbf{G}_{2}$-coded schemes, resulted in 
BER against $\mathrm{E}_{\mathrm{b}} / \mathrm{N}_{0}$

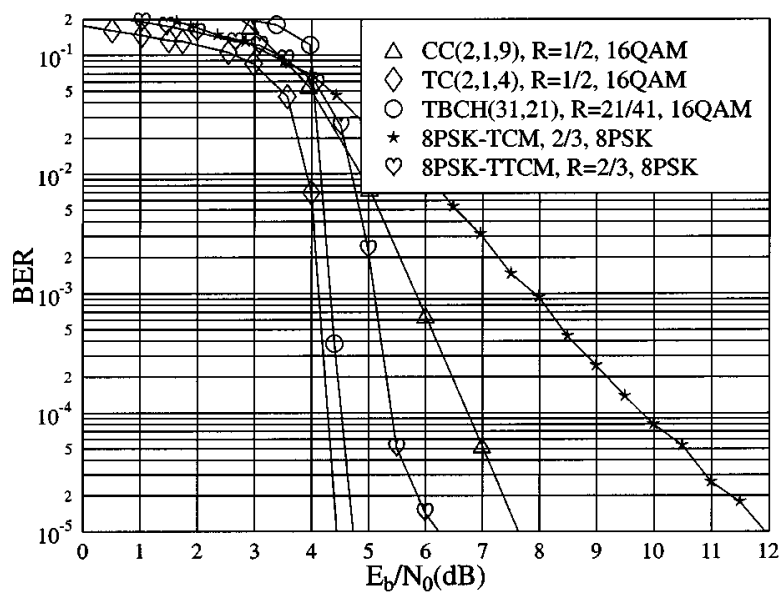

Fig. 27. Performance comparison between different $\mathrm{CC}$, TC, TBCH, TCM, and TTCM schemes, where the coding parameters are shown in Tables 3-5. All simulation results were obtained with the aid of the space-time code $\mathbf{G}_{2}$ at a throughput of 2 BPS over uncorrelated Rayleigh fading channels.

substantial improvements over the conventional $\mathbf{G}_{2}$-coded channel codecs, such as the CC and TCM schemes considered. At a BER of $10^{-5}$, the TC $(2,1,4)$ code outperforms the $\mathrm{CC}(2,1,9)$ and 8 PSK-TCM arrangements by approximately 3.0 and $7.5 \mathrm{~dB}$, respectively.

5) $\mathbf{G}_{2}$-Coded Channel Codec Comparison-Throughput of 3 BPS: In Fig. 28, we portray the performances of various channel codecs belonging to the $\mathrm{CC}, \mathrm{TC}, \mathrm{TBCH}, \mathrm{TCM}$, and TTCM codec families on the basis of a constant throughput of 3 BPS, regardless of their coding rates. The associated coding parameters are shown in Tables $3-5$. The simulation results were obtained with the aid of the space-time code $\mathbf{G}_{2}$ over uncorrelated Rayleigh fading channels.

From Fig. 28, we can infer a few interesting points. As mentioned earlier, the half-rate $\operatorname{TC}(2,1,4)$ code suffers from the effects of puncturing as we increase the throughput of the system. In order to maintain a throughput of 3 BPS, 64QAM has to be employed in the systems using the half-rate $\mathrm{TC}(2,1,4)$ code. The rather vulnerable 64QAM modulation scheme appears to overstretch the coding power of the half-rate $\mathrm{TC}(2,1,4)$ code attempting to saturate the available channel capacity. At a BER of $10^{-5}$, there is no obvious performance gain over the TBCH $(31,26) / 16 \mathrm{QAM}$ and 16QAM-TTCM schemes. Hence, we have reasons to postulate that if the throughput of the system is increased beyond 3-BPS high-rate turbo codes should be employed for improving the performance, rather than invoking a higher throughput modulation scheme.

6) Comparison of $\mathbf{G}_{2}$-Coded High-Rate TC and TBCH Codes: In the previous section, we have shown that at the BER of $10^{-5}$, the required $E_{b} / N_{0}$ is increased by about $2.5 \mathrm{~dB}$ for the half-rate turbo code $\mathrm{TC}(2,1,4)$, as the throughput of the system is increased from 2 to 3 BPS. A range of schemes having a throughput in excess of 5 BPS is characterized in Fig. 29. Specifically, the figure shows the performance of high-rate TC and TBCH codes concatenated with the space-time code $\mathbf{G}_{2}$ employing 64QAM over
BER against $\mathrm{E}_{\mathrm{b}} / \mathrm{N}_{0}$

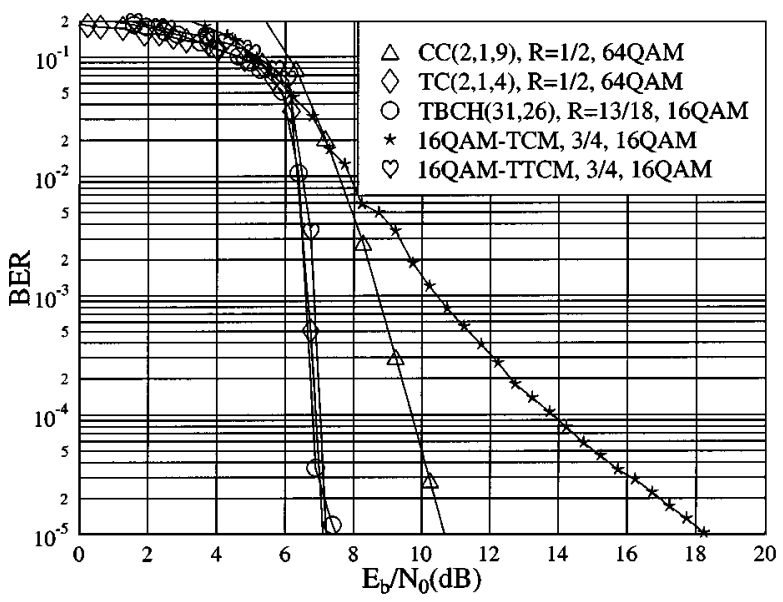

Fig. 28. Performance comparison between different CC, TC, TBCH, TCM, and TTCM schemes, where the coding parameters are shown in Tables 3-5. All simulation results were obtained with the aid of the space-time code $\mathbf{G}_{2}$ at an effective throughput of 3 BPS over uncorrelated Rayleigh fading channels.

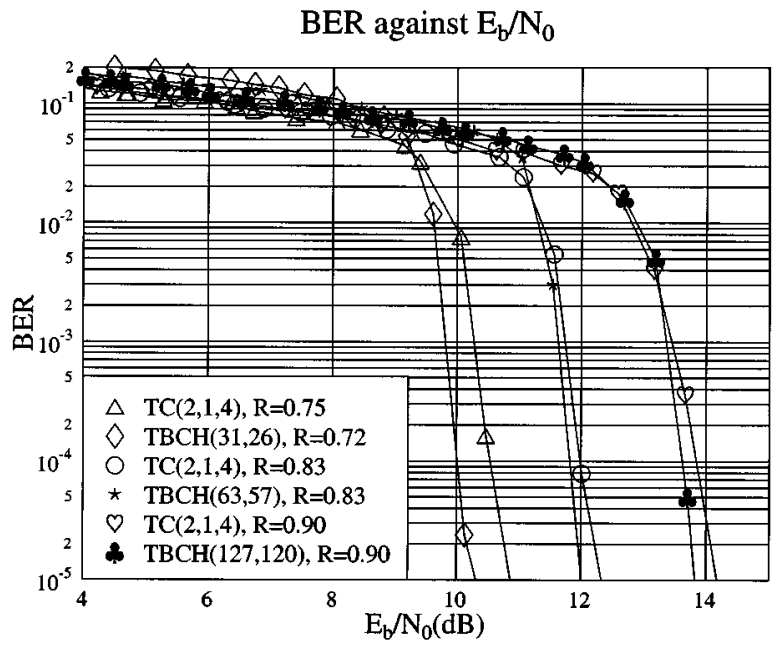

Fig. 29. Performance comparison between high-rate TC and TBCH codes concatenated with the space-time code $\mathbf{G}_{2}$ employing 64QAM over uncorrelated Rayleigh fading channels. Parameters of the TC and TBCH codes are shown in Tables 3 and 5.

uncorrelated Rayleigh fading channels. The parameters of the TC and TBCH codes used are shown in Tables 3 and 5 . The performance of half-rate turbo codes along with such a high throughput is not shown because a modulation scheme having at least 1024 constellation points would be needed, which is practically infeasible over nonstationary wireless channels. Moreover, the turbo codes often would be overloaded by errors induced by the densely packed constellation points.

In Fig. 29, we can clearly see that there is not much difference in performance terms between the high-rate $\mathrm{TC}(2,1,4)$ and TBCH codes employed, although the TBCH codes exhibit marginal gains. This gain is achieved at a cost of high decoding complexity, as evidenced by Table 6 . The slight performance improvement of the $\operatorname{TBCH}(31,26)$ code over the three-quarter-rate $\mathrm{TC}(2,1,4)$ scheme is probably due to its slightly lower code rate of $R=0.72$, compared to the 


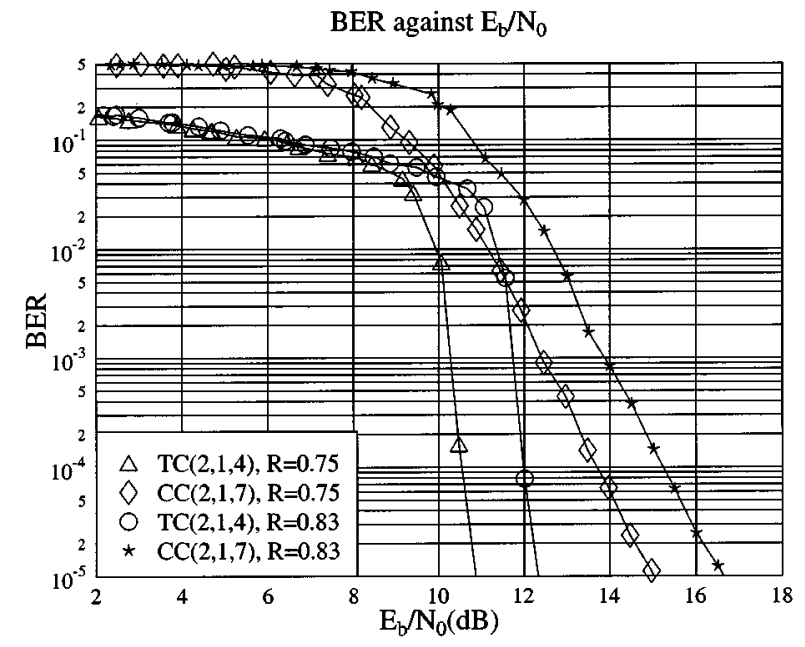

Fig. 30. Performance comparison between high-rate TCs and CCs concatenated with the space-time code $\mathbf{G}_{2}$ employing 64QAM over uncorrelated Rayleigh fading channels. Parameters of the TC and CC codes are shown in Tables 3-5.

rate of $R=0.75$ associated with the $\mathrm{TC}(2,1,4)$ code. It is important to note that all $\mathrm{BCH}$ component codes used in the TBCH codes have a minimum distance $d_{\min }$ of three. We speculate that the performance of the TBCH codes might improve, if $d_{\min }$ is increased to five. However, due to the associated complexity, we will refrain from employing $d_{\min }=5$ $\mathrm{BCH}$ component codes in the $\mathrm{TBCH}$ schemes studied.

7) Comparison of High-Rate TC and Convolutional Codes: In Fig. 30, we compare the performance of the high-rate punctured $\mathrm{TC}(2,1,4)$ and $\mathrm{CC}(2,1,7)$ codes concatenated with the space-time code $\mathbf{G}_{2}$ employing 64QAM over uncorrelated Rayleigh fading channels. The puncturing patterns employed for the $\mathrm{CC}(2,1,7)$ scheme were proposed in the DVB standard [65]. The parameters of the $\mathrm{TC}(2,1,4)$ and $\mathrm{CC}(2,1,7)$ codes are shown in Tables $3-5$. From the figure, we can see that both high-rate $\mathrm{TC}(2,1,4)$ codes outperform their equivalent rate $\mathrm{CC}(2,1,7)$ counterparts by about $2 \mathrm{~dB}$ at a BER of $10^{-5}$, while maintaining a similar estimated decoding complexity, as evidenced by Table 6 . This fact indicates that at a given tolerable complexity, better BER performance can be attained by an iterative turbo decoder. These findings motivated the investigations of our next section, where the performance of the various schemes is studied in the context of the achievable coding gain versus the estimated decoding complexity.

\section{Coding Gain Versus Complexity}

In Section V-C, we have estimated the various channel decoders' complexity based on a few simplifying assumptions. All the complexities estimated in our forthcoming discourse were calculated based on (36)-(44). Again, our performance comparison of the channel codes was made on the basis of the coding gain defined as the $E_{b} / N_{0}$ difference, expressed in decibels, at BER $=10^{-5}$ between the various channel-coded and uncoded systems having the same throughput, while using the space-time code $\mathbf{G}_{2}$.

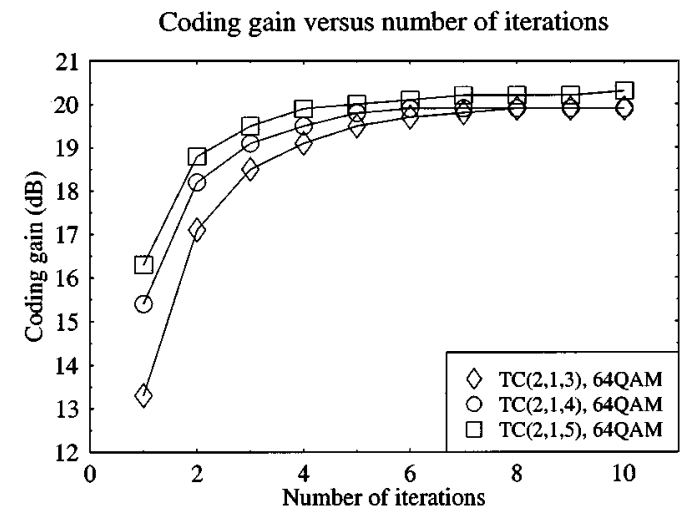

(a)

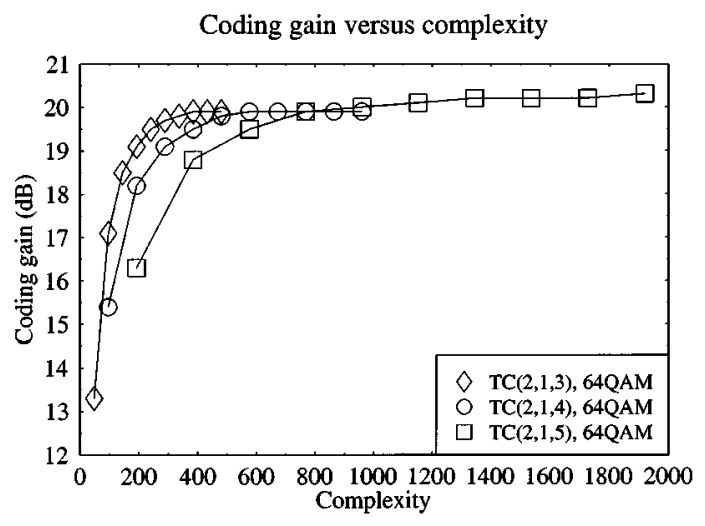

(b)

Fig. 31. Coding gain versus (a) the number iterations and (b) estimated complexity for the $\mathrm{TC}(2,1,3), \mathrm{TC}(2,1,4)$, and $\mathrm{TC}(2,1,5)$ codes, where the coding parameters are shown in Tables 3,5 , and 6 . All simulation results were obtained upon employing the space-time code $\mathbf{G}_{2}$ using one receiver and 64QAM over uncorrelated Rayleigh fading channels at an effective throughput of 3 BPS.

\section{1) Complexity Comparison of Turbo Convolutional} Codes: Fig. 31(a) shows the coding gain versus the number of iterations and Fig. 31(b) the coding gain versus estimated complexity for the $\mathrm{TC}(2,1,3), \mathrm{TC}(2,1,4)$ and $\mathrm{TC}(2,1,5)$ codes, where the coding parameters used are shown in Tables 3, 5, and 6. All simulation results were obtained upon employing the space-time code $\mathbf{G}_{2}$ using one receiver and 64QAM over uncorrelated Rayleigh fading channels at an effective throughput of 3 BPS. We can see from Fig. 31(a) that there is a substantial performance improvement of approximately 3-4 $\mathrm{dB}$ between the first and second turbo decoding iteration. However, further coding gain improvements become smaller, as the number of iterations increases. It can be seen from the figure that the performance of turbo codes does not significantly improve after eight iterations, as indicated by the rather flat coding gain curve. Fig. 31(a) also shows that as we increase the constraint length $K$ of the turbo codes from three to five, the associated performance improves.

In Fig. 31(b), the coding gains of the various turbo codes using different number of iterations are compared on the basis of their estimated complexity. This is necessary, since we have seen in Section V-C that the estimated complexity of turbo codes depends exponentially on the constraint 
Coding gain versus complexity

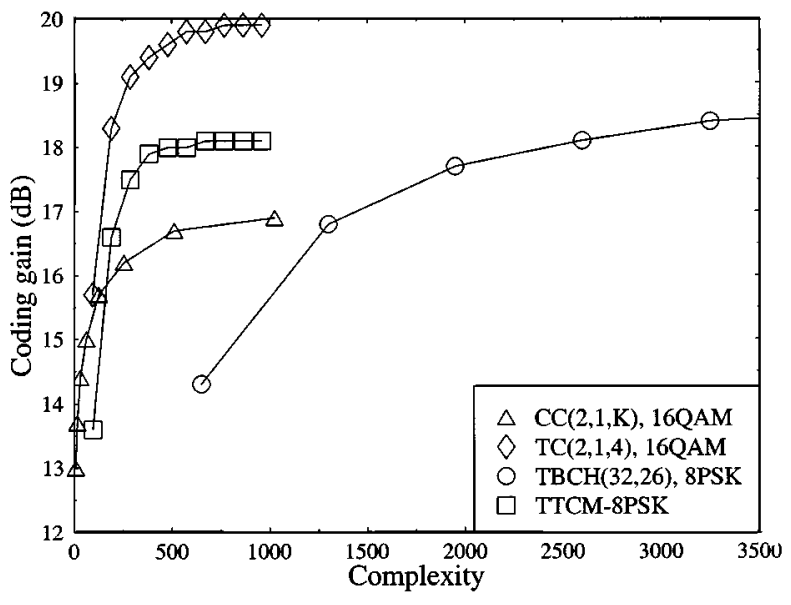

Fig. 32. Coding gain versus estimated complexity for the $\mathrm{CC}(2,1, K)$, TC $(2,1,4)$, TBCH $(32,26)$, and TTCM-8PSK, where the parameters are shown in Tables 3-6. All simulation results were obtained upon employing space-time code $\mathbf{G}_{2}$ using one receiver over uncorrelated Rayleigh fading channels at an effective throughput of 2 BPS.

length $K$, but only linearly on the number of iterations. From Fig. 31(b), we can see that the estimated complexity of the TC $(2,1,5)$ code ranges from approximately 200 to 2000 when using one to ten iterations. On the other hand, the estimated complexity of the $\mathrm{TC}(2,1,3)$ scheme ranges only from approximately 50 to 500 , requiring one to ten iterations. This clearly shows that the estimated complexity of the turbo codes is dominated by the constraint length $K$. Fig. 31(b) also shows that the coding gain curve of the $\mathrm{TC}(2,1,3)$ code saturates faster, which is demonstrated by the steep increase in coding gain as the estimated complexity increases. For achieving the same coding gain of $19 \mathrm{~dB}$, we can see that the $\mathrm{TC}(2,1,3)$ scheme requires the lowest estimated complexity. It requires two to three times higher computational power for the $\mathrm{TC}(2,1,5)$ code to achieve the above-mentioned coding gain of $19 \mathrm{~dB}$.

2) Complexity Comparison of Channel Codes: In the previous section, we compared the coding gain versus estimated complexity of the $\mathbf{G}_{2}$-coded turbo schemes $\mathrm{TC}(2,1,3)$, $\mathrm{TC}(2,1,4)$, and $\mathrm{TC}(2,1,5)$. Here, we compare the $\mathrm{TC}(2,1,4)$ arrangement that faired best amongst them to the $\mathrm{CC}(2,1,9)$ code and to the $\operatorname{TBCH}(32,26) / 8 \mathrm{PSK}$ as well as to the TTCM-8PSK arrangements, representing the other codec families studied. Specifically, Fig. 32 shows the coding gain versus estimated complexity for the $\mathrm{CC}(2,1, K)$, TC $(2,1,4)$, TBCH$(32,26)$, and TTCM-8PSK schemes, where the associated parameters are shown in Tables 3-6. All simulation results were obtained upon employing the space-time code $\mathbf{G}_{2}$ using one receiver over uncorrelated Rayleigh fading channels at an effective throughput of 2 BPS. For the turbo schemes TC $(2,1,4)$, TBCH $(32,26)$, and TTCM-8PSK, the increased estimated complexity is achieved by increasing the number of iterations from one to ten. However, CCs are decoded noniteratively. Therefore, in Fig. 32, we vary the constraint length $K$ of the CCs from three to ten, which results in

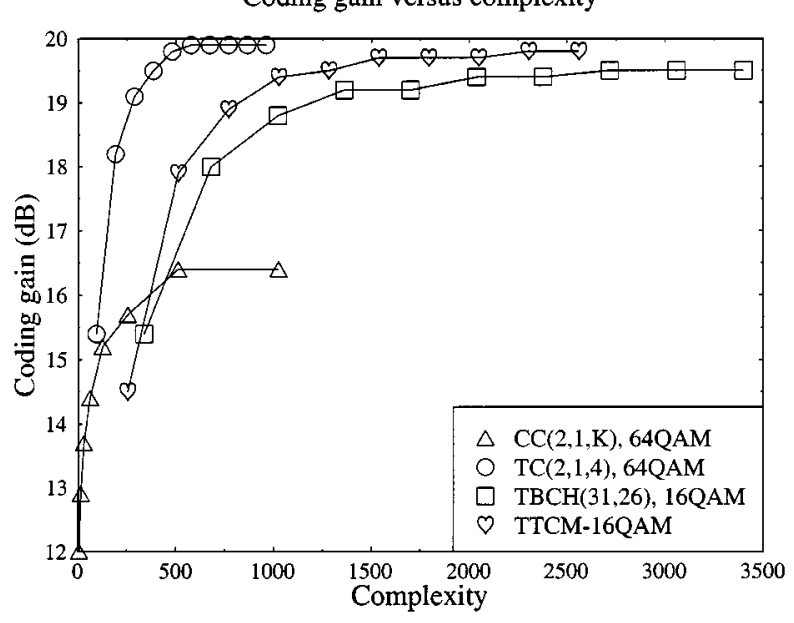

Fig. 33. Coding gain versus estimated complexity for the $\mathrm{CC}(2,1, K)$, TC $(2,1,4)$, TBCH$(31,26)$, and TTCM-16QAM schemes, where the coding parameters are shown in Tables 3-6. All simulation results were obtained upon employing space-time code $\mathbf{G}_{2}$ using one receiver over uncorrelated Rayleigh fading channels at an effective throughput of 3 BPS.

increased estimated complexity. The generator polynomials of the $\mathrm{CC}(2,1, K)$ codec, where $K=3 \cdots 10$, are given in [100] and they define the corresponding maximum-minimum free distance of the codes. From Fig. 32, we can see that there is a steep increase in the coding gain achieved by the $\mathrm{TC}(2,1,4)$ code, as the estimated complexity is increased. Moreover, the $\mathrm{TC}(2,1,4)$ scheme asymptotically achieves a maximum coding gain of approximately $20 \mathrm{~dB}$. At a low estimated complexity of approximately 200, the $\mathrm{TC}(2,1,4)$ code attains a coding gain of approximately 18 $\mathrm{dB}$, which exceeds that of the other channel codes studied. The $\operatorname{TBCH}(32,26)$ arrangement is the least attractive one, since a major complexity is incurred when aiming for a high coding gain.

In contrast to the 2 BPS schemes of Fig. 32, Fig. 33 shows the corresponding coding gain versus estimated complexity curves for the $\mathrm{CC}(2,1, K), \mathrm{TC}(2,1,4), \mathrm{TBCH}(31,26)$, and TTCM-16QAM 3 BPS arrangements, where the coding parameters are shown in Tables 3-6. Again, all simulation results were obtained upon employing the space-time code $\mathbf{G}_{2}$ using one receiver over uncorrelated Rayleigh fading channels at an effective throughput of 3 BPS. As before, the increased estimated complexity of the turbo schemes is incurred by increasing the number of iterations from one to ten. For the CCs, the constraint length $K$ is varied from three to ten. Similarly to Fig. 32, the TC $(2,1,4)$ scheme achieves a considerable coding gain at a relatively low estimated complexity. For example, in order to achieve a coding gain of $18 \mathrm{~dB}$, the TTCM and $\operatorname{TBCH}(31,26)$ arrangements would require approximately three and four times higher computational power compared to the $\mathrm{TC}(2,1,4)$ code.

From Figs. 32 and 33, we can clearly see that turbo codes are the most attractive one of all the channel codes studied in conjunction with the space-time code $\mathbf{G}_{2}$, offering an impressive coding gain at a moderate estimated decoding complexity. In Fig. 34, we show the $E_{b} / N_{0}$ value required for 


\section{$\mathrm{E}_{\mathrm{b}} / \mathrm{N}_{0}$ versus BPS}

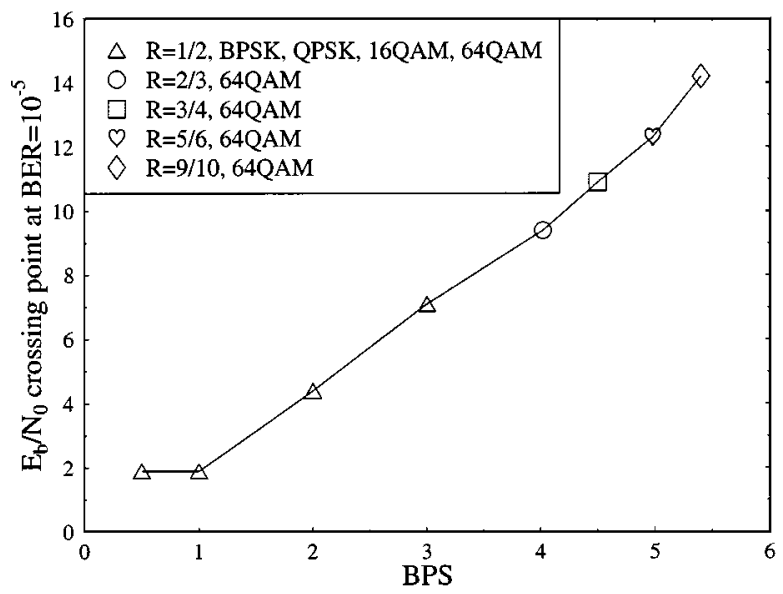

Fig. 34. $E_{b} / N_{0}$ value required for maintaining $\mathrm{BER}=10^{-5}$ versus the effective throughput BPS for the STB code $\mathbf{G}_{2}$ concatenated with the $\mathrm{TC}(2,1,4)$ code, where the coding parameters are shown in Tables 3, 5, and 6. All simulation results were obtained upon employing space-time code $\mathbf{G}_{2}$ using one receiver over uncorrelated Rayleigh fading channels.

maintaining BER $=10^{-5}$ versus the effective throughput BPS for the STB code $\mathbf{G}_{2}$ concatenated with the TC $(2,1,4)$ code where the coding parameters are shown in Tables 3, 5, and 6. All simulation results were obtained upon employing space-time code $\mathbf{G}_{2}$ using one receiver over uncorrelated Rayleigh fading channels. Half-rate $\mathrm{TC}(2,1,4)$ code was employed for BPS up to three. Then, TC $(2,1,4)$ code with various rates was employed with 64QAM in order to achieve increasing effective throughput BPS. It can be seen from the figure that the $E_{b} / N_{0}$ value required for maintaining BER $=10^{-5}$ increases linearly as the effective throughput BPS increases.

\section{E. Comparative Study of Concatenated Turbo-Coded and STB-Coded as Well as STT-Coded OFDM for Transmission Over Wide-Band Channels}

In Section VI-C, we found that for transmission over uncorrelated Rayleigh channels the half-rate TC was the highest gain channel coder when concatenated with the space-time code $\mathbf{G}_{2}$. In this section, we further our comparative investigations by comparing the performance of the TC-coded STB code $\mathbf{G}_{2}$ with STT codes in the context of OFDM [101], when transmitting over wide-band channels. Conventionally, RS codes are also employed in conjunction with STT codes for improving the performance of the system [98], which will be also invoked in our study. The employment of OFDM facilitated space-time-coded transmissions over wide-band channels, since OFDM maps the high-rate serial bitstream to a high number of low-rate subchannels transmitted in parallel. Hence, each subchannel becomes nondispersive.

1) System Overview: Fig. 35 shows the schematic of the system. At the transmitter, the information source generates random information data bits. The information bits are then encoded by $\mathrm{TC}(2,1,3)$ codes, RS codes, or left uncoded. Only the TC(2,1,3)-coded bits are channel interleaved and the output bits are then passed to the STT or

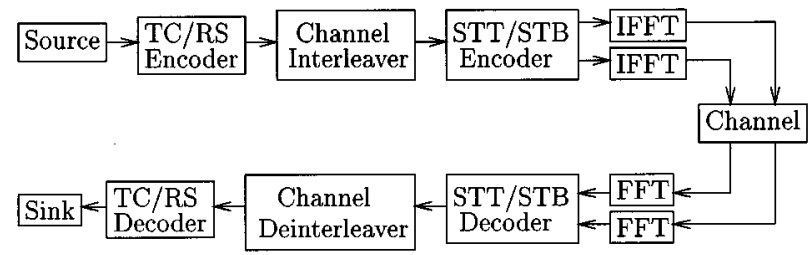

Fig. 35. System overview of channel-coded STB and trellis codes.

Table 8

Parameters of the STT Codes [13]

\begin{tabular}{c|c|c|c|c}
\hline $\begin{array}{c}\text { Modulation } \\
\text { scheme }\end{array}$ & BPS & $\begin{array}{c}\text { Decoding } \\
\text { algorithm }\end{array}$ & $\begin{array}{c}\text { No. of } \\
\text { trellis states }\end{array}$ & $\begin{array}{c}\text { No. of } \\
\text { termination symbols }\end{array}$ \\
\hline 4PSK & 2 & VA & 4 & 1 \\
\cline { 4 - 5 } & & & 8 & 2 \\
\cline { 3 - 5 } & & & $\mathbf{1 6}$ & 2 \\
\cline { 4 - 5 } & & & 32 & 3 \\
\hline 8PSK & 3 & VA & $\mathbf{1 6}$ & 2 \\
\hline
\end{tabular}

STB encoder [98] of Fig. 35. We will investigate the STT codes proposed in [13]. The modulation schemes employed were 4-level phase shift keying (4PSK) as well as 8PSK. On the other hand, from the family of STB codes, only Alamouti's $\mathbf{G}_{2}$ code is employed in this system, since again we have shown in Sections VI-A and VI-C that the best performance is achieved by concatenating the STB code $\mathbf{G}_{2}$ with $\mathrm{TC}(2,1,3)$ codes. In order to achieve high throughputs, 16QAM can be employed [101]. Gray mapping of the bits to symbols was applied and this resulted in different integrity protection classes in higher order modulation schemes [101]. The output of the space-time encoder was then OFDM [101] modulated with the aid of the inverse fast fourier transform blocks of Fig. 35 and transmitted by the corresponding antenna. The number of transmit antennas was fixed to two, while the number of receive antennas constituted a design parameter. Dispersive wide-band channels were used and the associated channels' profiles will be discussed at a later stage.

At the receiver, the signal of each receive antenna is OFDM demodulated. The demodulated signals of the receiver antennas are then fed to the STT or STB decoder. The space-time decoders apply the Log-MAP [75], [102] decoding algorithms for providing soft outputs for the channel decoders. If no channel codecs are employed in the system, the space-time decoders apply the VA [13], which gives a slightly lower performance compared to the MAP decoder, but at a lower complexity. The decoded bits are finally passed to the sink, as seen in Fig. 35, for the calculation of the BER or frame error ratio (FER).

2) Space-Time and Channel Codec Parameters: In Fig. 35, we have given an overview of the proposed system. In this section, we present the parameters of the STT codes and the channel codecs employed in the proposed system. We will employ the set of various STT codes proposed in [13]. The associated STT coding parameters are summarized in Table 8. 
Table 9

Additional System Parameters Associated With the TC $(2,1,3)$ Code

\begin{tabular}{l|c|c|c|c|c}
\hline & $\begin{array}{c}\text { Code } \\
\text { Rate, } R\end{array}$ & $\begin{array}{c}\text { Modulation } \\
\text { Mode }\end{array}$ & BPS & $\begin{array}{c}\text { Random turbo } \\
\text { interleaver depth }\end{array}$ & $\begin{array}{c}\text { Random separation } \\
\text { interleaver depth }\end{array}$ \\
\hline $\mathrm{TC}(2,1,3)$ & 0.50 & 16QAM & 2 & 256 & 512 \\
\hline
\end{tabular}

Again, in this system, we will concentrate on using the simple half-rate $\mathrm{TC}(2,1,3)$ code. Its associated parameters are shown in Table 3. As seen in Table 9, in conjunction with the half-rate $\mathrm{TC}(2,1,3)$ code, a higher order bit/symbol modulation scheme namely 16QAM was chosen, so that the effective 2 BPS throughput of the system remained the same as that of the system employing the 2-BPS STT codes without additional channel coding. It is widely recognized that the performance of TC codes improves upon increasing the turbo interleaver size and near-Shannonian performance can be achieved using large interleaver sizes exceeding 10000 bits. However, this performance gain is achieved at the cost of high latency, which is impractical in a delay-sensitive real-time system. On the other hand, STT codes offer impressive coding gains [13] at low latency. The decoding of the STT codes is carried out on a transmission burst-by-burst basis. In order to make a fair comparison between the systems investigated, the turbo interleaver size was chosen such that all the coded bits of a specific interleaved block were hosted by one transmission burst. This enables burst-by-burst turbo decoding at the receiver.

In Table 9, we summarized the interleaver sizes used in the proposed system. Again, the random-separation-based channel interleaver of Section VI-B was used. The mapping of the data bits and parity bits into different protection classes of the higher order modulation scheme [101] was carried out such that the best possible performance was attained. Following the rationale of [98], RS codes [101] were employed in conjunction with the STT codes. Hard-decision RS decoding was utilized and the coding parameters of the RS codes employed are summarized in Table 10.

3) STB Codes Versus STT Codes: In this section, we provide simulation results for space-time-coded OFDM [101] schemes using 128 subcarriers. Each OFDM symbol has a symbol duration of $160 \mu$ s and a cyclic prefix of $40 \mu$ s duration. In these simulations, the Jakes model was adopted for modeling the fading channels. We assume an equal-power two-path CIR, where the CIR taps are separated by a delay spread of $5 \mu \mathrm{s}$. The maximum Doppler frequency was $200 \mathrm{~Hz}$. All multipath components undergo independent Rayleigh fading and the receiver has a perfect knowledge of the CIR.

In Fig. 36, we show our FER performance comparison between the various 2 BPS effective throughput schemes, namely, the 4PSK STT codes employing no channel coding and the STB code $\mathbf{G}_{2}$ concatenated with the $\mathrm{TC}(2,1,3)$ code and the 16-state 8PSK STT code concatenated with the $\operatorname{RS}(63,42)$ code constructed over $\mathrm{GF}\left(2^{6}\right)$ using one receiver and the 128-subcarrier OFDM modem. Again,
Table 10

Coding Parameters of the RS Codes Employed

\begin{tabular}{l|c|c|c}
\hline Code & Galois Field & Code rate & Correctable symbol errors \\
\hline $\operatorname{RS}(63,42)$ & $2^{6}$ & 0.67 & 10 \\
\hline
\end{tabular}

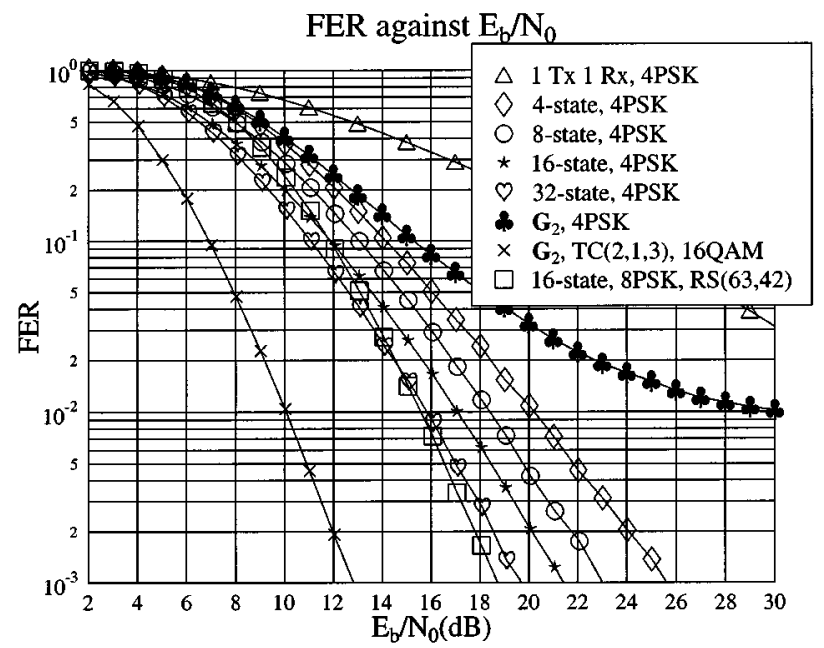

Fig. 36. FER performance comparison between various 4PSK STT codes and the STB code $\mathbf{G}_{2}$ concatenated with the TC $(2,1,3)$ code and the 16-state 8PSK STT code concatenated with the RS $(63,42)$ code over $\mathrm{GF}\left(2^{6}\right)$ using one receiver and the 128-subcarrier OFDM modem over a channel having a CIR characterized by two equal-power rays separated by a delay spread of $5 \mu \mathrm{s}$. Maximum Doppler frequency was $200 \mathrm{~Hz}$. Effective throughput was 2 BPS and the coding parameters are shown in Tables 8, 3, 9, and 10.

since the $\mathrm{TC}(2,1,3)$ code is a half-rate code and hence 16QAM was employed for absorbing its parity bits, in order to support the same 2-BPS effective throughput as the 4PSK STT codes using no channel codes. Similarly, the $\operatorname{RS}(63,42)$ code has a coding rate of about two-thirds and, hence, the 16-state 8PSK STT code was employed for maintaining a similar effective throughput of 2 BPS. We can clearly see that at FER $=10^{-3}$, the performance of the $\mathrm{TC}(2,1,3) / \mathrm{G}_{2}$ concatenated scheme is at least $7 \mathrm{~dB}$ better than that of the 4PSK STT codes. The concatenation of the 16-state 8PSK STT code and the RS $(63,42)$ code improves the performance and outperforms the 4PSK STT codes. However, its performance is still inferior to that of the $\mathrm{TC}(2,1,3) / \mathbf{G}_{2}$ scheme.

Here, we will address the implementational complexity issues of the proposed system. We will, however, focus mainly on the relative complexity of the proposed systems rather than attempting to quantify their exact complexity. In order to simplify our comparative study, several assumptions were 


\section{Coding gain versus complexity}

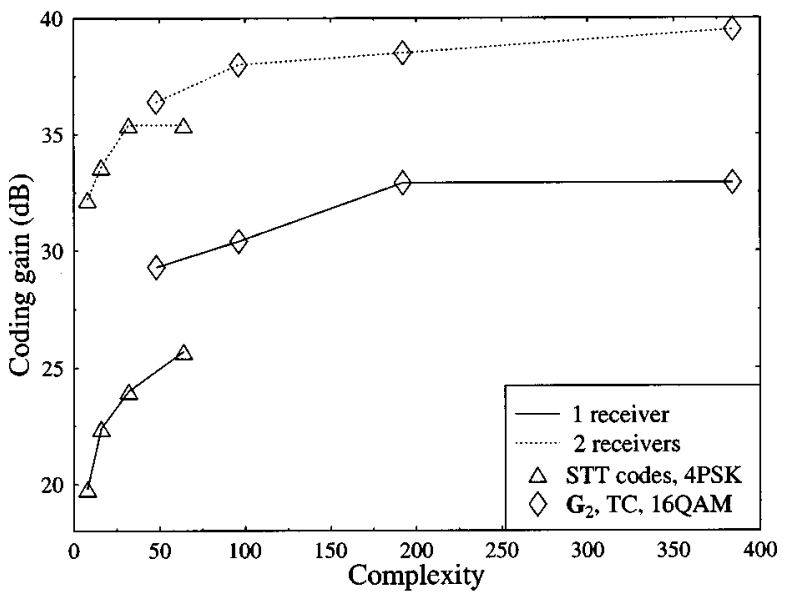

Fig. 37. Coding gain versus estimated complexity for the various 4PSK STT codes and the STB code $\mathbf{G}_{2}$ concatenated with the $\mathrm{TC}(2,1,3)$ code using one as well as two receivers and the 128-subcarrier OFDM modem over a channel having a CIR characterized by two equal-power rays separated by a delay spread of $5 \mu \mathrm{s}$. Maximum Doppler frequency was $200 \mathrm{~Hz}$. Effective throughput was 2 BPS and the coding parameters are shown in Tables 3, 8, and 9 .

stipulated. In our simplified approach, the estimated complexity of the system is deemed to depend only on that of the STT decoder and turbo decoder. In other words, the complexity associated with the modulator, demodulator, STB encoder and decoder, as well as that of the STT encoder and turbo encoder are assumed to be insignificant compared to the complexity of STT decoder and turbo decoder.

The estimated complexity of the turbo decoder was given in (37). On the other hand, from the state diagrams shown in [13], we can see that the number of trellis transitions leaving each trellis state is equivalent to $2^{\mathrm{BPS}}$, where again, BPS denotes the number of transmitted bits per modulation symbol. Since the number of information bits is equal to BPS, we can approximate the complexity of the STT decoder as

$$
\operatorname{comp}\{\mathrm{STT}\}=\frac{2^{\mathrm{BPS}} \times \text { No. of States }}{\text { BPS }} .
$$

By employing (37) and (51), we compare the performance of the proposed schemes by considering their approximate complexity. Our performance comparison of the various schemes in Fig. 37 was carried out on the basis of the coding gain defined as the $E_{b} / N_{0}$ difference, expressed in decibels $(\mathrm{dB})$, at FER $=10^{-3}$ between the proposed schemes and the uncoded single-transmitter single-receiver system having the same effective throughput of 2 BPS. Specifically, in Fig. 37, we show our coding gain versus estimated complexity comparison for the various 4PSK STT codes and for the STB code $\mathbf{G}_{2}$ concatenated with the $\mathrm{TC}(2,1,3)$ code using one as well as two receivers. Again, the 128-subcarrier OFDM modem was transmitting over the channel having a CIR of two equal-power rays separated by a delay spread of $5 \mu \mathrm{s}$ and a maximum Doppler frequency of $200 \mathrm{~Hz}$. The estimated complexity of the STT codes was increased by increasing the number of trellis states. By contrast, the estimated complexity of the $\mathrm{TC}(2,1,3)$ code was increased by increasing the number of turbo iterations. Therefore, the coding gain of the concatenated $\mathbf{G}_{2} / \mathrm{TC}(2,1,3)$ scheme using one, two, four, and eight iterations is shown in Fig. 37. It can be seen that the concatenated scheme outperforms the STT codes using no channel coding, even though the number of turbo iterations was only one. Moreover, the improvement in coding gain was obtained at an estimated complexity comparable to that of the 32-state 4PSK STT code using no channel coding. From Fig. 37, we can also see that the performance gain of the concatenated $\mathbf{G}_{2} / \mathrm{TC}(2,1,3)$ channel-coded scheme over the STT codes becomes lower when the number of receivers is increased to two.

\section{CONCLUSION}

State-of-the-art transmission schemes based on multiple transmitters and receivers were reviewed in Section I, while the history of channel coding was summarized in Section II. These discussions were followed by a rudimentary introduction to the MRC [23] technique, using a simple example in Section III-A. STB codes were introduced in Section IV, employing the unity-rate space-time code $\mathbf{G}_{2}$. In Sections IV-A1 and IV-A2, two examples of employing the space-time code $\mathbf{G}_{2}$ were provided using one and two receivers, respectively. The transmission matrix of a range of different-rate space-time codes, namely, that of the codes $\mathbf{G}_{3}, \mathbf{G}_{4}, \mathbf{H}_{3}$, and $\mathbf{H}_{4}$ of Table 2 were also given.

In Section V, we proposed a system that consists of the concatenation of the above-mentioned STB codes and a range of different channel codes. The channel coding schemes investigated were CCs, TC codes, TBCH codes, TCM, and TTCM. The estimated complexity and memory requirement of the channel decoders were compared in Section V-C.

Finally, we presented our simulation results in Section VI, which were divided into four categories. In Section VI-A, we first compared the performance results of the space-time codes $\mathbf{G}_{2}, \mathbf{G}_{3}, \mathbf{G}_{4}, \mathbf{H}_{3}$, and $\mathbf{H}_{4}$ without using channel codecs. It was found that as we increased the effective throughput of the system, the performance of the half-rate space-time codes $\mathbf{G}_{3}$ and $\mathbf{G}_{4}$ degraded in comparison to that of the unity rate space-time code $\mathbf{G}_{2}$. This was because higher modulation schemes had to be employed in conjunction with the half-rate space-time codes $\mathbf{G}_{3}$ and $\mathbf{G}_{4}$ in order to maintain the same effective throughput; these are more prone to errors and, hence, the performance of the system degrades. On the other hand, on the basis of maintaining the same diversity gain and same effective throughput, we found that the performance of the space-time codes $\mathbf{H}_{3}$ and $\mathbf{H}_{4}$ was better than that of the space-time codes $\mathbf{G}_{3}$ and $\mathbf{G}_{4}$, respectively. Since the space-time code $\mathbf{G}_{2}$ has a code rate of unity, we were able to concatenate it with half-rate TC codes, while maintaining the same effective throughput, as the half-rate space-time code without channel coding. Hence, for the same effective throughput, the unity-rate $\mathbf{G}_{2}$ space-time-coded and half-rate channel-coded scheme provided substantial performance improvement over the three-quarter rate space-time code $\mathbf{H}_{4}$ and half-rate space-time code $\mathbf{G}_{4}$, which were unable to benefit from 
channel coding. We concluded that the reduction in coding rate was best invested in turbo channel codes, rather than STB codes. Therefore, all channel codes studied were concatenated with the unity-rate space-time code $\mathbf{G}_{2}$ only.

In the second category of our investigations in Section VI-B, we studied the effect of the binary channel codes' data and parity bits mapped into different protection classes of multilevel modulation schemes. It was found that TC codes having different constraint lengths $K$ require different mapping methods. By contrast, in the TBCH codes studied mapping of the parity bits to the higher integrity protection class of a multilevel modulation scheme yielded a better performance. The so-called random-separation-based interleaver was proposed in order to improve the performance of the system.

The third set of results compared the performances of all proposed channel codes in conjunction with the space-time code $\mathbf{G}_{2}$. In order to avoid confusion, we only selected one channel code from each group of channel codes in Table 3. Specifically, only half-rate TC codes were studied, as they gave better coding gain performance compared to other TC codes having lower and higher rates. It was found that the performance of the half-rate TC codes was better than that of the CC, TBCH, TCM, and TTCM codes. Then, we compared the performance of high-rate TC codes with high-rate TBCH codes in conjunction with 64QAM. It was found that the $\mathrm{TBCH}$ codes provided a slight performance improvement over high-rate TC codes, but at the cost of high complexity. The discussions of the section were concluded by comparing the concatenated $\mathbf{G}_{2}$ space-time-coded channel codes upon taking their estimated complexity into consideration. The half-rate TC codes give the best coding gain at a moderate estimated complexity.

Finally, we probed further by comparing the performances of the TC-coded STB code $\mathbf{G}_{2}$ with STT codes. Again, we found that the TC-coded STB code $\mathbf{G}_{2}$ outperformed the STT codes considered at a comparable complexity. Finally, we conclude that the concatenation of the STB code $\mathbf{G}_{2}$ with TC codes provided the highest coding gain in the scenarios considered at a comparable complexity.

In conclusion, with the invention of turbo codes, the predictions of Shannon have been asymptotically approached. With the advent of space-time codes, the past 50 years of coding research has reached a state of maturity, where attractive coding schemes can be designed specifically for wireless channels. Hence, the challenge for coding researchers is now to achieve a performance close to the capacity of the wireless channel. No doubt that at the current pace of research, this will happen in a fraction of 50 years.

\section{REFERENCES}

[1] P. Chaudhury, "The 3GPP proposal for IMT-2000," IEEE Commun. Mag., vol. 37, pp. 72-81, Dec. 1999.

[2] G. Foschini, Jr. and M. Gans, "On limits of wireless communication in a fading environment when using multiple antennas," Wireless Pers. Commun., vol. 6, pp. 311-335, Mar. 1998.

[3] B. Glance and L. Greestein, "Frequency-selective fading effects in digital mobile radio with diversity combining," IEEE Trans. Commun., vol. COM-31, pp. 1085-1094, Sept. 1983.
[4] F. Adachi and K. Ohno, "BER performance of QDPSK with postdetection diversity reception in mobile radio channels," IEEE Trans. Veh. Technol., vol. 40, pp. 237-249, Feb. 1991.

[5] H. Zhou, R. Deng, and T. Tjhung, "Performance of combined diversity reception and convolutional coding for QDPSK land mobile radio," IEEE Trans. Veh. Technol., vol. 43, pp. 499-508, Aug. 1994.

[6] J. Winters, "Switched diversity with feedback for DPSK mobile radio systems," IEEE Trans. Inform. Theory, vol. IT-32, pp. 134-150, Feb. 1983

[7] G. Raleigh and J. Cioffi, "Spatio-temporal coding for wireless communications," in Proc. IEEE Global Telecommunications Conf., London, U.K., Nov. 1996, pp. 533-537.

[8] A. Wittneben, "Base station modulation diversity for digital SIMULCAST," in Proc. IEEE Vehicular Technolgy Conf., May 1993, pp. $505-511$.

[9] N. Seshadri and J. Winters, "Two signalling schemes for improving the error performance of frequency-division-duplex (FDD) transmission systems using transmitter antenna diversity," Int. J. Wireless Inform. Netw., vol. 1, pp. 49-60, Jan. 1994.

[10] J. Winters, "The diversity gain of transmit diversity in wireless systems with Rayleigh fading," IEEE Trans. Veh. Technol., vol. 47, pp. 119-123, Feb. 1998.

[11] T. Hattori and K. Hirade, "Multitransmitter simulcast digital signal transmission by using frequency offset strategy in land mobile radio-telephone system," IEEE Trans. Veh. Technol., vol. VT-27, pp. 231-238, Nov. 1978.

[12] A. Hiroike, F. Adachi, and N. Nakajima, "Combined effects of phase sweeping transmitter diversity and channel coding," IEEE Trans. Veh. Technol., vol. 41, pp. 170-176, May 1992.

[13] V. Tarokh, N. Seshadri, and A. Calderbank, "Space-time codes for high data rate wireless communication: Performance criterion and code construction," IEEE Trans. Inform. Theory, vol. 44, pp. 744-765, Mar. 1998.

[14] N. Seshadri, V. Tarokh, and A. Calderbank, "Space-time codes for high data rate wireless communications: Code construction," in Proc. IEEE Vehicular Technolgy Conf., Phoenix, AZ, 1997, pp. 637-641.

[15] V. Tarokh, N. Seshadri, and A. Calderbank, "Space-time codes for high data rate wireless communications: Performance criterion and code construction," in Proc. IEEE Int. Conf. Communications, Montreal, QB, Canada, 1997, pp. 299-303.

[16] V. Tarokh, A. Naguib, N. Seshadri, and A. Calderbank, "Space-time codes for high data rate wireless communications: Mismatch analysis," in Proc. IEEE Int. Conf. Communications, Montreal, QB, Canada, 1997, pp. 309-313.

[17] A. Naguib, V. Tarokh, N. Seshadri, and A. Calderbank, "A space-time coding modem for high-data-rate wireless communications," IEEE J. Select. Areas Commun., vol. 16, pp. 1459-1478, Oct. 1998.

[18] V. Tarokh, A. Naguib, N. Seshadri, and A. Calderbank, "Space-time codes for high data rate wireless communication: Performance criteria in the presence of channel estimation errors, mobility, and multiple paths," IEEE Trans. Commun., vol. 47, pp. 199-207, Feb. 1999.

[19] V. Tarokh, A. Naguib, N. Seshadri, and A. Calderbank, "Combined array processing and space-time coding," IEEE Trans. Inform. Theory, vol. 45, pp. 1121-1128, May 1999.

[20] G. Ungerboeck, "Trellis-coded modulation with redundant signal sets-Part I: Introduction,” IEEE Commun. Mag., vol. 25, pp. 5-11, Feb. 1987.

[21] — , "Trellis-coded modulation with redundant signal sets-Part II: State of the art," IEEE Commun. Mag., vol. 25, pp. 12-21, Feb. 1987.

[22] C. Schlegel, Trellis Coding. Piscataway, NJ: IEEE Press, 1997.

[23] S. Alamouti, "A simple transmit diversity technique for wireless communications," IEEE J. Select. Areas Commun., vol. 16, pp. 1451-1458, Oct. 1998.

[24] V. Tarokh, H. Jafarkhani, and A. Calderbank, "Space-time block codes from orthogonal designs," IEEE Trans. Inform. Theory, vol. 45, pp. 1456-1467, July 1999.

[25] - "Space-time block coding for wireless communications: Performance results," IEEE J. Select. Areas Commun., vol. 17, pp. 451-460, Mar. 1999.

[26] L. Hanzo, T. Liew, and B. L. Yeap, Turbo Coding, Turbo Equalization, and Space-Time Coding. New York: Wiley, 2002, to be published.

[27] C. Shannon, "A mathematical theory of communication," Bell Syst. Tech. J., vol. 27, pp. 379-656, July 1948. 
[28] R. Hamming, "Error detecting and error correcting codes," Bell Syst. Tech. J., vol. 29, pp. 147-160, 1950.

[29] P. Elias, "Coding for noisy channels," in IRE Nat. Conv. Rec., 1955, pp. 37-47.

[30] J. Wozencraft, "Sequential decoding for reliable communication," in IRE Nat. Conv. Rec., vol. 5, pt. 2, 1957, pp. 11-25.

[31] J. Wozencraft and B. Reiffen, Sequential Decoding. Cambridge, MA: MIT Press, 1961

[32] R. Fano, "A heuristic discussion of probabilistic coding," IEEE Trans. Inform. Theory, vol. IT-9, pp. 64-74, Apr. 1963.

[33] J. Massey, Threshold Decoding. Cambridge, MA: MIT Press, 1963.

[34] A. Viterbi, "Error bounds for convolutional codes and an asymptotically optimum decoding algorithm," IEEE Trans. Inform. Theory, vol. IT-13, pp. 260-269, Apr. 1967.

[35] G. Forney, "The Viterbi algorithm," Proc. IEEE, vol. 61, pp. 268-277, Mar. 1973.

[36] J. Heller and I. Jacobs, "Viterbi decoding for satellite and space communication," IEEE Trans. Commun. Technol., vol. COM-19, pp. 835-848, Oct. 1971.

[37] L. Bahl, J. Cocke, F. Jelinek, and J. Raviv, "Optimal decoding of linear codes for minimizing symbol error rate," IEEE Trans. Inform. Theory, vol. IT-20, pp. 284-287, Mar. 1974.

[38] C. Berrou, A. Glavieux, and P. Thitimajshima, "Near Shannon limit error-correcting coding and decoding: Turbo codes," in Proc. IEEE Int. Conf. Communications, Geneva, Switzerland, May 1993, pp. $1064-1070$.

[39] C. Berrou and A. Glavieux, "Near optimum error correcting coding and decoding: Turbo-codes," IEEE Trans. Commun., vol. 44, pp. 1261-1271, Oct. 1996.

[40] A. Hocquenghem, "Codes correcteurs d'erreurs," Chiffres (Paris), vol. 2, pp. 147-156, Sept. 1959.

[41] R. Bose and D. Ray-Chaudhuri, "On a class of error-correcting binary group codes," Information and Control, vol. 3, pp. 68-79, Mar. 1960.

[42] — - "Further results on error correcting binary group codes," Inform. Control, vol. 3, pp. 279-290, Sept. 1960.

[43] W. Peterson, "Encoding and error-correction procedures for the Bose-Chaudhuri-Hocquenghem codes," IRE Trans. Inform. Theory, vol. 6, pp. 459-470, Sept. 1960.

[44] J. Wolf, "Efficient maximum likelihood decoding of linear block codes using a trellis," IEEE Trans. Inform. Theory, vol. IT-24, pp. 76-80, Jan. 1978.

[45] B. Honary and G. Markarian, Trellis Decoding of Block Codes. Norwell, MA: Kluwer, 1997.

[46] S. Lin, T. Kasami, T. Fujiwara, and M. Fossorier, Trellises and Trellis-Based Decoding Algorithms for Linear Block Codes. Norwell, MA: Kluwer, 1998.

[47] G. Forney, "Coset codes-Part II: Binary lattices and related codes," IEEE Trans. Inform. Theory, vol. 34, pp. 1152-1187, Sept. 1988.

[48] H. Manoukian and B. Honary, "BCJR trellis construction for binary linear block codes," Proc. Inst. Electr. Eng. Commun., vol. 144, pp. 367-371, Dec. 1997

[49] B. Honary, G. Markarian, and P. Farrell, "Generalized array codes and their trellis structure," Electron. Lett., vol. 29, pp. 541-542, Mar. 1993.

[50] B. Honary and G. Markarian, "Low-complexity trellis decoding of Hamming codes," Electron. Lett., vol. 29, pp. 1114-1116, June 1993.

[51] B. Honary, G. Markarian, and M. Darnell, "Low-complexity trellis decoding of linear block codes," IEE Proc. Commun., vol. 142, pp. 201-209, Aug. 1995.

[52] T. Kasami, T. Takata, T. Fujiwara, and S. Lin, "On complexity of trellis structure of linear block codes," IEEE Trans. Inform. Theory, vol. 39, pp. 1057-1937, May 1993.

[53] _ - "On the optimum bit orders with respects to the state complexity of trellis diagrams for binary linear codes," IEEE Trans. Inform. Theory, vol. 39, pp. 242-245, Jan. 1993.

[54] D. Chase, "A class of algorithms for decoding block codes with channel measurement information," IEEE Trans. Inform. Theory, vol. IT-18, pp. 170-182, Jan. 1972.

[55] D. Gorenstein and N. Zierler, "A class of cyclic linear error-correcting codes in $p^{m}$ symbols," J. Soc. Ind. Appl. Math., vol. 9, pp. 107-214, June 1961

[56] I. Reed and G. Solomon, "Polynomial codes over certain finite fields," J. Soc. Ind. Appl. Math., vol. 8, pp. 300-304, June 1960.
[57] E. Berlekamp, "On decoding binary Bose-Chaudhuri-Hocquenghem codes," IEEE Trans. Inform. Theory, vol. IT-11, pp. 577-579, 1965.

[58] - Algebraic Coding Theory. New York: McGraw-Hill, 1968.

[59] J. Massey, "Step-by-step decoding of the Bose-Chaudhuri-Hocquenghem codes," IEEE Trans. Inform. Theory, vol. IT-11, pp. 580-585, 1965.

[60] — - "Shift-register synthesis and BCH decoding," IEEE Trans. Inform. Theory, vol. IT-15, pp. 122-127, 1969.

[61] M. Oh and P. Sweeney, "Bit-level soft-decision sequential decoding for Reed Solomon codes," in Proc. Workshop Coding and Cryptography, Paris, France, Jan. 1999.

[62] — "Low complexity soft-decision sequential decoding using hybrid permutation for rs codes," in Proc. Seventh IMA Conf. Cryptography and Coding, Cirencester, U.K., Dec. 1999.

[63] D. Burgess, S. Wesemeyer, and P. Sweeney, "Soft-decision decoding algorithms for RS codes," in Proc. Seventh IMA Conf. Cryptography and Coding, Cirencester, U.K., Dec. 1999.

[64] Consultative Committee for Space Data Systems, Blue Book: Recommendations for Space Data System Standards: Telemetry Channel Coding, May 1984.

[65] Digital video broadcasting (DVB); Framing structure, channel coding, and modulation for MVDS at 10GHz and above, ETSI 300 748 ed, Oct. 1996.

[66] F. Taylor, "Residue arithmetic: A tutorial with examples," IEEE Comput. Mag., vol. 17, pp. 50-62, May 1984.

[67] N. Szabo and R. Tanaka, Residue Arithmetic and Its Applications to Computer Technology. New York: McGraw-Hill, 1967.

[68] R. Watson and C. Hastings, "Self-checked computation using residue arithmetic," Proc. IEEE, vol. 54, pp. 1920-1931, Dec. 1966.

[69] H. Krishna, K. Lin, and J. Sun, "A coding theory approach to error control in redundant residue number systems-Part I: Theory and single error correction," IEEE Trans. Circuits Syst. II, vol. 39, pp. 8-17, Jan. 1992.

[70] J. Sun and H. Krishna, "A coding theory approach to error control in redundant residue number systems-Part II: Multiple error detection and correction," IEEE Trans. Circuits Syst. II, vol. 39, pp. 18-34, Jan. 1992.

[71] T. Liew, L. Yang, and L. Hanzo, "Soft-decision redundant residue number system based error correction coding," in Proc. Vehicular Technolgy Conf., Amsterdam, The Netherlands, Sept. 1999, pp. 2546-2550.

[72] R. Steele and L. Hanzo, Mobile Radio Communications. New York: Wiley, 1999.

[73] W. Koch and A. Baier, "Optimum and sub-optimum detection of coded data distributed by time-varying inter-symbol interference," in Proc. IEEE Global Telecommunications Conf., Dec. 1990, pp. 1679-1684.

[74] J. Erfanian, S. Pasupathy, and G. Gulak, "Reduced complexity symbol detectors with parallel structures for ISI channels," IEEE Trans. Commun., vol. 42, pp. 1661-1671, Feb./Mar./Apr. 1994.

[75] P. Robertson, E. Villebrun, and P. Hoeher, "A comparison of optimal and sub-optimal MAP decoding algorithms operating in the log domain," in Proc. Int. Conf. Communications, June 1995, pp. 1009-1013.

[76] J. Hagenauer and P. Hoeher, "A Viterbi algorithm with soft-decision outputs and its applications," in Proc. IEEE Global Telecommunications Conf., Dallas, TX, Nov. 1989, pp. 47.1.1-47.1.7.

[77] J. Hagenauer, "Source-controlled channel decoding," IEEE Trans. Commun., vol. 43, pp. 2449-2457, Sept. 1995.

[78] LAS. Goff, A. Glavieux, and C. Berrou, "Turbo-codes and high spectral efficiency modulation," in Proc. IEEE Int. Conf. Communications, New Orleans, May 1994, pp. 645-649.

[79] U. Wachsmann and J. Huber, "Power and bandwidth efficient digital communication using turbo codes in multilevel codes," Eur. Trans. Telecommun., vol. 6, pp. 557-567, Sept./Oct. 1995.

[80] P. Robertson and T. Wörz, "Bandwidth-efficient turbo trellis-coded modulation using punctured component codes," IEEE J. Select. Areas Commun., vol. 16, pp. 206-218, Feb. 1998.

[81] S. Benedetto and G. Montorsi, "Design of parallel concatenated convolutional codes," IEEE Trans. Commun., vol. 44, pp. 591-600, May 1996.

[82] — "Unveiling turbo codes: Some results on parallel concatenated coding schemes," IEEE Trans. Inform. Theory, vol. 42, pp. 409-428, Mar. 1996. 
[83] L. Perez, J. Seghers, and D. Costello, "A distance spectrum interpretation of turbo codes," IEEE Trans. Inform. Theory, vol. 42, pp. 1698-1709, Nov. 1996.

[84] J. Hagenauer, E. Offer, and L. Papke, "Iterative decoding of binary block and convolutional codes," IEEE Trans. Inform. Theory, vol. 42, pp. 429-445, Mar. 1996.

[85] R. Pyndiah, "Near-optimum decoding of product codes: Block turbo codes," IEEE Trans. Commun., vol. 46, pp. 1003-1010, Aug. 1998.

[86] H. Nickl, J. Hagenauer, and F. Burkert, "Approaching Shannon's capacity limit by $0.27 \mathrm{~dB}$ using simple Hamming codes," IEEE Commun. Lett., vol. 1, pp. 130-132, Sept. 1997.

[87] O. Acikel and W. Ryan, "Punctured turbo-codes for BPSK/QPSK channels," IEEE Trans. Commun., vol. 47, pp. 1315-1323, Sept. 1999.

[88] P. Jung and M. Nasshan, "Performance evaluation of turbo codes for short frame transmission systems," Electron. Lett., vol. 30, pp. 111-113, Jan. 1994.

[89] P. Jung, "Comparison of turbo-code decoders applied to short frame transmission systems," IEEE J. Select. Areas Commun., vol. 14, pp. 530-537, Apr. 1996.

[90] M. N. P. Jung and J. Blanz, "Application of turbo-codes to a CDMA mobile radio system using joint detection and antenna diversity," in Proc. IEEE Vehicular Technolgy Conf., 1994, pp. 770-774.

[91] A. Barbulescu and S. Pietrobon, "Interleaver design for turbo codes," Electron. Lett., vol. 30, pp. 2107-2108, Dec. 1994.

[92] B. Sklar, "A primer on turbo code concepts," IEEE Commun. Mag., pp. 94-102, Dec. 1997.

[93] G. Bauch, A. Naguib, and N. Seshadri, "MAP equalization of space-time coded signals over frequency selective channels," in Proc. Wireless Communications and Networking Conf., New Orleans, LA, Sept. 1999.

[94] G. Bauch and N. Al-Dhahir, "Reduced-complexity turbo equalization with multiple transmit and receive antennas over multipath fading channels," in Proc. Information Sciences and Systems, Princeton, NJ, Mar. 2000, pp. WP3 13-18.

[95] D. Agrawal, V. Tarokh, A. Naguib, and N. Seshadri, "Space-time coded OFDM for high data-rate wireless communication over wide-band channels," in Proc. IEEE Vehicular Technolgy Conf., Ottawa, ON, Canada, May 1998, pp. 2232-2236.

[96] Y. Li, N. Seshadri, and S. Ariyavisitakul, "Channel estimation for OFDM systems with transmitter diversity in mobile wireless channels," IEEE J. Select. Areas Commun., vol. 17, pp. 461-471, Mar. 1999.

[97] Y. Li, J. Chuang, and N. Sollenberger, "Transmitter diversity for OFDM systems and its impact on high-rate data wireless networks," IEEE J. Select. Areas Commun., vol. 17, pp. 1233-1243, July 1999.

[98] A. Naguib, N. Seshadri, and A. Calderbank, "Increasing data rate over wireless channels," IEEE Signal Processing Mag., vol. 17, pp. 76-92, May 2000.

[99] D. Brennan, "Linear diversity combining techniques," Proc. IRE, vol. 47, pp. 1075-1102, June 1959.

[100] J. Proakis, Digital Communications. New York: McGraw-Hill, 1995.

[101] L. Hanzo, W. Webb, W. Webb, W. Webb, and T. Keller, Single- and Multi-Carrier Quadrature Amplitude Modulation, 3rd ed. New York: Wiley, 2000.

[102] G. Bauch, "Concatenation of space-time block codes and TurboTCM," in Proc. IEEE Int. Conf. Communications, Vancouver, BC, Canada, June 1999, pp. 1202-1206.

[103] G. Forney, "Convolutional codes: I. Algebraic structure," IEEE Trans. Inform. Theory, vol. IT-16, pp. 720-738, Nov. 1970.

[104] - "Burst-correcting codes for the classic burst channel," IEEE Trans. Commun. Technol., vol. COM-19, pp. 772-781, Oct. 1971.

[105] B. Sklar, Digital Communications Fundamentals and Applications. Englewood Cliffs, NJ: Prentice-Hall, 1988.

[106] J. Hagenauer, "Rate-compatible punctured convolutional codes (RCPC codes) and their applications," IEEE Trans. Commun., pp. 389-400, Apr. 1988.

[107] S. Red, M. Oliphant, and M. Weber, An Introduction to GSM. Norwood, MA: Artech House, 1995.

[108] 3GPP. Multiplexing and channel coding (TDD). 3G TS 25.222. [Online]. Available: http://www.3gpp.org.

[109] T. Ojanperä and R. Prasad, Wideband CDMA for Third Generation Mobile Communications. Norwood, MA: Artech House, 1998.

[110] S. Al-Semari and T. Fuja, "I-Q TCM: Reliable communication over the Rayleigh fading channel close to the cutoff rate," IEEE Trans. Inform. Theory, vol. 43, pp. 250-262, Jan. 1997.
[111] W. Jakes, Microwave Mobile Communications. Piscataway, NJ: IEEE Press, 1993.

[112] R. Pyndiah, A. Glavieux, A. Picart, and S. Jacq, "Near optimum decoding of product codes," in Proc. IEEE Global Telecommunications Conf., San Francisco, CA, Nov. 1994, pp. 339-343.

[113] W. Peterson and E. Weldon, Jr., Error Correcting Codes, 2nd ed. Cambridge, MA: MIT Press, 1972.

[114] T. Kasami, Combinational Mathematics and Its Applications. Chapel Hill, NC: Univ. North Carolina Press, 1969.

[115] W. Peterson, Error Correcting Codes, 1st ed. Cambridge, MA: MIT Press, 1961.

[116] F. Macwilliams and N. Sloane, The Theory of Error Correcting Codes. Amsterdam, The Netherlands: North-Holland, 1978, vol. 16.

[117] I. Blake, Algebraic Coding Theory: History and Development. Stroudsburg, PA: Dowden, Hutchinson and Ross, 1973.

[118] G. Clark, Jr. and J. Cain, Error Correction Coding for Digital Communications. New York: Plenum Press, 1981.

[119] V. Pless, Introduction to the Theory of Error-Correcting Codes. New York: Wiley, 1982.

[120] R. Blahut, Theory and Practice of Error Control Codes. Reading, MA: Addison-Wesley, 1983.

[121] C. Shannon, Mathematical Theory of Communication. Urbana, IL: Univ. Illinois Press, 1963.

[122] C. Heegard and S. Wicker, Turbo Coding. Norwell, MA: Kluwer, 1999.

[123] M. Bossert, Channel Coding for Telecommunications. New York: Wiley, 1999.

[124] B. Vucetic and J. Yuan, Turbo Codes Principles and Applications. Norwell, MA: Kluwer, 2000.

[125] R. Lidl and H. Niederreiter, Finite Fields. Reading, MA: AddisonWesley, 1983.

[126] S. Lin and D. Costello, Jr., Error Control Coding: Fundamentals and Applications. Englewood Cliffs, NJ: Prentice-Hall, 1983.

[127] A. Michelson and A. Levesque, Error Control Techniques for Digital Communication. New York: Wiley, 1985.

[128] D. Hoffman, D. Leonard, C. Lindner, K. Phelps, C. Rodger, and J. Wall, Coding Theory. New York: Marcel Dekker, Inc., 1991.

[129] J. Huber, Trelliscodierung. Berlin, Germany: Springer-Verlag, 1992.

[130] J. Anderson and S. Mohan, Source and Channel Coding-An Algorithmic Approach. Norwell, MA: Kluwer, 1993.

[131] S. Wicker, Error Control Systems for Digital Communication and Storage. Englewood Cliffs, NJ: Prentice-Hall, 1994.

[132] P. Sweeney, Error Control Coding: An Introduction. Englewood Cliffs, NJ: Prentice-Hall, 1991.

T. H. Liew received the B.Eng. degree in electronics engineering and the Ph.D. degree in wireless communications from the University of Southampton, U.K., in 1997 and 2001, respectively.

He is currently continuing his research as a Postdoctoral Research Fellow with the University of Southampton. His current research interests are associated with coding and modulation for wireless channels, space-time coding, and adaptive transceivers and has published his research results widely.

Lajos Hanzo received the master's degree in electronics and the doctorate degree in telecommunications from the Technical University of Budapest, Hungary, in 1976 and 1983, respectively.

During his 25-year career in telecommunications, he has held various research and academic posts in Hungary, Germany, and the U.K. Since 1986, he has been with the Department of Electronics and Computer Science, University of Southampton, U.K., where he holds the Chair in Telecommunications. He has also been a Consultant to Multiple Access Communications Ltd., U.K. He is currently managing a research team, working on a range of research projects in the field of wireless multimedia communications under the auspices of the Engineering and Physical Sciences Research Council (EPSRC) U.K., the European IST Programme, and the Mobile Virtual Centre of Excellence (VCE). He has authored coauthored eight books on mobile radio communications and approximately 400 research papers, organized and chaired conference sessions, presented overview lectures, and was awarded a number of distinctions. He also provides a range of industrial training courses. 